\title{
G Protein-Coupled Receptors in Asthma Therapy: Pharmacology and Drug Action ${ }^{[\mathbb{1}}$
}

\author{
Stacy Gelhaus Wendell, Hao Fan, and Cheng Zhang
}

Department of Pharmacology and Chemical Biology, School of Medicine, University of Pittsburgh, Pittsburgh, Pennsylvania (S.G.W., C.Z.); Bioinformatics Institute, Agency for Science, Technology, and Research, Singapore (H.F.); and Department of Biological Sciences, National University of Singapore, and Center for Computational Biology, DUKE-NUS Medical School, Singapore (H.F.)

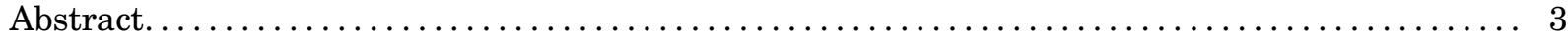

Significance Statement ............................................... 3

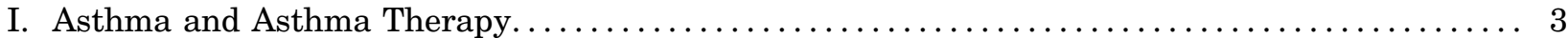

A. Epidemiology, Pathology, and Pathophysiology of Asthma $\ldots \ldots \ldots \ldots \ldots \ldots \ldots \ldots \ldots \ldots$

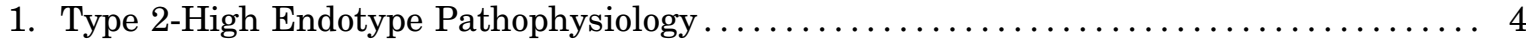

2. Type 2-Low Endotype Pathophysiology............................. 5

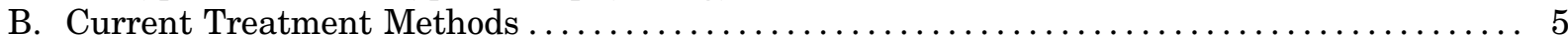

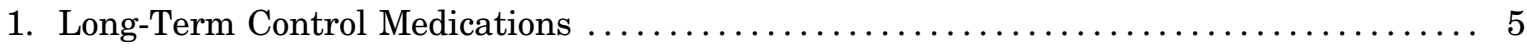

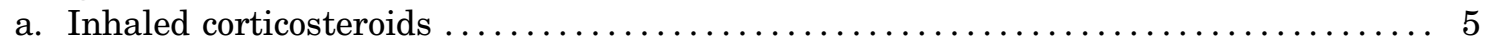

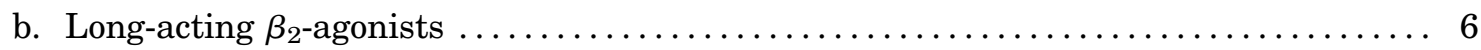

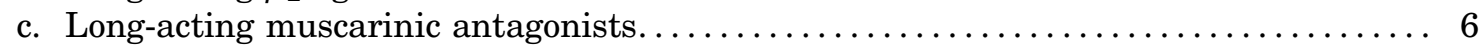

d. Leukotriene modifiers and leukotriene receptor antagonists $\ldots \ldots \ldots \ldots \ldots \ldots \ldots \ldots$

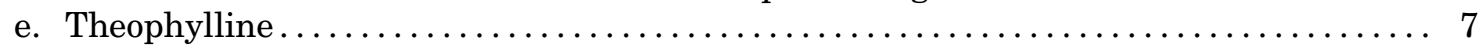

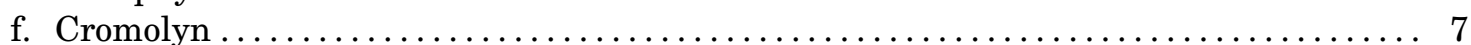

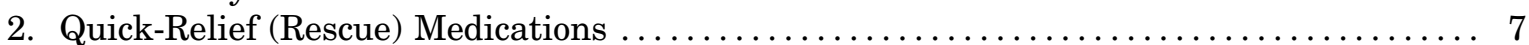

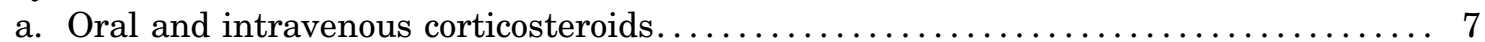

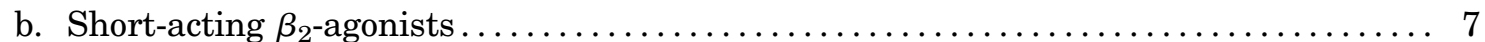

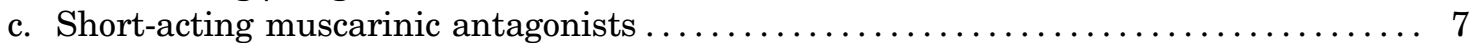

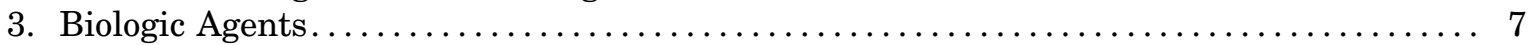

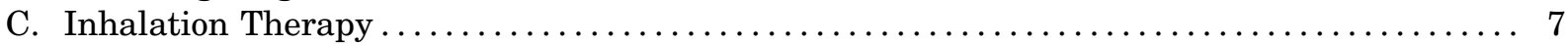

D. Preclinical Models of Asthma $\ldots \ldots \ldots \ldots \ldots \ldots \ldots \ldots \ldots \ldots \ldots \ldots \ldots \ldots \ldots \ldots \ldots \ldots$

E. G Protein-Coupled Receptors as Important Drug Targets in Asthma ................ 9

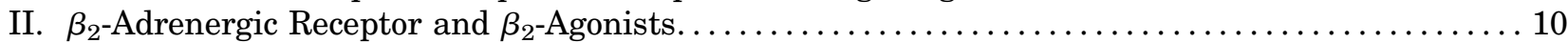

A. Pathophysiological Roles and Signaling of the $\beta_{2}$-Adrenergic Receptor in Asthma ........ 10

B. Currently Used $\beta_{2}$-Agonist Drugs and Their Pharmacological Properties ............. 11

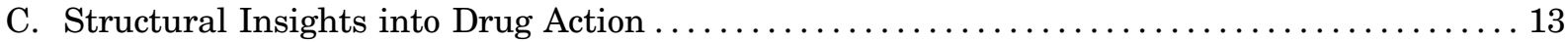

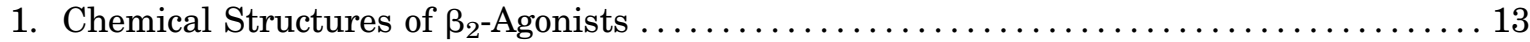

2. Structural Insights into the Pharmacological Properties of $\beta_{2}$-Agonists $\ldots \ldots \ldots \ldots \ldots 13$

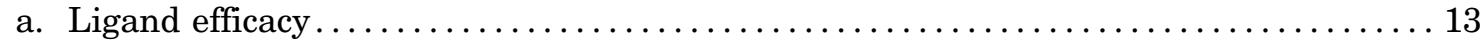

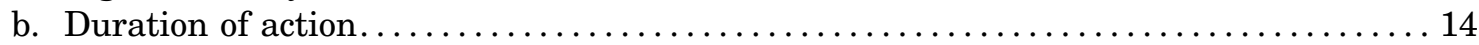

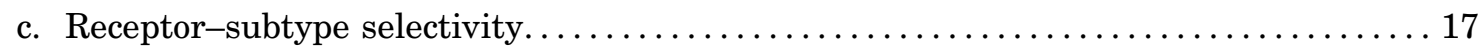

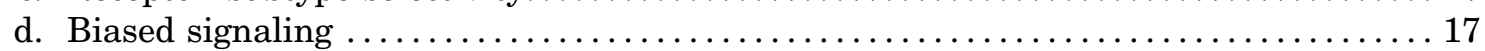

III. Muscarinic Acetylcholine Receptors and Muscarinic Antagonists.................... 17

A. Pathophysiological Roles and Signaling of Muscarinic Receptors in Asthma ........... 17

Address correspondence to: Dr. Hao Fan, Bioinformatics Institute, A*STAR, 30 Biopolis Street, Matrix \#07-01, Singapore 138671. E-mail: fanh@bii.a-star.edu.sg or Dr. Cheng Zhang, Department of Pharmacology and Chemical Biology, School of Medicine, University of Pittsburgh, 203 Lothrop Street, Pittsburgh, PA 15261. E-mail: chengzh@pitt.edu

This work was supported by the National Institutes of Health National Institute of General Medical Sciences [Grant 1R35GM128641 (to C.Z.)]; the National Institutes of Health National Institute of Allergy and Infectious Diseases [Grant R21AI122071 (to S.G.W.)]; and the Biomedical Research Council of A*STAR funding support (to H.F.).

https://doi.org/10.1124/pr.118.016899.

SThis article has supplemental material available at pharmrev.aspetjournals.org. 
B. Currently Used Muscarinic Antagonist Drugs and Their Pharmacological Properties ...... 18

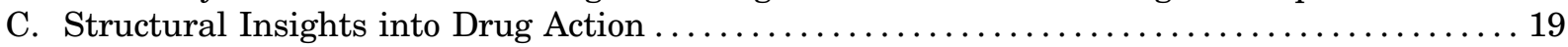

1. Chemical Structures of Clinically Used Muscarinic Antagonists . . . . . . . . . . . . 19

2. Structural Insights into the Pharmacological Properties of Clinically Used Muscarinic Antagonists............................................. 19

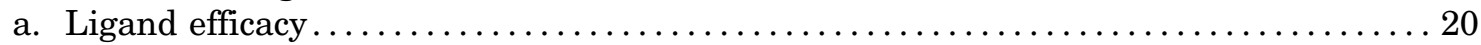

b. Receptor subtype selectivity in the orthosteric site $\ldots \ldots \ldots \ldots \ldots \ldots \ldots \ldots \ldots \ldots \ldots 22$

c. The structural basis for the action of allosteric modulators as potential novel

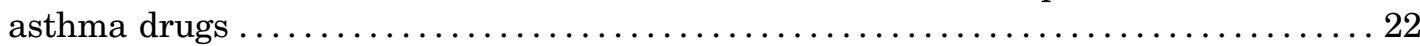

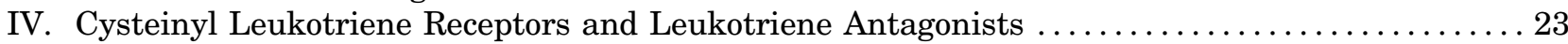

A. Pathophysiological Roles and Signaling of Cysteinyl Leukotriene Receptors in Asthma .... 23

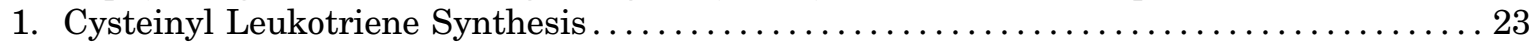

2. Pathophysiological Roles and Signaling of Cysteinyl Leukotriene Receptors.......... 23

B. Currently Used Leukotriene Receptor Antagonist Drugs and Their Pharmacological

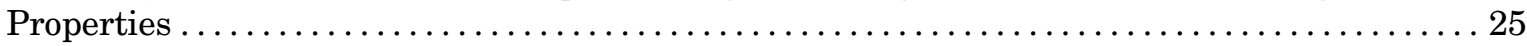

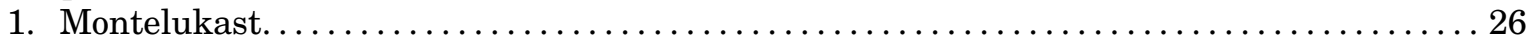

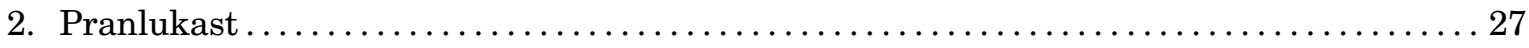

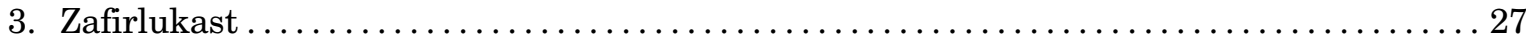

C. Structural Insights into Drug Action .................................. 27

V. Potential G Protein-Coupled Receptor-Targeting Drugs for Asthma in Late-Stage Clinical

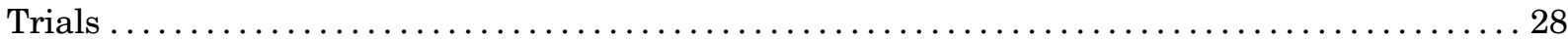

A. New G Protein-Coupled Receptor Targets for Developing Asthma Drugs............. 28

B. Prostaglandin $\mathrm{D}_{2}$ Receptor 2 and Its Antagonists ............................ 29

1. The Roles of Prostaglandin $\mathrm{D}_{2}$ Receptor 2 in Asthma......................... 29

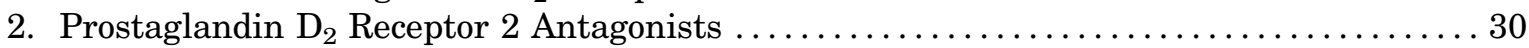

3. Structural Insights into the Action of Prostaglandin $\mathrm{D}_{2}$ Receptor 2 Antagonists....... 32

C. Potential Asthma Drugs Acting on Existing Asthma Targets...................... 32

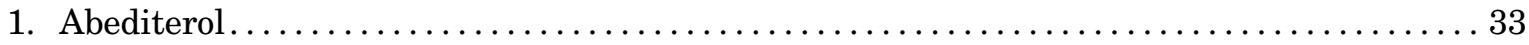

2. Bedoradrine .................................................. 33

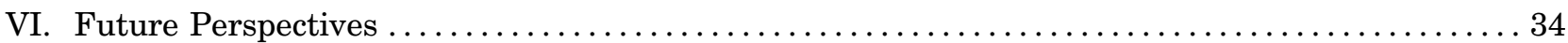

A. G Protein-Coupled Receptor Drugs in Early Stage of Development ................ 34

1. AZD8871 as a Dual-Action Ligand of Muscarinic Receptors and $\beta_{2}$-Adrenergic

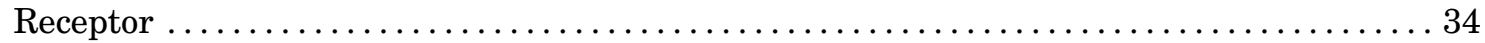

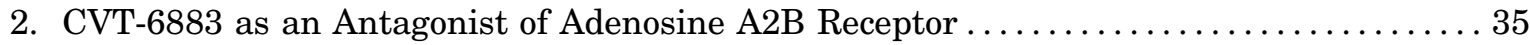

3. Toreforant as an Antagonist of Histamine H4 Receptor........................ 35

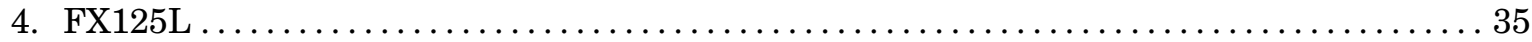

B. New Opportunities in G Protein-Coupled Receptor Drug Development for Asthma ........ 35

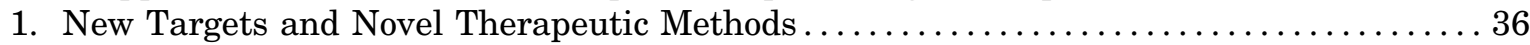

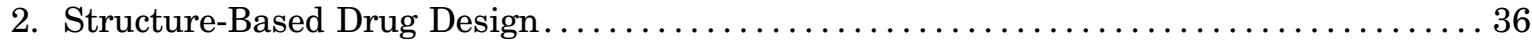

3. G Protein-Coupled Receptor Antibodies as Therapeutics ..................... 36

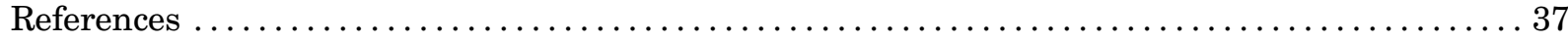

\footnotetext{
ABBREVIATIONS: 5-LOX, 5-lipoxygenase; 7TM, seven-transmembrane helix; AA, arachidonic acid; AHR, airway hyperresponsiveness; AIA, aspirin-induced asthma; ALX, lipoxin $\mathrm{A}_{4}$ receptor; ASM, airway smooth muscle; $\beta_{1} \mathrm{AR}, \beta_{1}$-adrenergic receptor; $\beta_{2} \mathrm{AR}, \beta_{2}$-adrenergic receptor; $\beta_{3} \mathrm{AR}, \beta_{3}$-adrenergic receptor; BLT, leukotriene receptor; COPD, chronic obstructive pulmonary disease; CysLT, cysteinyl leukotriene; CysLTR, CysLT receptor; DC, dendritic cell; DP1, $\mathrm{PGD}_{2}$ receptor 1; DP2, $\mathrm{PGD}_{2}$ receptor 2; ECL, extracellular loop; EIA, exerciseinduced asthma; FDA, Food and Drug Administration; FPR, formyl peptide receptor; $\mathrm{G}_{\mathrm{s}}, \alpha$ subunit of G $\mathrm{s}_{\mathrm{s}}$; GINA, Global Initiative for Asthma; GPCR, G protein-coupled receptor; H4R, histamine 4 receptor; HDM, house dust mite; ICS, inhaled corticosteroid; IL, interleukin; ILC2, group 2 innate lymphoid cell; LABA, long-acting $\beta_{2}$-agonist; LAMA, long-acting muscarinic antagonist; LTA 4 , leukotriene $\mathrm{A}_{4}$; LTB L $_{4}$ leukotriene $\mathrm{B}_{4} ; \mathrm{LTC}_{4}$, leukotriene $\mathrm{C}_{4} ; \mathrm{LTD}_{4}$, leukotriene $\mathrm{D}_{4} ; \mathrm{LTE}_{4}$, leukotriene $\mathrm{E}_{4} ; \mathrm{LTRA}$, leukotriene receptor antagonist; MABA, muscarinic antagonists $/ \beta_{2}$-agonist; MAPK, mitogen-activated protein kinase; MD, molecular dynamics; MDI, metered dose inhaler; MLCP, myosin-light chain phosphatase; MR, muscarinic receptor; NMS, N-methyl scopolamine; OVA, ovalbumin; PAM, positive allosteric modulator; PAR, protease-activated receptor; PDB, Protein Data Bank; PDE, phosphodiesterase; PG, prostaglandin; PKA, protein kinase A; PKC, protein kinase C; QNB, 3-quinuclidinyl benzilate; SABA, short-acting $\beta_{2}$-agonist; SAMA, short-acting muscarinic antagonist; SAR, structure and activity relationship; $\mathrm{T}_{\mathrm{H}}$, T helper; TSLP, thymic stromal lymphopoietin.
} 
Abstract - Asthma is a heterogeneous inflammatory disease of the airways that is associated with airway hyperresponsiveness and airflow limitation. Although asthma was once simply categorized as atopic or nonatopic, emerging analyses over the last few decades have revealed a variety of asthma endotypes that are attributed to numerous pathophysiological mechanisms. The classification of asthma by endotype is primarily routed in different profiles of airway inflammation that contribute to bronchoconstriction. Many asthma therapeutics target $G$ protein-coupled receptors (GPCRs), which either enhance bronchodilation or prevent bronchoconstriction. Short-acting and long-acting $\beta_{2}$-agonists are widely used bronchodilators that signal through the activation of the $\beta_{2}$-adrenergic receptor. Short-acting and long-acting antagonists of muscarinic acetylcholine receptors are used to reduce bronchoconstriction by blocking the action of acetylcholine. Leukotriene antagonists that block the signaling of cysteinyl leukotriene receptor 1 are used as an add-on therapy to reduce bronchoconstriction and inflammation induced by cysteinyl leukotrienes. A number of GPCR-targeting asthma drug candidates are also in different stages of development. Among them, antagonists of prostaglandin $\mathrm{D}_{2}$ receptor 2 have advanced into phase III clinical trials. Others, including antagonists of the adenosine $A_{2 B}$ receptor and the histamine $\mathrm{H} 4$ receptor, are in early stages of clinical investigation. In the past decade, significant research advancements in pharmacology, cell biology, structural biology, and molecular physiology have greatly deepened our understanding of the therapeutic roles of GPCRs in asthma and drug action on these GPCRs. This review summarizes our current understanding of GPCR signaling and pharmacology in the context of asthma treatment.

Significance Statement_-Although current treatment methods for asthma are effective for a majority of asthma patients, there are still a large number of patients with poorly controlled asthma who may experience asthma exacerbations. This review summarizes current asthma treatment methods and our understanding of signaling and pharmacology of $G$ proteincoupled receptors (GPCRs) in asthma therapy, and discusses controversies regarding the use of GPCR drugs and new opportunities in developing GPCRtargeting therapeutics for the treatment of asthma.

\section{Asthma and Asthma Therapy}

\section{A. Epidemiology, Pathology, and Pathophysiology of Asthma}

Asthma is among the most prevalent noncommunicable diseases worldwide. According to the reports from Global Asthma Network and Global Initiative for Asthma (GINA), asthma affects 1\%-18\% of the population in different countries with a global prevalence of over 300 million people. In the United States, the estimated prevalence of asthma in 2010 was $8.4 \%$ of the population (Loftus and Wise, 2016). Noticeably, this number has increased by $2.9 \%$ per year from 2001 to 2010. Asthma also affects about 6 million children between the ages of 0 and 17 in the United States, according to the Centers for Disease Control and Prevention. In fact, a recent review mentioned that asthma is the most common childhood noncommunicable disease (Chabra and Gupta, 2019). Because of the high prevalence and chronic nature, asthma brings a serious economic burden to the society. It was estimated that asthma was responsible for a total cost of $\$ 81.9$ billion dollars, including the medical costs and other indirect costs, in the United States for the year of 2013 (Nurmagambetov et al., 2018). Although the mortality rate of asthma has decreased significantly in the past decades, it is still among the top leading causes of years lived with disability and burden of disease (Global Asthma Network Report 2018).

Our understanding of asthma has changed significantly in the past two decades (Chung et al., 2014; Lambrecht and Hammad, 2015; Reddel et al., 2015; Pavord et al., 2018; Chabra and Gupta, 2019). Many studies have suggested a highly complex and heterogeneous nature of asthma. Therefore, asthma has been recognized and viewed more and more as a syndrome with a broad spectrum of distinct disease phenotypes and underlying pathophysiological mechanisms rather than one specific disorder (Eder et al., 2006; Wenzel, 2012; Wu et al., 2014; Pavord et al., 2018). The most recent GINA report defines asthma as a heterogeneous disease, which is mainly characterized by chronic airway inflammation. Common symptoms for asthma include cough, chest tightness, wheezing, and shortness of breath. Asthma is associated with variable airflow limitation, airway hyperresponsiveness (AHR), and sometimes airway remodeling, which are also the major characteristics of chronic obstructive pulmonary disease (COPD) (Hogg, 2004).

The definition of clinical phenotypes of asthma has attracted much attention in the past decade, which is still far from reaching a widely accepted consensus and remains controversial. Depending on various clinical, etiological, pathophysiological, or demographic characteristics, asthma can be clustered into different phenotypes (Haldar et al., 2008; Siroux and Garcia-Aymerich, 2011; Wenzel, 2012; Campo et al., 2013). Commonly recognized phenotypes include allergic and nonallergic asthma (or atopic and nonatopic asthma), late-onset persistent asthma, and obesity-driven asthma. However, a specific asthma phenotypic trait may have multiple underlying disease mechanisms linked to different mechanistic pathways (Green et al., 2002; Fahy, 2015). Therefore, it has been suggested that disease endotypes, which are defined by specific pathophysiological mechanisms, may provide a better framework for asthma research (Anderson, 2008; Perlikos et al., 2016; Papi et al., 2017; Licari et al., 2018). Biomarkers of each pathophysiological mechanism or mechanistic pathway could help to identify each endotype in the clinic, 
allowing for drugs or therapeutic methods that specifically target the underlying causative mechanism to be used for each endotype. The appropriate classification of asthma based on the phenotypes or endotypes is expected to provide a better understanding of asthma heterogeneity among patients so the therapeutic outcomes of specific treatment methods are more predictable. This will also facilitate the development of personalized and targeted therapies to fulfill currently unmet needs in the management and control of asthma, especially severe asthma.

Asthma has long been recognized as an inflammatory disease of the airways, particularly lower airways. Uncontrolled inflammation in the upper airways often leads to allergic rhinitis, which shares many common pathophysiological mechanisms with asthma (Locksley, 2010). Intensive research effort has been focused on the immune pathways and immunologic processes that lead to various asthma phenotypes in the past two decades. A major breakthrough in the asthma immunology was the identification of the critical roles of a subset of $\mathrm{CD}^{+} \mathrm{T}$ cells, the $\mathrm{T}$ helper $\left(\mathrm{T}_{\mathrm{H}}\right) 2$ cells, and the immune responses mediated by $\mathrm{T}_{\mathrm{H}} 2$ cells in allergic asthma (Grünig et al., 1998; Wills-Karp et al., 1998; Zhang et al., 1999). $\mathrm{T}_{\mathrm{H}} 2$ cells can produce type 2 cytokines, including interleukin (IL)-4, IL-5, and IL-13, which control and mediate type-2 immunity (Voehringer et al., 2006). Uncontrolled and chronic type 2 immunity in the airways leads to type 2 inflammation, which is the major driving force and the hallmark of allergic asthma (Locksley, 2010; Fahy, 2015). Recently, other types of adaptive immune cells, particularly the group 2 innate lymphoid cells (ILC2s), were also found to be important players in type 2 inflammation.

However, there is also a large body of evidence suggesting that many asthma patients, particularly patients with severe asthma, showed no or low characteristics of type 2 inflammation, indicating that additional pathophysiological mechanisms are involved (Wenzel et al., 1997; Jatakanon et al., 1999; Green et al., 2002; Woodruff et al., 2007, 2009). From these various studies, asthma has been roughly classified as two endotypes, type 2 inflammation-high (type 2-high) and type 2-low. It was estimated that most children and half of adult patients with asthma are type 2 inflammation-related, whereas for patients with severe asthma type 2, inflammation-related phenotypes only represent $37 \%$ of the population (Woodruff et al., 2009; Lambrecht and Hammad, 2015; Kuo et al., 2017). The type 2-low endotype represents an important and wellestablished severe asthma endotype, which is much less understood and much more diverse compared with the type 2-high endotype.

1. Type 2-High Endotype Pathophysiology. Type 2 immunity and type 2 inflammation are well studied and understood. They are strongly linked to allergic or atopic pathologies in diseases such as asthma and are characterized by the production of type 2 cytokines, IL-4, IL-5, and IL-13, and high levels of IgE antibodies (Voehringer et al., 2006). Type 2 inflammation involves many types of innate and adaptive immune cells, including $\mathrm{T}_{\mathrm{H}} 2$ cells, ILC2s, specific $\mathrm{B}$ cells that produce IgE antibodies, eosinophils, basophils, and mast cells. Multiple pathways mediated by those cells work together through complex and heterogeneous interplay to result in different asthma phenotypes displaying characteristics of type 2 inflammation.

It is well accepted that the type 2 inflammation starts from the events in the airway epithelium. Allergens and other stimuli interact with airway epithelial cells, which then release a series of cytokines such as thymic stromal lymphopoietin (TSLP) and IL-25 and IL-33. These cytokine regulators can then lead to the generation of type 2 cytokines through activating multiple immune cells to drive the onset of type 2 inflammation. Activated and primed dendritic cells (DCs) during this process promote the differentiation of naive $\mathrm{CD}^{+} \mathrm{T}$ cells into $\mathrm{T}_{\mathrm{H}} 2$ cells, which are the major cell type producing type 2 cytokines (Paul and Zhu, 2010). Two transcription factors, STAT6 and GATA3, are key to the activation of $\mathrm{T}_{\mathrm{H}} 2$ cells (Zheng and Flavell, 1997; Kuperman et al., 1998; Mathew et al., 2001; Hosokawa et al., 2013; Wan, 2014).

Another potent producer of type 2 cytokines are the recently discovered ILC2s (Fallon et al., 2006; Moro et al., 2010; Neill et al., 2010; Price et al., 2010; Walker et al., 2013). These innate lymphoid cells show some functional resemblance of $\mathrm{T}_{\mathrm{H}} 2$ cells. Although they do not have antigen receptors for $\mathrm{T}$ or $\mathrm{B}$ cells, they do express receptors for the cytokines produced by stimulated epithelial cells, TSLP, IL-25, and IL-33. Engagement of these cytokines with their receptors can lead the activation and recruitment of ILC2s, which in turn participate in the type 2 inflammation process by producing type 2 cytokines. Studies have demonstrated that they may produce even more IL-5 and IL-13 than $\mathrm{T}_{\mathrm{H}} 2$ cells under some circumstances (Chen et al., 2017). In addition, ILC2s may also contribute to the differentiation of naive $\mathrm{CD} 4^{+} \mathrm{T}$ cells into $\mathrm{T}_{\mathrm{H}} 2$ cells (Halim et al., 2014; Oliphant et al., 2014). The roles of ILC2s and $\mathrm{T}_{\mathrm{H}} 2$ cells in the production of type 2 cytokines and how they affect the functions of each other are still not fully understood. It is likely that ILC2s and $\mathrm{T}_{\mathrm{H}} 2$ cells may have different roles in different phenotypes of asthma. It is notable that ILC2s may show resistance to the steroid-induced suppression of type 2 cytokine production (Kabata et al., 2013). Because steroid drugs are the mainstream drugs for reducing inflammation in asthma, a better understanding of the function of ILC2s in certain types of asthma patients with ILC2s as the dominant players in type 2 inflammation is important for developing appropriate personalized treatment plans.

Type 2 cytokines are the central regulatory molecules of type 2 inflammation (Fahy, 2015; Wynn, 2015). IL-4 
can induce antibody class switching in B cells to result in IgE production. It also drives $\mathrm{T}$ cell differentiation to produce $\mathrm{T}_{\mathrm{H}} 2$ cells from naive $\mathrm{CD} 4^{+}$cells. IL-5 is a key regulator of the recruitment and development of eosinophils, another major cell type associated with type 2 inflammation. The activated eosinophils recruited to the airways can release a number of potent inflammatory mediators and disease-inducing molecules, including cytokines, cysteinyl leukotrienes (CysLT), and cytotoxic proteins. Eosinophilia in the airways causes eosinophilic asthma, which is an important type of asthma that is often severe. Other granulocytes such as mast cells and basophils also respond to IL-5. IL-13 is a primary effector that directly induces asthma symptoms. A well-characterized function of IL-13 is to drive the differentiation of airway epithelial cells into active goblet cells that produce mucins, a process called goblet cell metaplasia (Wills-Karp et al., 1998; Tanabe et al., 2008; Wynn, 2015). Overproduction of mucins leads to the formation of thick mucus and subsequent airway clogging. In addition, IL-13 can also induce AHR and contribute to airway remodeling and even pulmonary fibrosis (Rael and Lockey, 2011).

IgE antibodies produced by type 2 cytokine-induced $\mathrm{B}$ cells are another critical molecular controller of type 2-high asthma. In general, IgE antibodies are considered as the major characteristics of atopic diseases. In asthma, IgE antibodies can bind to the high-affinity IgE receptor FceRI that is highly expressed on mast cells, basophils, and DCs. Engagement and crosslinking of IgEs to their receptor lead to the migration and degranulation of mast cells and basophils and the activation of DCs, which then results in the release of potent inflammatory molecules, including histamine, inflammatory lipid mediators, and chemokines (Lambrecht and Hammad, 2015). These molecules are directly associated with airway hypersensitivity. Activated DCs can promote the differentiation of $\mathrm{CD} 4^{+} \mathrm{T}$ cells, which may be important for the development of chronic airway inflammation (Lambrecht et al., 1998). Basophils also have been shown to secrete IL-4, further amplifying type 2 inflammation (Nouri-Aria et al., 2001; Min et al., 2004; Kim et al., 2013).

2. Type 2-Low Endotype Pathophysiology. The variations of type 2-low asthma and the underlying pathophysiological mechanisms are far less understood compared with the type 2-high asthma. It is highly likely that the type 2-low endotype may actually be comprised of several distinct endotypes, all of which are associated with normal levels of type 2 cytokines (Fahy, 2015). Given the fact that less than half of severe asthma patients are associated with dominant type 2 inflammation, the pathophysiology of type 2-low asthma has attracted more and more research interest (Robinson et al., 2017). However, to date it is still enigmatic and there is no effective treatment method that specifically targets type 2-low asthma.
One characteristic found in many type 2-low asthma patients is the high level of neutrophils instead of eosinophils. Neutrophilic inflammation is usually associated with high levels of $\mathrm{T}_{\mathrm{H}} 1$ and $\mathrm{T}_{\mathrm{H}} 17$ cells (Shaw et al., 2007; McKinley et al., 2008). However, the mechanisms underlying the recruitment and activation of these cells and how they contribute to asthma symptoms are not clear. One study suggested the involvement of the intracellular NLRP3 (NACHT, LRR and PYD domains-containing protein 3) inflammasome in neutrophilic asthma (Simpson et al., 2014). Increased levels of cytokines produced by $\mathrm{T}_{\mathrm{H}} 17$ cells including IL-17A, IL-17F, and IL-22 have also been found in neutrophilic asthma. However, the functional roles of these cytokines are controversial. IL-17A has been suggested to contribute to airway remodeling (Bellini et al., 2012). Previous studies also suggested the involvement of other inflammatory mediators such as IL-8 and tumor necrosis factor- $\alpha$ in neutrophilic inflammation (Berry et al., 2006; Wood et al., 2012).

The mainstream anti-inflammatory steroid drugs are far less effective in reducing $\mathrm{T}_{\mathrm{H}} 17$ cytokines than reducing type 2 cytokines. Therefore, neutrophilic asthma driven by $\mathrm{T}_{\mathrm{H}} 17$ inflammatory mediators exhibits resistance to steroid therapies, which seems to be a common feature of type 2-low asthma. It is of paramount importance to identify different endotypes of type 2-low asthma and the underlying pathophysiological mechanisms to develop novel asthma therapies.

\section{B. Current Treatment Methods}

The pharmacological options for treatment and management of asthma include inhaled corticosteroids (ICSs), oral corticosteroids, long-acting $\beta_{2}$-agonists (LABAs), short-acting $\beta_{2}$-agonists (SABAs), long-acting muscarinic antagonists (LAMAs), short-acting muscarinic antagonists (SAMAs), leukotriene modifiers, theophylline, cromolyn, and immune-modulating antibody drugs, which are well summarized in the GINA Report 2018 and the Lancet paper by Pavord et al. (2018). Most of these medications fall into the following three main categories: 1) long-term control medications; 2) quickrelief medications; and 3) biologic agents (Table 1). Long-term control medications reduce airway inflammation that leads to chronic symptoms and include therapies such as ICSs, LABAs, LAMAs, leukotriene modifiers, theophylline, and cromolyn. Quick-relief medications quickly open constricted airways that limit breathing and include oral corticosteroids, SABAs, and SAMAs. In certain situations, biologic drugs are required, including the IgE antibody omalizumab and the anti-IL antibodies.

\section{Long-Term Control Medications.}

a. Inhaled corticosteroids. ICSs include beclomethasone dipropionate, budesonide, ciclesonide, flunisolide, fluticasone furoate, mometasone furoate, and fluticasone propionate. These medications reduce AHR and 
TABLE 1

Overview of current asthma drugs

\begin{tabular}{|c|c|c|c|c|c|}
\hline Drug & Category & Target & Mechanism of Action & Medical Use & Examples \\
\hline ICSs & $\begin{array}{l}\text { Long-term } \\
\text { control } \\
\text { medications }\end{array}$ & Glucocorticoid receptors & $\begin{array}{l}\text { Suppression of airway } \\
\text { inflammation }\end{array}$ & $\begin{array}{l}\text { Mild/Moderate } \\
\text { persistent asthma }\end{array}$ & $\begin{array}{l}\text { Budesonide, } \\
\text { beclomethasone, } \\
\text { fluticasone }\end{array}$ \\
\hline LABAs & $\begin{array}{l}\text { Long-term } \\
\text { control } \\
\text { medications }\end{array}$ & $\beta_{2} \mathrm{AR}$ & $\begin{array}{l}\text { Relaxation of bronchial } \\
\text { smooth muscle }\end{array}$ & $\begin{array}{l}\text { Moderate to severe } \\
\text { asthma }\end{array}$ & $\begin{array}{l}\text { Salmeterol, formoterol, } \\
\text { olodaterol }\end{array}$ \\
\hline LAMAs & $\begin{array}{l}\text { Long-term } \\
\text { control } \\
\text { medications }\end{array}$ & $\begin{array}{l}\text { MRs (functional } \\
\text { selectivity for M3R) }\end{array}$ & $\begin{array}{l}\text { Relaxation of bronchial } \\
\text { smooth muscle }\end{array}$ & Uncontrolled asthma & $\begin{array}{l}\text { Tiotropium, umeclidinium, } \\
\text { glycopyrrolate }\end{array}$ \\
\hline LTRAs & $\begin{array}{l}\text { Long-term } \\
\text { control } \\
\text { medications }\end{array}$ & CysLT receptor 1 & $\begin{array}{l}\text { Antagonize actions of } \\
\text { leukotrienes in the } \\
\text { airway }\end{array}$ & $\begin{array}{l}\text { Secondary option for } \\
\text { mild/moderate } \\
\text { persistent asthma }\end{array}$ & $\begin{array}{l}\text { Montelukast, pranlukast, } \\
\text { zafirlukast }\end{array}$ \\
\hline $\begin{array}{l}\text { Other leukotriene } \\
\text { modifier }\end{array}$ & $\begin{array}{l}\text { Long-term } \\
\text { control } \\
\text { medications }\end{array}$ & 5-LOX & $\begin{array}{l}\text { Inhibit the formation of } \\
\text { leukotrienes }\end{array}$ & $\begin{array}{l}\text { Secondary option for } \\
\text { mild/moderate } \\
\text { persistent asthma }\end{array}$ & Zileuton \\
\hline Methylxanthines & $\begin{array}{l}\text { Long-term } \\
\text { control } \\
\text { medications }\end{array}$ & $\begin{array}{l}\text { Phosphodiesterase } 3, \\
\text { adenosine receptors }\end{array}$ & $\begin{array}{l}\text { Relaxation of bronchial } \\
\text { smooth muscle }\end{array}$ & $\begin{array}{l}\text { Secondary option for } \\
\text { mild/moderate } \\
\text { persistent asthma }\end{array}$ & Theophylline \\
\hline Mast cell stabilizer & $\begin{array}{l}\text { Long-term } \\
\text { control } \\
\text { medications }\end{array}$ & $\begin{array}{l}\text { Calcium-activated } \\
\text { potassium channels }\end{array}$ & $\begin{array}{l}\text { Inhibit the release of } \\
\text { inflammatory } \\
\text { mediators }\end{array}$ & Mild persistent asthma & Cromolyn \\
\hline $\begin{array}{l}\text { Oral and intravenous } \\
\text { corticosteroids }\end{array}$ & $\begin{array}{l}\text { Quick-relief } \\
\text { (rescue) } \\
\text { medications }\end{array}$ & Glucocorticoid receptors & $\begin{array}{l}\text { Suppression of airway } \\
\text { inflammation }\end{array}$ & Severe asthma & $\begin{array}{l}\text { Prednisolone, prednisone, } \\
\text { methylprednisolone }\end{array}$ \\
\hline SABAs & $\begin{array}{l}\text { Quick-relief } \\
\text { (rescue) } \\
\text { medications }\end{array}$ & $\beta_{2} \mathrm{AR}$ & $\begin{array}{l}\text { Relaxation of bronchial } \\
\text { smooth muscle }\end{array}$ & Severe asthma & Albuterol, levalbuterol \\
\hline SAMAs & $\begin{array}{l}\text { Quick-relief } \\
\text { (rescue) } \\
\text { medications }\end{array}$ & $\begin{array}{l}\text { MRs (no selectivity for } \\
\text { M3R) }\end{array}$ & $\begin{array}{l}\text { Relaxation of bronchial } \\
\text { smooth muscle }\end{array}$ & $\begin{array}{l}\text { Acute asthma } \\
\text { exacerbation }\end{array}$ & Ipratropium, oxitropium \\
\hline Antibodies & $\begin{array}{l}\text { Biologic } \\
\text { agents }\end{array}$ & IgE and ILs & $\begin{array}{l}\text { Reducing inflammation } \\
\text { by blocking IgE and } \\
\text { ILs }\end{array}$ & $\begin{array}{l}\text { Allergic and severe } \\
\text { asthma }\end{array}$ & $\begin{array}{l}\text { Omalizumab, } \\
\text { mepolizumab, } \\
\text { dupilumab }\end{array}$ \\
\hline
\end{tabular}

control asthma symptoms through suppression of airway inflammation. Corticosteroids suppress inflammation mainly by acting on the glucocorticoid receptors to switch off multiple activated inflammatory genes that encode for cytokines, chemokines, adhesion molecules, inflammatory enzymes, and receptors, through reversing histone acetylation via the recruitment of histone deacetylase 2 (Barnes, 2006). Corticosteroids can also switch on transcription of genes encoding $\beta_{2^{-}}$ adrenergic receptors $\left(\beta_{2} \mathrm{AR}\right)$, secretory leukocyte inhibitory protein, and mitogen-activated protein kinase (MAPK) phosphatase-1 (Barnes, 2006, 2010a). These effects may contribute to the anti-inflammatory actions of corticosteroids. Inhaled corticosteroids are the most effective and safe medications for long-term asthma control with adjunct medications such as $\beta_{2}$-agonists (Choby and Lee, 2015).

b. Long-acting $\beta_{2}$-agonists. There are a number of LABAs used in the clinics, which include salmeterol, formoterol, vilanterol, and olodaterol. These medicines have much longer half-life profiles in binding to $\beta_{2^{-}}$ adrenergic receptors within the bronchioles than shortacting $\beta_{2}$-agonists, leading to a longer duration of effect (Walker et al., 2011). LABAs are recommended to be only used in the combination therapy with a corticosteroid, whereas LABAs provide symptomatic relief of asthma, and corticosteroids offer control of the underlying inflammatory pathology, thus reducing recurrence of exacerbations and overall mortality (Calverley et al., 2007; Beasley et al., 2010; Weatherall et al., 2010). Common combination therapy medicines include salmeterol/fluticasone, formoterol/budesonide, formoterol/ mometasone, and vilanterol/fluticasone. Formoterol in combined use with budesonide is also used as a reliever medication because of its quick onset of action (O'Byrne et al., 2005).

c. Long-acting muscarinic antagonists. LAMAs include tiotropium, aclidinium, glycopyrronium, and umeclidinium. Muscarinic antagonists elicit their function by binding to muscarinic receptors (MRs) M1, M2, and M3 and blocking neuronal and non-neuronal signals of acetylcholine. Among them, M3 receptors are found on the airway smooth muscle cells, mucosal glands, and vascular endothelium along the airways. Blockage of these receptors leads to a decrease in bronchoconstriction, leading to the primary effects of LAMAs in asthma (Alagha et al., 2014; Cruz, 2018). These LAMAs dissociate more slowly from the M3 receptors than from the $\mathrm{M} 2$ receptors, exhibiting a functional selectivity. Tiotropium has received Food and Drug Administration approval for the treatment of asthma, whereas others have been used in the treatment of COPD and could be developed toward asthma drugs (Lee et al., 2015; Busse et al., 2016; Ferrando et al., 2017; Gosens and Gross, 2018). LAMAs are used in combination with LABAs for increasing the efficacy 
over monotherapy (Rolla and Brussino, 2018). Commonly used medicines include tiotropium/olodaterol, aclidinium/formoterol, glycopyrronium/indacaterol, and umeclidinium/vilanterol.

d. Leukotriene modifiers and leukotriene receptor antagonists. Currently used leukotriene modifiers include montelukast, zafirlukast, pranlukast, and zileuton. Among them, montelukast, zafirlukast, and pranlukast are leukotriene receptor antagonists (LTRAs) that target G protein-coupled receptors (GPCRs), whereas zileuton is an inhibitor of 5-lipoxygenase (5-LOX) that inhibits the formation of leukotrienes [leukotriene $\mathrm{B}_{4}\left(\mathrm{LTB}_{4}\right)$, leukotriene $\mathrm{C}_{4}\left(\mathrm{LTC}_{4}\right)$, leukotriene $\mathrm{D}_{4}\left(\mathrm{LTD}_{4}\right)$, and leukotriene $\mathrm{E}_{4}\left(\mathrm{LTE}_{4}\right)$ ]. These medications work to antagonize the effects of proinflammatory leukotrienes or to decrease leukotriene production. This results in decreased inflammation and decreased hyperresponsiveness of the airways to immune challenges (Scott and Peters-Golden, 2013). Leukotriene modifiers can be used as alternatives to inhaled corticosteroids for mild to moderate asthma, or used in addition to inhaled corticosteroids for moderate asthma (National Asthma Education and Prevention Program, 2007; Scow et al., 2007).

e. Theophylline. The mechanism of action of theophylline in obstructive lung disease management is poorly understood. It may act as a nonselective inhibitor of phosphodiesterases (PDEs) and a nonspecific adenosine receptor antagonist, leading to the relaxation of smooth muscles of airways (Barnes, 2013). It also has been shown to reduce airway inflammation likely by inhibiting PDE4 and activating histone deacetylase 2, which results in the switching off of activated inflammatory genes. It is an alternative medicine to inhaled corticosteroids for mild asthma (Mahemuti et al., 2018). Theophylline can also be used in combination with inhaled corticosteroids to treat moderate to severe asthma (Mahemuti et al., 2018).

f. Cromolyn. Cromolyn is traditionally described as a mast cell stabilizer, yet its mechanism of action is not fully understood. It is known to inhibit calciumactivated potassium channels and thus may inhibit the exaggerated neuronal reflexes triggered by the stimulation of irritant receptors on sensory nerve endings in exercise-induced asthma (EIA). It also inhibits the release of preformed cytokines from several types of inflammatory cells in allergen-induced asthma (Larsson et al., 2001; Yazid et al., 2009). Cromolyn therapy has been largely replaced by LTRAs.

2. Quick-Relief (Rescue) Medications.

a. Oral and intravenous corticosteroids. Oral and intravenous corticosteroids include prednisolone, prednisone, and methylprednisolone. These medications are used on a short-term basis to relieve airway inflammation in severe asthma, as they can cause serious side effects when used long-term (National Asthma Education and Prevention Program, 2007; Albertson et al., 2015; Sharma et al., 2019). b. Short-acting $\beta_{2}$-agonists. SABAs used in North America include albuterol and levalbuterol. Albuterol and levalbuterol are both $\beta_{2}$-adrenergic receptor agonists. Albuterol became commercially available in 1969 and is still used as a highly effective rescue therapy today (Pollock et al., 2017). These medicines function by a similar mechanism of action as LABAs, but with a shorter duration of action. Other SABAs such as terbutaline and procaterol are also used in regions such as Scandinavia and Japan.

c. Short-acting muscarinic antagonists. The commonly used SAMAs are ipratropium and oxitropium. They are derivatives of the nonspecific muscarinic antagonist atropine, which acts by inhibiting the signaling of MRs to relax smooth muscles (Albertson et al., 2015; Pollock et al., 2017). They can also be used in combination with SABAs, such as albuterol or fenoterol, for increasing the efficacy over monotherapy (Bryant and Rogers, 1990; Nishi et al., 1993; Donohue et al., 2016).

3. Biologic Agents. Biologic agents as therapies for asthma were developed rather recently. They target IgE and type 2 cytokines, which can provide additional therapeutic benefits by further reducing type 2 inflammation in asthma. Biologic drugs are usually used as a phenotype-selective treatment method, and our understanding of asthma endotyping helps to identify optimal biologics as targeted therapy (Pelaia et al., 2012; Svenningsen and Nair, 2017).

Omalizumab is a human monoclonal antibody. It works by directly binding and blocking IgE, which is responsible for many allergy symptoms, thus reducing the ability of $\operatorname{IgE}$ to induce a $\mathrm{T}_{\mathrm{H}} 2$ immune response, including eosinophilia and mast cell activation (Chazan, 2001; D'Amato, 2006; Dantzer and Wood, 2018). Omalizumab may be particularly useful for patients who have allergies and severe asthma (National Asthma Education and Prevention Program, 2007; Dantzer and Wood, 2018).

Anti-IL antibodies include mepolizumab, reslizumab, benralizumab, and dupilumab (Colice, 2011; Braido et al., 2015; Farne et al., 2017). These medications are used as add-on therapies for adults with severe asthma and eosinophilia. Mepolizumab, reslizumab, and benralizumab bind to the IL-5 receptor, thus inhibiting its signal to proliferate eosinophils in bone marrow. Dupilumab binds to the IL-4 receptor, modulating signaling of both the IL-4 and IL-13 pathways (Castro et al., 2018).

\section{Inhalation Therapy}

Corticosteroids have been administered for over 50 years and are used to prevent airway inflammation, decrease mucus production, and reduce airway hypersensitivity (Barnes, 2010a). The most commonly prescribed medication for asthma management is an inhaled corticosteroid (ICS) paired with a bronchodilator 
(Barnes, 2010a; Silva and Jacinto, 2016; Gosens and Gross, 2018). Typically the bronchodilator is a SABA or LABA; however, several anticholinergics have been recently approved for asthma management as standalone or add-on therapies. Inhaled therapies are administered by one of three common types of inhalation devices, with the metered dose inhaler (MDI) being the most common. MDIs use a chemical propellant to push the medication out of the inhaler. MDIs often come with what is called a spacer. The spacer helps a person to better coordinate inhalation with release of the drug from the canister and insures that more drug is properly delivered. Dry powdered inhalers do not use a chemical propellant, but require fast and accurate inhalation to ensure that most of the drug enters the lungs. The last type is a nebulizer, which requires the use of a mask that fits over the mouth or nose and requires the use of oxygen or air under pressure to deliver the drug in a fine mist. Nebulizers are the most effective form of drug delivery and especially useful for young children and those who are unable to coordinate inhalation with drug release. However, they are the most time consuming as it can take upward of 15 minutes to deliver each dose of drug.

\section{Preclinical Models of Asthma}

Numerous animal models have been used to decipher various aspects of asthma pathophysiology, identify novel therapeutic targets, and test the efficacy of emerging therapies. Reports using models of Drosophila melanogaster (Roeder et al., 2009), canines (Padrid, 1992; Zosky and Sly, 2007), felines (Norris Reinero et al., 2004), rats (Kucharewicz et al., 2008), equines (Herszberg et al., 2006), sheep (Zosky and Sly, 2007; Scheerlinck et al., 2008), guinea pigs (Canning and Chou, 2008), nonhuman primates (Coffman and Hessel, 2005), and mice (Zosky and Sly, 2007; Nials and Uddin, 2008; Chapman et al., 2014; Aun et al., 2017) can be found in the literature. Although felines and equines spontaneously display asthma-like symptoms, $1 \%$ of cats develop eosinophilic bronchitis (Padrid, 2000; Aun et al., 2017), and horses develop heaves resulting from moldy hay (Barton and Gehlen, 2016); they are not financially viable models for asthma research. Despite the physiologic compatibility of cats and horses, as well as guinea pigs, and nonhuman primates compared with mice, the diminished feasibility based on costs, transgenic resources, and other factors has made mice the gold standard for models of asthma (Zosky and Sly, 2007; Nials and Uddin, 2008; Chapman et al., 2014; Aun et al., 2017).

Although mice are the predominant species used for preclinical models of asthma, there are some distinct physiologic differences and caveats that should be realized. The most obvious is that mice are quadrupeds and the distribution of ventilation is strongly influenced by gravity, which is an important pathophysiological feature of human asthma. Airway branching in mice is also different than humans. Mice only have six to eight distinct branches, whereas humans have 23 that are symmetrical and dichotomous. Branching in mice and other nonprimates is monopodial, in which one daughter branch may be larger than the other (Gomes and Bates, 2002). Compared with humans, mice have large caliber airways for their respective lung size. These differences are likely to affect ventilation and aerosol distribution, thus confounding AHR studies and the implications of airway obstruction and structural changes, as well as the effects of nonaerosolized treatments (Chapman et al., 2014).

As mice do not spontaneously develop allergic airway disease, models have been developed that include sensitization with an allergen, often accompanied by an adjuvant, followed by multiple challenges with allergen alone. A common timeline involves sensitization in the first 2 weeks, followed by challenge at multiple time points during the following weeks for acute studies and challenge over the course of 13 months for chronic models. Much like the choice of species, other factors including time and expense have resulted in the majority of studies being designed using acute models, which does not best reflect what occurs clinically (Nials and Uddin, 2008; Aun et al., 2017). Chronic models have been developed to better replicate structural changes that include subepithelial and airway wall fibrosis, goblet cell hyperplasia, smooth muscle thickening, and increased vascular permeability (Nials and Uddin, 2008; Aun et al., 2017). Chronic inflammation and structural changes have functional consequences that contribute to asthma symptoms and reflect the clinical phenotype, as most result in airway remodeling and persistent AHR. However, there are also concerns regarding chronic models, as it has been observed that immune tolerance may develop (Aun et al., 2017; Sethi and Naura, 2018). Additionally, mouse inflammation is not restricted to the conducting airways as it is in humans, and both lung parenchymal and vascular inflammation and remodeling are observed in mice as well (Nials and Uddin, 2008). There is also a disparity in the recruitment of mast cells to airway walls and epithelium, which likely speaks to the paucity of mast cells in mice (Lei et al., 2013), making the role of the prostaglandin $(\mathrm{PG}) \mathrm{D}_{2}$ receptor, $\mathrm{DP} 2$, or therapies directed toward them challenging to study in mice.

Mouse strain, allergen, route of administration for sensitization and challenge, and timing are all aspects of a model that will influence the inflammatory response. BALB/c mice are often used as they present a $\mathrm{T}_{\mathrm{H}} 2$-biased response; however, $\mathrm{C} 57 \mathrm{BL} / 6$ and $\mathrm{A} / \mathrm{J}$ mouse models have also been developed. For instance, C57BL/6 are a common background for transgenic mice, and this strain is also used in high-fat-diet asthma models as male C57BL/6 more readily develop a type 2 
diabetic phenotype (Kim et al., 2014). A/J mice have been used in several studies modeling steroid resistance (Serra et al., 2018).

Ovalbumin (OVA) challenge has been the mainstay allergen of the allergic airway disease model. Mice are sensitized with OVA and $\mathrm{Al}(\mathrm{OH})_{3}$ adjuvant, most often via intraperitoneal injection within the first 2 weeks, followed by a period of challenge via oral pharyngeal (intratracheal), intranasal, or aerosolized delivery (Nials and Uddin, 2008; Chapman et al., 2014; Aun et al., 2017). The use of OVA to induce allergic airway disease has been heavily criticized as intraperitoneal administration is not the natural route of allergen exposure, and OVA is not a clinically relevant allergen (Chapman et al., 2014). More recently, models have developed in which house dust mite (HDM), cockroach extract (Blatella germanica, $\mathrm{Bl}$ g2), ragweed, Aspergillus fumigatus (fungi causing allergic rhinitis), and the parasite Ascaris lumbricoids have been used (Zosky and Sly, 2007; Nials and Uddin, 2008; Chapman et al., 2014; Hawlader et al., 2014; Aun et al., 2017). Studies indicate that $50 \%$ to $85 \%$ of asthmatics are allergic to HDM (Nelson et al., 1996), and, although HDM is typically administered without an adjuvant during sensitization, it must be noted that endotoxin levels can vary between lots and sometimes be quite high. This must be taken into consideration as the lipopolysaccharide present will promote an acute inflammatory response. Along with lipopolysaccharide, HDM protein, fecal matter, and chitin found in the HDM extract induces TSLP, granulocyte-macrophage colony-stimulating factor, IL-25, and IL-33 via TLR4 activation on airway epithelial cells (Hammad et al., 2009).

Studies using gene knockout mice combined with OVA challenge have helped to establish the roles of muscarinic acetylcholine receptors in asthma (Kistemaker et al., 2014). A number of studies used OVA-challenged mice and guinea pigs to test the effects of tiotropium, a classic LAMA drug, in the airway inflammation, which suggested a combined therapy of tiotropium and a steroid in the treatment of asthma (Buels et al., 2012; Kang et al., 2012; Kistemaker et al., 2016). OVAchallenged mice models have also been used to examine the role of CysLT receptors and leukotriene receptor antagonists in airway inflammation and modeling (Henderson et al., 2002; Ikeda et al., 2014). Interestingly, a few studies using gene knockout mice with OVA or leukotriene $\mathrm{E}_{4}$ challenge have predicted additional leukotriene receptors that may be critical new drug targets for asthma, which are still under investigation (Paruchuri et al., 2009; Kanaoka et al., 2013; Trinh et al., 2019).

Utilization of preclinical models of asthma has been invaluable to the characterization of the $\mathrm{T}_{\mathrm{H}} 2$-high atopic phenotype, the discovery of novel targets, and determination of drug efficacy, despite the many caveats described. An in-depth understanding of the mechanisms underlying each asthma phenotype remains the ultimate path forward in personalized medicine, and mouse and other animal models of asthma can be tailored to specific aspects that contribute to these phenotypes. For instance, animal models provide an opportunity to study parameters aside from allergens that contribute to the asthma pathophysiology, including obesity, age, steroid resistance, aspirin sensitivity, and asthma severity. Despite their utility, animal model research should not directly lead to clinical conclusions, but inform us of important targets for therapy and novel pathways of interest. Models should be paired with studies involving clinical samples, such as those conducted using bronchoscopy-derived primary airway epithelial cells at the air-liquid interface, precision-cut lung slices, or the reprogramming of patient-derived pluripotent stem cells and fibroblasts to epithelial and smooth muscle cells (Mullane and Williams, 2014). These types of translational studies will provide a more comprehensive understanding of the complex interactions of inflammation, bronchodilation, and AHR that present as asthma.

\section{E. G Protein-Coupled Receptors as Important Drug Targets in Asthma}

GPCRs constitute the largest family of drug targets in humans. It was estimated that over $33 \%$ of currently used small-molecule drugs target GPCRs (Santos et al., 2017). This is due to many druggable properties of GPCRs. First, GPCRs are key players that sense extracellular signaling molecules on cell surfaces. They are involved in nearly every aspect of in vivo physiologic processes. Almost every physiologic pathway can be dampened or enhanced by pharmacologically manipulating certain GPCRs. Second, most native and endogenous GPCR ligands are small-molecule chemicals and peptides, and many GPCR-targeting drugs were developed based on natural molecules. Thus, there are many natural chemical templates for designing novel small-molecule drugs. Lastly, the ligand-binding sites are located at the extracellular regions of GPCRs, which can be easily accessed by drugs.

Many currently used asthma medications target GPCRs. Studies in the past several decades have proven the important roles of a number of GPCRs, including $\beta_{2}$-adrenergic receptor, muscarinic acetylcholine receptors, leukotriene and PG receptors, chemokine receptors, and adenosine receptors in asthma. A large number of clinical trials have provided critical insights into the significance of GPCR-targeting therapies in the treatment of asthma. Also, the progress in GPCR structural biology in the past decade has allowed us to understand the actions of many GPCR-targeting drugs on a molecular level (Hauser et al., 2017). In this review, we will focus on the GPCRs as existing and potential drug targets for asthma. We hope to 
summarize our current understanding of their physiology and pharmacology and discuss structural insights into drug action.

\section{II. $\boldsymbol{\beta}_{\mathbf{2}}$-Adrenergic Receptor and $\boldsymbol{\beta}_{\mathbf{2}}$-Agonists}

\section{A. Pathophysiological Roles and Signaling of the $\beta_{2}$ - Adrenergic Receptor in Asthma}

The $\beta_{2} \mathrm{AR}$ is a member of the adrenergic receptor (adrenoceptor) family, which is comprised of two major $\alpha$ types and three $\beta$ types of receptors. These receptors are targets of catecholamine neurotransmitters, including epinephrine and norepinephrine (adrenaline and noradrenaline). Catecholamine-signaling molecules act on these receptors to stimulate the sympathetic nervous system to mediate classic fight-or-flight responses. Members of this family are all important drug targets. $\beta_{2} \mathrm{AR}$ is highly expressed in airway smooth muscle (ASM) cells and bronchial epithelial cells. $\beta_{2}$-agonists have been used as bronchodilators in the treatment of various respiratory diseases, including asthma, for decades.

Historically, the $\beta_{2} \mathrm{AR}$ has been the model receptor for GPCR-related research. Hamster $\beta_{2} \mathrm{AR}$ was the first GPCR to be cloned in the late 20th century (Dixon et al., 1986; Lefkowitz, 2004). Results from studies of the $\beta_{2} \mathrm{AR}$ have greatly advanced our understanding of GPCR pharmacology and signaling. In the classic scenario of receptor signaling, the $\beta_{2} \mathrm{AR}$ can be activated by endogenous agonists, epinephrine, and norepinephrine, or synthetic agonists, such as $\beta_{2}$-agonist drugs, to couple to and further activate the stimulatory $\mathrm{G}_{\mathrm{s}}$ protein, resulting in the release of the $\alpha$ subunit of $\mathrm{G}_{\mathrm{s}}$ $\left(\mathrm{G} \alpha_{\mathrm{s}}\right)$. The $\mathrm{G} \alpha_{\mathrm{s}}$ then binds to and activates adenylyl cyclase, which in turn catalyzes the conversion of ATP to cAMP.

cAMP is a second-messenger molecule that can induce cAMP-dependent signaling pathways. One of the well-characterized cAMP effectors is protein kinase A (PKA). PKA was the first cAMP effector to be discovered and is a major effector in asthma airway pathophysiology (Torphy, 1994; Hanoune and Defer, 2001; Beavo and Brunton, 2002), although the roles of PKA in mediating $\beta_{2}$-agonist-mediated relaxation of airways are still controversial. Spicuzza et al. (2001) showed that the suppression of acetylcholine-induced contractions by $\beta_{2}$-agonists is independent of PKA. Another study by Morgan et al. (2014) suggested that $\beta_{2}$-agonistmediated relaxation of airways is PKA dependent. It has been established that PKA phosphorylates many substrates in the ASM cells, such as myosin light chain kinase, inositol triphosphate receptors, calcium-gated potassium channels, and receptors that mediate $G_{q}$ signaling pathways. The consequences of these PKAmediated events include the downregulation of intracellular calcium levels and the reduction of sensitivity of contractile proteins to calcium, which overall counteract mechanisms that lead to ASM contraction (Penn, 2008;
Walker et al., 2011). However, it is likely that different mechanisms are involved in promoting the relaxation of airways and protecting relaxed airways against contractile stimuli. In addition, PKA has been demonstrated to mediate the antimitogenic effects of $\beta_{2^{-}}$ agonists (Yan et al., 2011).

Another cAMP effector involved in airway physiology are Epac proteins, the exchange factors directly activated by cAMP (Roscioni et al., 2008). Epac 1 and 2 have been characterized to be the cAMP-dependent nucleotide exchange factors for Rap proteins and several others of the RAS-like small GTPase family. As novel cAMP sensors, Epac proteins regulate diverse cellular processes. The involvement of Epac proteins in asthma remains elusive. Some studies suggest that cAMPmediated signaling through Epac contributes to the relaxation of contracted smooth muscle (Zieba et al., 2011). Further investigation into the roles of Epac proteins in the physiologic function of $\beta_{2}$-agonists will help to determine whether they are valid therapeutic targets for asthma.

Agonist-activated $\beta_{2} \mathrm{AR}$ can be phosphorylated by many kinases, including PKA and GPCR kinases, which leads to the recruitment of $\beta$-arrestins and the initiation of receptor internalization. $\beta$-arrestins were originally considered to be the terminators of $\mathrm{G}$ proteindependent signaling because they can competitively block the coupling of G proteins to GPCRs. In the late 1990 s and early 2000s, Luttrell et al. (1999), Shenoy and Lefkowitz (2005), and Shenoy et al. (2006) provided evidence suggesting that $\beta_{2} \mathrm{AR}$ could also signal through $\beta$-arrestins, and the $\beta$-arrestin-mediated pathways were $G$ protein-independent. This has led to the establishment of concept of biased signaling or functional selectivity that applies to many other GPCRs. Biased signaling implies that different agonists may act on the same receptor, but recruit and activate distinct signaling partners, including $\mathrm{G}$ proteins and $\beta$-arrestins, to induce disparate signaling events (DeWire et al., 2007; Smith and Rajagopal, 2016).

Although the signaling of $\beta_{2} \mathrm{AR}$ can relax the airways to relieve asthma symptoms, it can also promote inflammation and other detrimental effects in asthma. Knight et al. (2015) showed that LABAs such as salmeterol could promote the activation of STAT6, an important transcription factor involved in the activation of $\mathrm{T}_{\mathrm{H}} 2$ cells, leading to the enhancement of asthma-like allergic airway disease in mice. Consistently, using $\beta_{2} \mathrm{AR}^{-/-}$mice, another study demonstrated that the signaling of $\beta_{2} \mathrm{AR}$ in airway epithelial cells could lead to AHR and promote eosinophilic inflammation and mucous cell metaplasia (Nguyen et al., 2017). The same study also suggested the involvement of $\beta$-arrestins, in particular $\beta$-arrestin- 2 , in the $\beta_{2}$ AR-induced inflammation.

$\beta$-arrestins mediate largely unwanted effects of $\beta_{2^{-}}$ agonists in the treatment of asthma. It is well known 
that long-term treatment with $\beta_{2}$-agonists can lead to desensitization or tolerance, which may partly stem from the $\beta$-arrestin-mediated internalization and degradation of $\beta_{2} \mathrm{AR}$ (Violin et al., 2008; Penn et al., 2014). In addition, $\beta$-arrestins, especially $\beta$-arrestin- 2 , have been shown to constrain $\beta_{2}$-agonistinduced relaxation of ASM, which is G proteindependent (Deshpande et al., 2008). Previous studies from Bond, Walker, and colleagues (Walker et al., 2003; Hollingsworth et al., 2010; Penn et al., 2014), particularly those based on $\beta$-arrestin- $2^{-1-}$ mice, suggested that $\beta$-arrestin-dependent signaling promotes inflammation in the airways. In fact, $\beta$-arrestin-dependent signaling pathways induced by GPCRs expressed in the airway cells have been shown to contribute significantly to the development of asthma, which may involve not only $\beta_{2} \mathrm{AR}$, but also other GPCRs (Walker et al., 2003; Hollingsworth et al., 2010; Walker and DeFea, 2014).

Besides airway cells, B cells (Sanders, 2012) and several innate immune cells, including eosinophils (Yukawa et al., 1990) and mast cells (Chong et al., 2002), also express $\beta_{2} \mathrm{AR}$. The expression of $\beta_{2} \mathrm{AR}$ in different T cell subtypes is contentious (Sanders et al., 1997; Loza et al., 2006). The signaling of $\beta_{2} \mathrm{AR}$ in the immune cells in the context of asthma and airway inflammation is less studied and defined. The role of $\beta_{2} \mathrm{AR}$ signaling in innate immune cells seems to be antiinflammatory. A SABA, albuterol, has been shown to reduce the secretion of $\mathrm{LTC}_{4}$ and eosinophil peroxidase by eosinophils (Munoz et al., 1994). $\beta_{2} \mathrm{AR}$ signaling and $\beta_{2}$-agonists could also inhibit the release of histamine and other inflammatory mediators from human mast cells in lung and peripheral blood (Chong et al., 2002; Wang and Lau, 2006; Scola et al., 2009). In contrast, $\beta_{2} \mathrm{AR}$ signaling in $\mathrm{B}$ cells has been suggested to promote IgE production (Coqueret et al., 1995; Kasprowicz et al., 2000), which is dependent on the activation of p38 MAPK, but not PKA (Pongratz et al., 2006; McAlees and Sanders, 2009), thus contributing to airway inflammation. In addition, Loza et al. (2007) showed that a nonselective $\beta$-agonist can enhance the survival and accumulation of $\mathrm{T}_{\mathrm{H}} 2$ cells through PKA-independent mechanisms.

Because of the proinflammatory side effects of $\beta_{2} \mathrm{AR}$ signaling, even though antagonists or inverse agonists of $\beta_{2} \mathrm{AR}$ such as commonly used $\beta$-blockers were contraindicated in the past in asthma because of their potential effects of worsening bronchospasm (Olenchock et al., 2009), they may provide therapeutic benefits in asthma to reduce inflammation and AHR and/or resensitize $\beta_{2} \mathrm{AR}$ (Dickey et al., 2010). Several studies from Bond and colleagues (Callaerts-Vegh et al., 2004; Joshi et al., 2017) showed the beneficial effects of certain $\beta$-blockers in murine models of asthma. The biased signaling properties of $\beta$-blockers may play important roles in this context because several $\beta$-blockers such as carvedilol have been shown to induce $\beta$-arrestin-dependent signaling and MAPK activation, which promote inflammation (Galandrin and Bouvier, 2006; Wisler et al., 2007; Thanawala et al., 2014). However, the different results from clinical studies using two $\beta$-blockers, nadolol (Hanania et al., 2008, 2010) and propranolol (Short et al., 2013), have spurred a large debate over whether functionally selective $\beta$-blockers represent a potential therapeutic method for asthma (Kazani and Israel, 2013, 2014; Bond, 2014; Lipworth et al., 2014, 2016; Penn, 2014). The safety of using $\beta_{2}$ AR-targeting $\beta$-blockers in asthma patients is also controversial and needs to be further investigated (Arboe and Ulrik, 2013; Morales et al., 2017). More clinical investigation and data are needed to clarify if and under what conditions $\beta$-blockers do provide therapeutic benefits over potential risks in the treatment of asthma.

\section{B. Currently Used $\beta_{2}$-Agonist Drugs and Their Pharmacological Properties}

More than 100 years ago, researchers already found that the endogenous ligand of $\beta_{2} \mathrm{AR}$, epinephrine, could be injected into patients to acutely treat an asthma attack. In the mid-20th century, studies suggested that inhalation of epinephrine also worked well in releasing bronchoconstriction (Middleton and Finke, 1968). This has led to the wide use of inhalers for delivering $\beta_{2^{-}}$ agonists as bronchodilators for treating asthma and COPD. In the past eight decades, a large number of $\beta_{2^{-}}$ agonists have been developed and approved for this. The first synthetic $\beta_{2}$-agonist, isoproterenol, was introduced to the clinic in the 1940s. Further medicinal chemical studies led to the development of a number of $\beta_{2}$-agonists in the $1960 \mathrm{~s}$ and $1970 \mathrm{~s}$ as the first generation of $\beta_{2}$-agonist drugs, some of which are still used today as the mainstream $\beta_{2}$-agonist drugs such as salbutamol, formoterol, and salmeterol. New $\beta_{2^{-}}$ agonists are still being developed for the asthma market, and a detailed discussion of the clinical use of $\beta_{2}$-agonists can be found in a recent review article (Billington et al., 2017).

Based on their duration of action, traditional $\beta_{2^{-}}$ agonists were classified into two groups: short-acting $\beta_{2}$-agonists (SABAs), such as isoproterenol, salbutamol, and terbutaline, and LABAs, such as salmeterol and formoterol. SABAs provide almost instant relief of asthma symptoms and are used as rescue medicines for treating acute asthma attacks. However, their bronchodilatory effects usually last for only a few hours, thus limiting their use on a regular basis. LABAs exhibit a long duration of action for more than 12 hours and are commonly used with ICS for the long-term management of asthma symptoms. In the past decade, new agents have been developed as ultra-LABAs, extending the duration of action to over 24 hours for the COPD market. These potent Food and Drug Administration (FDA)-approved bronchodilators include 
indacaterol, olodaterol, and vilanterol. The use of ultraLABAs has led to improved clinical outcomes as the once-daily dosing regimen has likely improved medication adherence (Monaco and Hanania, 2017). Whether ultra-LABAs can provide similar clinical benefits to asthma patients is still under investigation.

A major aspect of the research efforts that have gone into the development of new $\beta_{2}$-agonists is focused on improving duration of action. However, other pharmacological properties of LABAs and ultra-LABAs also affect their clinical outcomes. One of them is the receptor subtype selectivity, which may be associated with the cardiac side effects of $\beta_{2}$-agonists (Salpeter et al., 2004). Three subtypes of $\beta$-adrenergic receptors have been characterized to date, $\beta_{1}$-adrenergic receptor $\left(\beta_{1} \mathrm{AR}\right), \beta_{2} \mathrm{AR}$, and $\beta_{3}$-adrenergic receptor ( $\left.\beta_{3} \mathrm{AR}\right) . \beta_{2} \mathrm{AR}$ is the dominant $\beta$-adrenergic receptor expressed in the airways, and $\beta_{1} \mathrm{AR}$ and $\beta_{2} \mathrm{AR}$ are both highly expressed in cardiac tissues with an estimated ratio of $3: 1$ (Bristow, 2000). $\beta_{3} \mathrm{AR}$ is much less studied, but it is believed to be mainly located in adipose tissues to regulate lipolysis and energy expenditure. Overstimulation of $\beta_{1} \mathrm{AR}$ and $\beta_{2} \mathrm{AR}$ in the heart can induce adverse cardiovascular events. Also, due to the sequence similarity between $\beta_{1} \mathrm{AR}$ and $\beta_{2} \mathrm{AR}$ and the conservation of the ligand-binding sites, most of the ligands acting on these two receptors do not exhibit high receptor subtype selectivity, making cardiovascular side effects a major concern (Baker, 2005, 2010). Indeed, a meta-analysis suggested that many $\beta_{2}$-agonists were associated with an increased risk of adverse cardiovascular events, leading to myocardial infarction, arrhythmias, ischemia, and heart failure through the stimulation of $\beta$-adrenergic receptors (Cazzola et al., 2005). In contrast, because $\beta_{1} \mathrm{AR}$ is expressed with a much higher level in the heart than it of $\beta_{2} \mathrm{AR}$, highly selective $\beta_{2} \mathrm{AR}$ agonists may be associated with a lower cardiac toxicity compared with nonselective $\beta_{2}$-agonists. This was confirmed by clinical studies on a long and widely used LABA, salmeterol, which exhibits over 1000-fold selectivity over the $\beta_{1} \mathrm{AR}$ (Ferguson et al., 2003). In these studies, regular use of salmeterol minimally increased the risk of adverse cardiovascular events compared with placebo (Ferguson et al., 2003). Another small-scale clinical study examined the cardiac effects of salmeterol and another LABA, formoterol (Cazzola et al., 1998). Compared with salmeterol, formoterol has a much lower selectivity for the $\beta_{2} \mathrm{AR}$ (Baker, 2010), and this study showed that formoterol used at a dosage of $24 \mu \mathrm{g} /$ day was associated with a higher heart rate than $50 \mu \mathrm{g} /$ day salmeterol.

The lower receptor subtype selectivity is not the only reason for the stronger cardiac effects associated with formoterol compared with salmeterol. Another pharmacological property of $\beta_{2}$-agonists that may affect their therapeutic effects is efficacy. Data from biophysical studies on purified $\beta_{2} \mathrm{AR}$ showed that salmeterol is less efficacious in inducing a fully active conformation of the receptor for $G_{\mathrm{s}}$ coupling compared with full agonists, epinephrine and isoproterenol, indicating a partial agonism of salmeterol in this minimized, receptor-only system (Gregorio et al., 2017; Masureel et al., 2018). Consistently, it has been shown that in the heterologous expression systems and native cells formoterol is a full agonist of $\beta_{2} \mathrm{AR}$ with a similar efficacy as isoproterenol, and salmeterol is a partial agonist with lower efficacy at saturating concentrations in the context of cellular $G_{s}$ signaling and cAMP accumulation (McCrea and Hill, 1993; Bouyssou et al., 2010). Another study measuring relaxation of isolated human bronchi also showed that salmeterol is a weaker $\beta_{2}$-agonist than formoterol (Naline et al., 1994). Clinical studies confirmed the partial agonism of salmeterol in reducing bronchoconstriction in asthma patients compared with formoterol (Palmqvist et al., 1997, 1999). In another clinical study comparing the cardiac effects of formoterol and salmeterol, the authors suggested that the lower efficacy of salmeterol contributes to its decreased cardiac effects (Cazzola et al., 1998). It was proposed that partial agonists in general might provide more benefits for treating chronic diseases by minimizing the overstimulation and desensitization of cell surface receptors compared with full agonists (Zhu, 1996).

It has to be pointed out that several studies also demonstrated a maximal functional efficacy of salmeterol in systems in which there is a high receptor density or an efficient coupling to adenylyl cyclase (Cooper et al., 2011; Yan et al., 2018). Such an inconsistency suggests the importance of experimental contexts in measuring ligand efficacy. This is not just for $\beta_{2} \mathrm{AR}$, but also for many other GPCRs. The traditional concept of ligand efficacy may need to be revisited given the high complexity and heterogeneity of GPCR signaling systems revealed by recent research progress. Nevertheless, salmeterol is still considered as a potent bronchodilator with satisfactory clinical efficacy, as evidenced by its wide use (Cazzola et al., 2013). Similarly, a SABA, salbutamol, is also a partial agonist of $\beta_{2} \mathrm{AR}$ that is widely used for treating asthma attacks.

The concept of biased signaling is relatively new, but is another important aspect that may contribute to $\beta_{2}$-agonist mechanism of action, although controversies still exist. The biased property of $\beta_{2}$-agonists has been extensively studied in heterologous systems. van der Westhuizen et al. (2014) showed that several $\beta_{2^{-}}$ agonists including salbutamol and salmeterol exhibit significant signaling bias toward extracellular signalregulated kinase 1/2 pathway over cAMP accumulation, calcium mobilization, and receptor endocytosis relative to isoproterenol in HEK293 cells. Studies from Clark and others (Carter and Hill, 2005; Moore et al., 2007; Gimenez et al., 2015; Masureel et al., 2018) demonstrated that salmeterol behaved as a biased ligand against $\beta$-arrestin recruitment and receptor 
desensitization relative to formoterol or epinephrine in assays using transfected cells, although another study suggested similar efficacies of salmeterol for $\mathrm{G}_{\mathrm{s}}$-dependent signaling and $\beta$-arrestin-associated signaling (Drake et al., 2008). Littmann et al. (2015) tested 40 $\beta_{2}$-agonists in transfected HEK293 cells and reported a bias toward $\mathrm{G}_{\mathrm{s}}$ signaling over $\beta$-arrestin recruitment for many agonists, including salmeterol and fenoterol, relative to isoproterenol and formoterol. However, whether commonly used $\beta_{2}$-agonists such as salmeterol exhibit functional bias in native systems still needs further investigation.

Intuitively, poor desensitization of $\beta_{2} \mathrm{AR}$ may contribute to the prolonged therapeutic effects of $\beta_{2^{-}}$ agonists. Early studies suggested that partial agonists with low efficacy such as salmeterol cause less receptor desensitization compared with full agonists such as formoterol (January et al., 1997, 1998). However, later studies provided evidence suggesting a much more complex scenario, in which the pattern and mechanism of receptor desensitization are agonist-specific (Düringer et al., 2009; Cooper et al., 2011). Clinical studies on the receptor desensitization induced by $\beta_{2^{-}}$ agonists generated contradictory results, which were reviewed in a commentary by Charlton (2009).

It needs to be emphasized that the use of $\beta_{2}$-agonists alone can lead to serious detrimental effects (Cockcroft and Sears, 2013). Historically, long-term overuse of isoproterenol alone as a SABA was linked to increased asthma mortality in some countries in the mid 20th century (Inman and Adelstein, 1969). A meta-analysis also suggested an increased risk of asthma mortality associated with salmeterol monotherapy (Weatherall et al., 2010). The same analysis also suggested that concomitant use of ICS could mitigate such risk. The underlying mechanism is still not entirely understood. Considering the fact that ICS reduces lung inflammation, it is possible that the inflammatory effects of $\beta_{2} \mathrm{AR}$ signaling are the major determinant for the increased risk of asthma mortality associated with chronic $\beta_{2^{-}}$ agonist therapy. Indeed, $\beta_{2}$-agonists have been shown to induce inflammatory mediators in airway epithelial cells, which may be through $\mathrm{G}_{\mathrm{s}}$ signaling (Edwards et al., 2007; Ritchie et al., 2018). A recent study analyzed gene expression changes caused by $\beta_{2}$-agonists and showed that an ultra-LABA, indacaterol, could upregulate many genes encoding proinflammatory proteins, most likely through the canonical cAMP/ PKA signaling pathway (Yan et al., 2018). As discussed before, $\beta$-arrestin signaling pathways downstream $\beta_{2} \mathrm{AR}$ activation also largely promote inflammation. Interestingly, in contrast to the proinflammatory roles, a number of studies from Newton and colleagues (Kaur et al., 2008b,a; Manetsch et al., 2012) showed that $\beta_{2^{-}}$ agonists could enhance the anti-inflammatory action of glucocorticoids through the cAMP/PKA pathway. As required by the FDA, drugs that contain both a LABA and ICS were evaluated in large clinical trials for the risk of serious asthma outcomes, and the results supported a high safety profile with minimal side effects for such combined therapy (Billington et al., 2017). Therefore, nearly all treatment guidelines recommend that chronic use of LABAs should always be combined with an ICS.

\section{Structural Insights into Drug Action}

The $\beta_{2} \mathrm{AR}$ has served as a model receptor for GPCRrelated studies for more than three decades. There is extensive experimental data providing detailed molecular insights into the activation and signaling of the receptor and the action of various $\beta_{2}$-agonists. Intensive research efforts have been devoted to solving highresolution structures of $\beta_{2} \mathrm{AR}$ through X-ray crystallography approaches, which has led to the deposition of 22 structures in the Protein Data Bank (PDB) (Table 2). These structures revealed inactive and active conformational states of $\beta_{2} \mathrm{AR}$ and the binding sites for a variety of antagonists and agonists.

1. Chemical Structures of $\beta_{2}$-Agonists. Almost all $\beta_{2}$-agonist drugs share a similar chemical scaffold as the endogenous ligand epinephrine, which is characterized by an aromatic group as the head (catechol group in epinephrine), an ethanolamine group as the middle moiety, and a tail group with diverse chemical structures (Fig. 1).

\section{Structural Insights into the Pharmacological} Properties of $\beta_{2}$-Agonists.

a. Ligand efficacy. Results from structural and biophysical studies of $\beta_{2} \mathrm{AR}$ have provided a detailed molecular mechanism for how agonists induce receptor activation (Rasmussen et al., 2011a,b; Ring et al., 2013; Manglik and Kruse, 2017; Hilger et al., 2018; Masureel et al., 2018). As for almost all other rhodopsin-like class A GPCRs (Fredriksson et al., 2003), the ligand-binding pockets in the $\beta_{2} \mathrm{AR}$ are located within the helical bundle formed by seven-transmembrane helices (7TMs). Compared with the inactive $\beta_{2} \mathrm{AR}$ with antagonists and inverse agonists, the binding of agonists at the extracellular region can cause a contraction of the ligand-binding pocket with a slight inward movement of transmembrane helix 5 (TM5), which is linked to large conformational changes at the cytoplasmic surface through a rearrangement of a triad of residues in the middle of the 7TM helical bundle (Rasmussen et al., 2011a). The conformational changes at the cytoplasmic surface include a rotation and large outward movement of TM6, creating an open cavity to accommodate the C-terminal helix of the $\mathrm{G} \alpha_{\mathrm{s}}$. The outward movement of TM6 at the cytoplasmic surface is a hallmark of GPCR activation.

The aromatic head groups of $\beta_{2}$-agonists together with the ethanolamine group are involved in polar interaction networks with nearby polar residues in the ligand-binding pockets that include serine residues 
TABLE 2

Structures of human $\beta_{2} \mathrm{AR}$ bound to various ligands

\begin{tabular}{|c|c|c|c|c|c|c|}
\hline $\mathrm{PDB} \mathrm{ID}^{\alpha}$ & Ligand & Efficacy & Fusion Partner ${ }^{b}$ & Binding Partner & Resolution $(\AA)$ & References \\
\hline 2RH1 & Carazolol & Inverse agonist & $\begin{array}{l}\text { T4 lysozyme }(\mathrm{T} 4 \mathrm{~L}) \\
\text { in ICL3 }\end{array}$ & None & 2.40 & $\begin{array}{l}\text { Cherezov et al., 2007; } \\
\text { Rosenbaum et al., } 2007\end{array}$ \\
\hline 2R4R & Carazolol & Inverse agonist & None & Fab5 & 3.40 & Rasmussen et al., 2007 \\
\hline $2 \mathrm{R} 4 \mathrm{~S}$ & Carazolol & Inverse agonist & None & Fab5 & 3.40 & Rasmussen et al., 2007 \\
\hline 3D4S & Timolol & Inverse agonist & T4L in ICL3 & None & 2.80 & Hanson et al., 2008 \\
\hline 3KJ6 & Carazolol & Inverse agonist & None & Fab5 & 3.40 & Bokoch et al., 2010 \\
\hline $3 N Y 8$ & ICI 118551 & Inverse agonist & T4L in ICL3 & None & 2.84 & Wacker et al., 2010 \\
\hline $3 N Y 9$ & Compound $2 \mathrm{a}^{c}$ & Inverse agonist & T4L in ICL3 & None & 2.84 & Wacker et al., 2010 \\
\hline $3 \mathrm{NYA}$ & Alprenolol & Antagonist & $\mathrm{T} 4 \mathrm{~L}$ in ICL3 & None & 3.16 & Wacker et al., 2010 \\
\hline 4GBR & Carazolol & Inverse agonist & N-terminal T4L & None & 3.99 & Zou et al., 2012 \\
\hline 5D5A & Carazolol & Inverse agonist & T4L in ICL3 & None & 2.48 & Huang et al., 2016a \\
\hline 5D5B & Carazolol & Inverse agonist & T4L in ICL3 & None & 3.80 & Huang et al., 2016a \\
\hline $5 \mathrm{JQH}$ & Carazolol & Inverse agonist & N-terminal T4L & Nanobody Nb60 & 3.20 & Staus et al., 2016 \\
\hline 5D5B & Carazolol & Inverse agonist & T4L in ICL3 & None & 3.20 & Ma et al., 2017 \\
\hline $5 \mathrm{X} 7 \mathrm{D}$ & $\begin{array}{l}\text { Carazolol and intracellular } \\
\text { antagonist Cmpd-15PA }\end{array}$ & $\begin{array}{l}\text { Inverse agonist and } \\
\text { allosteric } \\
\text { antagonist }\end{array}$ & $\mathrm{T} 4 \mathrm{~L}$ in ICL3 & None & 2.70 & Liu et al., 2017 \\
\hline 3PDS & FAUC50 & Covalent agonist & T4L in ICL3 & None & 3.50 & Rosenbaum et al., 2011 \\
\hline 3P0G & BI-167107 & Full agonist & None & Nanobody Nb80 & 3.50 & Rasmussen et al., 2011a \\
\hline 3SN6 & BI-167107 & Full agonist & N-terminal T4L & $\mathrm{G}_{\mathrm{s}}$ heterotrimer & 3.20 & Rasmussen et al., 2011b \\
\hline $4 \mathrm{LDE}$ & BI-167107 & Full agonist & N-terminal T4L & Nanobody Nb6B9 & 2.79 & Ring et al., 2013 \\
\hline 4LDL & Hydroxybenzyl isoproterenol & Full agonist & N-terminal T4L & Nanobody Nb6B9 & 3.10 & Ring et al., 2013 \\
\hline $4 \mathrm{LDO}$ & Adrenaline (epinephrine) & Full agonist & N-terminal T4L & Nanobody Nb6B9 & 3.20 & Ring et al., 2013 \\
\hline $4 \mathrm{QKX}$ & Compound $2 \mathrm{~b}^{c}$ & Covalent agonist & N-terminal T4L & Nanobody Nb6B9 & 3.30 & Weichert et al., 2014 \\
\hline $6 \mathrm{MXT}$ & Salmeterol & Partial agonist & N-terminal T4L & Nanobody Nb71 & 2.96 & Masureel et al., 2018 \\
\hline
\end{tabular}

${ }^{a} \mathrm{PDB}$, https://www.rcsb.org/.

${ }^{b}$ Inserting a small protein into GPCRs either at the ICL3 or at the N-terminal ends has been widely used to facilitate crystallization.

${ }^{c}$ Compound $2 \mathrm{a}$ and compound $2 \mathrm{~b}$ are unrelated. They were both named "compound 2 " in the literature. Here we use $2 \mathrm{a}$ and $2 \mathrm{~b}$ to avoid confusion

ICL3, intracellular loop 3.

Ser203 ${ }^{5.42}$, Ser204 $4^{5.43}$, and Ser207 ${ }^{5.46}$ in TM5; Asn293 $3^{6.55}$ in TM6; Asp $113^{3.32}$ in TM3; and Asn312 7.39 in TM7 (Ballesteros-Weinstein numbering system) (Juan and Ballesteros, 1995) (Fig. 2A) (Supplemental Material 1PDB IDs 4LDE, 4LDO, and 6MXT). It has been proposed based on the crystal structures and mutagenesis studies that these polar interactions are critical for inducing a contracted conformational change of the ligand-binding pocket that leads to receptor activation (Rasmussen et al., 2011a; Masureel et al., 2018). Different $\beta_{2}$-agonists with distinct head groups may engage in slightly different polar interaction networks with the receptor, resulting in their different receptor activation efficacies (Rasmussen et al., 2011a; Ring et al., 2013; Masureel et al., 2018). Salmeterol and salbutamol are both partial agonists, and they share the same saligenin head group, which compared with the catechol group has one additional methylene between the meta-hydroxyl group and the phenyl ring. In the crystal structures, the two hydroxyl groups in the catechol group of epinephrine or in the saligenin group of salmeterol interact with two serine residues in TM5 through hydrogen bonding (Ring et al., 2013; Masureel et al., 2018). These hydrogen bonds contribute to the inward movement of TM5, which is associated with receptor activation. The additional methylene in the saligenin head group may result in a weaker stabilization of TM5 in the active conformation compared with epinephrine. Results from molecular dynamics (MD) simulation studies also suggested that salmeterol stabilizes a less stable active conformation of $\beta_{2} \mathrm{AR}$ compared with epinephrine because of the long hydroxymethyl group in the saligenin group of salmeterol (Masureel et al., 2018). Therefore, the saligenin group in salmeterol and salbutamol accounts for their partial agonism. In contrast, isoproterenol as a classic full agonist shares the same catechol group as epinephrine.

The synthetic ligand, BI-167107, is a full $\beta_{2}$-agonist and has been used in the structural studies of $\beta_{2} \mathrm{AR}$ (Rasmussen et al., 2011a,b). The head group of BI167107 is distinct from epinephrine or salmeterol, but very similar to the quinoline-2-one group in the ultraLABA, indacaterol, which is also a full $\beta_{2}$-agonist (Sturton et al., 2008) (Fig. 1). The head group of BI167107 contains an amine group at the same position relative to the ethanolamine group as the metahydroxyl group in the catechol group of epinephrine, allowing it to also form a hydrogen bond with residue Ser203 $3^{5.43}$ in TM5 (Fig. 2A) (Supplemental Material 1-PDB ID 4LDE). Consistently, formoterol, another full $\beta_{2}$-agonist, also has an amine group in its head group at the same position as the meta-hydroxyl group in the catechol group of epinephrine that may form a similar hydrogen bond with Ser $203^{5.43}$. Therefore, it is likely that this hydrogen bond is an important structural determinant for the efficacy of some $\beta_{2}$-agonists.

b. Duration of action. The mechanism for the duration of in vivo action of $\beta_{2}$-agonist drugs has attracted much research interest since the early development of 


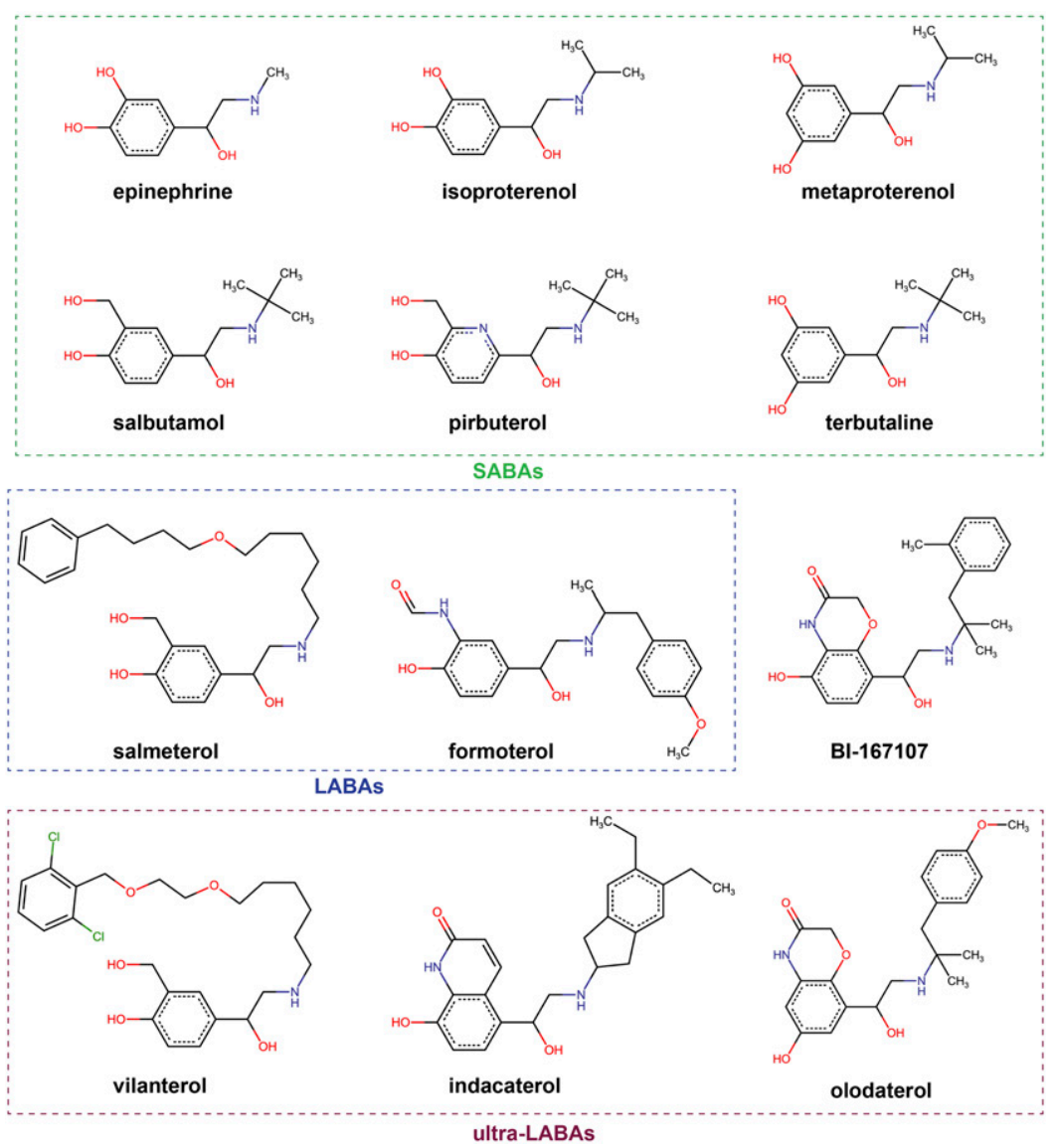

Fig. 1. Chemical structures of $\beta_{2}$-agonists. SABAs, LABAs, and ultra-LABAs are indicated by boxes with green, blue, and red colors, respectively.

$\beta_{2}$-agonists. In general, there are many factors that determine the duration of drug action, including the binding and rebinding to the target and the plasma halflife (Vauquelin and Charlton, 2010). The first synthetic agonist isoproterenol shares the same pharmacophore as epinephrine, the catechol group, which can be rapidly metabolized by catechol- $O$-methyl transferase. Therefore, initial efforts in the development of long-lasting $\beta_{2^{-}}$ agonist compounds were directed at modifying the catechol group. This led to the discovery of salbutamol and terbutaline with noncatechol pharmacophores that are resistant to catechol- $O$-methyl transferase metabolism and demonstrate a prolonged duration of action compared with isoproterenol (Waldeck, 2002), even though both drugs are considered as SABAs.

Early pharmacological studies on salmeterol and formoterol as the two classic LABAs provided several possible mechanisms to explain their prolonged action (Coleman, 2009; Szczuka et al., 2009). The diffusion microkinetic model proposed by Anderson et al. (1994) suggested that the high lipophilicity of these two ligands allows them to be able to incorporate into the lipid bilayers in the vicinity of the receptor, and thus the cell membrane acts as a depot and maintains a high local concentration of the drugs. The microkinetic model well explained an interesting pharmacological property of lipophilic LABAs known as the reassertion of relaxation (Naline et al., 1994; Anderson et al., 1996; Bergendal et al., 1996). It has been shown by in vitro studies that $\beta_{2} \mathrm{AR}$ antagonists can reverse the relaxation of airway tissues by salmeterol and formoterol, but that subsequent washout of all ligands leads to a reappearance of tissue relaxation even when no additional agonist was added. The microkinetic model also explained the concentration-dependent duration of action of formoterol in the washout experiments (Szczuka et al., 2009). However, the duration of action of salmeterol is concentration-independent, and ligand-binding assays suggested a persistent binding of salmeterol to the receptor (Nials et al., 1993), which cannot be adequately explained by the microkinetic mechanism. To explain the action of salmeterol, Coleman et al. (1996) proposed another model, the exosite model, suggesting the existence of an exosite in addition to the orthosteric active site in $\beta 2 A R$. It is postulated that the exosite accommodates the aryloxyalkyl tail group of salmeterol and acts as an anchoring region to keep the ligand in the vicinity of $\beta_{2} \mathrm{AR}$. This model relies on the specific structural characteristic of the aryloxyalkyl tail of salmeterol and provides a favorable interpretation of the unique pharmacological properties of this drug. However, the nature 

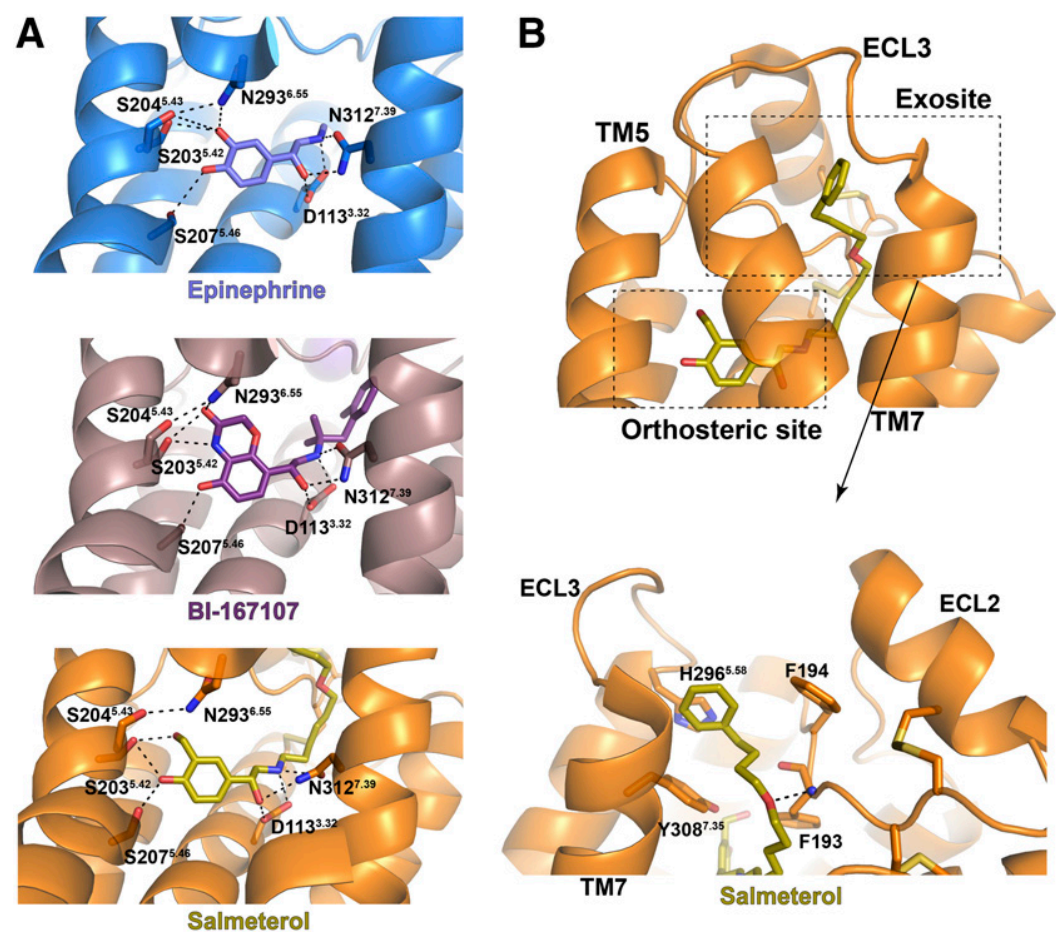

Fig. 2. Binding sites for $\beta_{2}$-agonists revealed by crystal structures. (A) Crystal structures of human $\beta_{2} \mathrm{AR}$ in complex with epinephrine (Supplemental Material 1-PDB ID 4LDO), BI-167107 (Supplemental Material 1-PDB ID 4LDE), and salmeterol (Supplemental Material 1-PDB ID 6MXT). Polar interactions are shown as dashed lines. Residues in $\beta_{2} \mathrm{AR}$ that interact with each ligand are shown as sticks. (B) Exosite for the binding of the tail group of salmeterol in the crystal structure of human $\beta_{2} \mathrm{AR}$ with salmeterol (Supplemental Material 1-PDB ID 6MXT).

and the location of the exosite have been the subject of debate.

The recently published crystal structure of $\beta_{2} \mathrm{AR}$ bound to salmeterol clearly revealed the molecular details of the exosite that is located in the extracellular vestibule of $\beta_{2} \mathrm{AR}$ formed by residues from the extracellular ends of TM6 and TM7 and the extracellular loop (ECL)2 (Masureel et al., 2018) (Fig. 2B) (Supplemental Material 1-PDB ID 6MXT). The ether oxygen atom in the aryloxyalkyl tail of salmeterol forms a hydrogen bond with the main chain amine group of Phe193. It serves as a hinge point where the tail of salmeterol bends to allow the phenyl group at the end of the tail to fit the exosite and form aromatic interactions with the surrounding residues Phe194, Tyr308 ${ }^{7.35}$, and His $296^{6.58}$. The position of this ether oxygen has been proven to be important for the binding of salmeterol (Isogaya et al., 1998). Salbutamol, which shares the same saligenin head group as salmeterol, but lacks the long tail, has an almost 1000-fold lower affinity compared with salmeterol with a short-acting property (Baker, 2010). Therefore, the binding events in the exosite are important for the high-affinity and longlasting action of salmeterol. Previous mutagenesis studies also suggest that exosite mutations could greatly reduce the affinity of salmeterol, but had little effect on the affinity of salbutamol (Baker et al., 2015). Such a binding mode also suggests a bivalent nature of salmeterol. It has been proposed that multivalent ligands that can occupy multiple physically linked sites simultaneously may gain markedly increased affinity and extended receptor residence time through a forced proximity mechanism (Vauquelin and Charlton, 2010, 2013; Vauquelin et al., 2015), which most likely applies to salmeterol. The association of one moiety of salmeterol with the receptor, for example, the binding of the tail group in the exosite, forces the other moiety to be in a proximity of its target site to increase the propensity of rebinding, resulting in a prolonged receptor residence time.

Noticeably, one ultra-LABA, vilanterol, is a derivative of salmeterol that contains an additional ether bond and a few other minor modifications (Fig. 1). If vilanterol adopts a similar binding pose as salmeterol, it is possible that this additional ether group may also hydrogen bond with the receptor, providing more structural restrictions to the flexible aryloxyalkyl tail to enhance the binding of this tail in the exosite. This may explain the longer duration of action of vilanterol compared with salmeterol (Slack et al., 2013). Other ultra-LABAs such as olodaterol and indacaterol do not contain long tail groups, but share a high chemical similarity with BI-167107, the $\beta_{2}$-agonist used in several structural studies of $\beta_{2} \mathrm{AR}$. BI-167107 is not a drug, but it has been shown to exhibit an extremely slow dissociation rate (Rasmussen et al., 2011a). Compared with the catechol group in epinephrine, the hydroxylquinoline ring head group of BI-167107 forms a more extensive aromatic interaction network with surrounding aromatic residues as well as additional 
hydrogen bonds between the ether oxygen of the quinolone ring and side chains of residues Asn293 ${ }^{6.55}$ and Tyr30 $8^{7.35}$. In addition, the methylbenzene ring tail group of BI-167107 forms hydrophobic and aromatic interactions with residues His93 $3^{2.64}$, W109 $9^{3.28}$, and Ile309 ${ }^{7.36}$. Those additional interactions contribute to the high affinity and slow dissociation rate of BI-167107. A similar structural mechanism may also explain the long-lasting action of olodaterol and indacaterol. These two compounds contain large aromatic head groups such as the quinoline-2-one group in indacaterol and additional aromatic tail groups that can engage in more extensive interactions with the receptor compared with epinephrine, resulting in a stronger binding to the receptor.

c. Receptor-subtype selectivity. As discussed before, a high selectivity of $\beta_{2}$-agonist over $\beta_{1} \mathrm{AR}$ may help to reduce its cardiac toxicity. Salmeterol is a highly selective $\beta_{2}$-agonist developed in 1980s (Ball et al., 1991) with a $\sim 3000$-fold selectivity for $\beta_{2} \mathrm{AR}$ over $\beta_{1} \mathrm{AR}$ (Baker, 2010). The exosite revealed by the crystal structure that accommodates the long tail group of salmeterol well explains such high selectivity. Two aromatic residues that directly interact with salmeterol in the exosite, F194 and H296 $6^{6.58}$ (Fig. 2B) (Supplemental Material 1-PDB ID 6MXT), are replaced by two nonaromatic residues N313 and V202 in the $\beta_{1} \mathrm{AR}$, which would significantly compromise salmeterol binding to $\beta_{1} \mathrm{AR}$. The sampling of the nonconserved region, the exosite, by salmeterol results in its high selectivity. Also consistent with the proposed mechanism is the decreased selectivity of salbutamol (20-fold for $\beta_{2} \mathrm{AR}$ ), which lacks the tail group required for exosite interaction.

$\beta_{1} \mathrm{AR}$ and $\beta_{2} \mathrm{AR}$ share a very high sequence similarity (Masureel et al., 2018). Specifically, their orthosteric ligand-binding pockets are structurally very similar to each other, which is expected, as both receptors recognize the same endogenous ligands. Therefore, ligands that primarily occupy the conserved orthosteric binding pockets usually do not have high receptor-subtype selectivity. The same problem is well studied for another GPCR family, the muscarinic acetylcholine receptor family with five members, M1_5 receptors (M1-5R), which will be discussed in details in the next section. Because they all recognize the same endogenous ligand, they share a highly similar orthosteric site. Traditional muscarinic antagonists targeting the orthosteric sites are generally associated with low selectivity and severe side effects (Kruse et al., 2014b). However, there are well-characterized allosteric ligandbinding sites in these receptors that are structurally distinct from the conserved orthosteric sites. These allosteric sites are divergent among all five muscarinic acetylcholine receptors, which offer great opportunities for designing allosteric modulators for each muscarinic acetylcholine receptor that can achieve high receptorsubtype selectivity (Digby et al., 2010). Interestingly, the exosite in $\beta_{2} \mathrm{AR}$ is reminiscent of the allosteric site in M2R revealed by crystal structures (Masureel et al., 2018). As an old drug that was developed more than three decades ago, salmeterol may belong to a new type of GPCR-targeting drug that started emerging recently, the bitopic drugs, which can occupy both the orthosteric and allosteric sites (Kamal and Jockers, 2009). These drugs may be designed rationally with sufficient structural information to gain desired pharmacological properties.

$d$. Biased signaling. The first structural insights into the biased signaling of $\beta_{2} \mathrm{AR}$ came from NMR studies using a ${ }^{19} \mathrm{~F}$-labeled receptor in the presence of a number of ligands, including two $\beta$-arrestin-biased ligands, carvedilol and isoetharine (Liu et al., 2012). The results suggested that the tail groups of these two ligands might be the structural determinants for their biased signaling properties, which directly interact with the extracellular part of TM7. In the experiments, carvedilol and isoetharine affected the conformational equilibrium of TM7 in different ways compared with nonbiased $\beta_{2} \mathrm{AR}$ ligands. The conformation of TM7 is believed to play an important role in the functional selectivity of $\beta_{2} \mathrm{AR}$.

However, data from the structural and site-directed mutagenesis studies, investigating salmeterol action, suggested that the interactions between the ligand head group and the receptor affect the biased signaling properties of salmeterol (Masureel et al., 2018). In the structure of $\beta_{2} \mathrm{AR}$ bound to epinephrine (Supplemental Material 1-PDB ID 4LDO), the two hydroxyl groups in the catechol head group of epinephrine are involved in a polar interaction network with residues Ser204 $4^{5.43}$ and Asn293 ${ }^{6.55}$. Mutations of these two residues could significantly compromise $\beta$-arrestin recruitment by the full agonist isoproterenol, but had more moderate effects on the $\mathrm{G}_{\mathrm{s}}$ activation. In the structure of $\beta_{2} \mathrm{AR}$ bound to salmeterol (Supplemental Material 1-PDB ID 6MXT), Ser204 $4^{5.43}$ and Asn293 3.55 are not involved in the direct polar interactions with the saligenin group of salmeterol, and mutations of these two residues also had moderate effects on the $G_{s}$ activation. It was proposed that the lack of direct interaction between the head group of salmeterol and Asn293 $3^{6.55}$ might account for the weak ability of salmeterol in recruiting $\beta$-arrestins. This mechanism may also explain the biased signaling property of salbutamol that shares the same saligenin head group with salmeterol (van der Westhuizen et al., 2014).

\section{Muscarinic Acetylcholine Receptors and Muscarinic Antagonists}

\section{A. Pathophysiological Roles and Signaling of Muscarinic Receptors in Asthma}

Muscarinic and nicotinic cholinergic receptors mediate metabotropic and ionotropic effects of acetylcholine on the central and peripheral nervous systems 
(Caulfield, 1993). Muscarinic acetylcholine receptors (MRs, or mAChRs), which are more sensitive to muscarine than to nicotine, are a group of class A GPCRs comprising five distinct subtypes, named as muscarinic M1, M2, M3, M4, and M5 receptors (M1R-M5R) (Hammer et al., 1980; Hammer and Giachetti, 1982; Mitchelson, 1988; Dörje et al., 1991; Jones et al., 1991; Caulfield, 1993; Caulfield and Birdsall, 1998; Ishii and Kurachi, 2006). M1R, M3R, and M5R are coupled to the $\mathrm{G}_{\mathrm{q} / 11}$ family of $\mathrm{G}$ proteins, whereas M2R and M4R are coupled to the $\mathrm{G}_{\mathrm{i} / \mathrm{o}}$ family of $\mathrm{G}$ proteins.

MRs are expressed by structural cells in the airways, predominantly ASM, airway epithelium, and airway fibroblasts (Gosens et al., 2006; Profita et al., 2009; Radosa et al., 2011; Wallon et al., 2011; Kistemaker and Gosens, 2015). Acetylcholine binds to airway MRs to trigger smooth muscle contraction and mucus secretion. M2R and M3R have been shown to play major roles in airway physiology, and in diseases such as asthma and COPD (Gross and Skorodin, 1984; Pare et al., 1997; Jeffery, 2001; Rabe et al., 2007; Buels and Fryer, 2012; Kistemaker et al., 2012; Ferkol and Schraufnagel, 2014).

M2R are expressed on ASM and on parasympathetic neurons. Although M2R is the major type of MR expressed on ASM (Roffel et al., 1988; Haddad et al., 1994), it has a very limited role in ASM contraction. M2R can activate the $\mathrm{G} \alpha_{\mathrm{i}}$ subunit, which can then bind to and inhibit adenylyl cyclases activated by $\mathrm{G} \alpha_{\mathrm{s}}$. Thus, M2R activation constrains the signaling and bronchorelaxant effects of $\beta_{2} \mathrm{AR}$ by antagonizing $\beta_{2} \mathrm{AR} / \mathrm{G}_{\mathrm{s}}$ activation of adenylyl cyclases (Kume and Kotlikoff, 1991; Fernandes et al., 1992; Schramm et al., 1995). However, M2R expressed presynaptically on the parasympathetic nerve endings, when activated, exerts negative feedback on neuronal acetylcholine release, thereby limiting bronchoconstriction. The dysfunction of the presynaptic M2R has been proposed as a pathophysiological mechanism of AHR in asthma (Fryer and Jacoby, 1998).

M3R are the dominant receptor subtype for bronchial smooth muscle contraction, and are found in airway smooth muscle and submucosal glands (Mak and Barnes, 1990; Haddad et al., 1991; Fernandes et al., 1992; Mak et al., 1992; Barnes, 1993; Struckmann et al., 2003; Gosens et al., 2006; Buels and Fryer, 2012). M3R can mediate ASM contraction through both calciumdependent and calcium-independent mechanisms (Harnett and Biancani, 2003; Gosens et al., 2006; Bradley et al., 2016). The calcium-dependent mechanism is centered on Gq-mediated phospholipase C activation. Stimulation of M3R on ASM by acetylcholine initiates a conformational change in the receptor that promotes its association and activation of the heterotrimeric $\mathrm{G}$ protein $\mathrm{G}_{\mathrm{q}}$. The activated $\alpha$ subunit of $\mathrm{Gq}$ in turn activates membrane-bound phospholipase $\mathrm{C}$, which hydrolyzes phosphatidylinositol 4,5-bisphosphate into 1,2-diacylglycerol and inositol 1,4,5-trisphosphate.
Inositol 1,4,5-trisphosphate diffuses into the cytosol and binds its receptor, the inositol triphosphate receptor on the sarcoplasmic reticulum, thereby augmenting the release of $\mathrm{Ca}^{2+}$. The rise in intracellular free $\mathrm{Ca}^{2+}$ next enhances conductivity of neighboring ryanodine receptors, and together they generate a transient $\mathrm{Ca}^{2+}$ wave (Grandordy et al., 1986; Chilvers et al., 1990; Roffel et al., 1990). Increased $\mathrm{Ca}^{2+}$ induces the formation of $\mathrm{Ca}^{2+} /$ calmodulin complexes capable of activating myosin light chain kinase. The subsequent phosphorylation of myosin light chain allows the actin-myosin crossbridge cycle to operate. In parallel, both $\mathrm{Ca}^{2+}$ and 1,2diacylglycerol recruit protein kinase $\mathrm{C}$ (PKC) to the membrane. The activated PKC phosphorylates an inhibitory regulator (named CPI-17) of the downstream myosin-light chain phosphatase (MLCP), promoting the affinity between the CPI-17 and MLCP. MLCP inhibition enhances $\mathrm{Ca}^{2+}$ sensitivity of the system. In contrast, the mechanism by which the M3R mediates calcium-independent ASM contraction is referred to calcium sensitization (Billington and Penn, 2002) via activation of the small GTPase RhoA that in turn activates Rho-kinase. RhoA and Rho-kinase augment agonist-induced contraction primarily by inactivating MLCP. MLCP is inhibited by the direct phosphorylation of its regulatory myosin-binding subunit by Rho-kinase and by binding to CPI-17, which is targeted for phosphorylation by both Rho-kinase and PKC, resulting in higher levels of phosphorylated myosin and smooth muscle contraction (Gosens et al., 2006).

\section{B. Currently Used Muscarinic Antagonist Drugs and Their Pharmacological Properties}

Numerous anticholinergic compounds derived from plant alkaloids (Datura stramonium and Atropa belladonna) were used historically, long before $\beta$-agonists, for the treatment of asthma symptoms (Miraldi et al., 2001; Jackson, 2010). Atropine, along with hyoscyamine and scopolamine, were some of the anticholinergic compounds first isolated from plant alkaloids in 1833 . Its bronchodilator properties were first used for asthma from the late 18th century. Atropine and other anticholinergic agents exert their bronchodilator effects through the blockade of MRs in the airways. As a tertiary ammonium derivative, atropine is a nonselective antagonist with similar affinity for all of the MR subtypes (Moulton and Fryer, 2011; Buels and Fryer, 2012). The half-life of atropine for M3R residence is 3.5 hours. Although extensively used in the past, atropine is rarely used at the present time because it is well absorbed into the systemic circulation and penetrates the blood-brain barrier, leading to multiple systemic side effects, including tachycardia (Donald et al., 1967; Smirnov, 1989; Cazzola et al., 2012).

In the 1970s, new anticholinergic medications were developed, given the need to develop alternatives to $\beta_{2^{-}}$ agonist therapies. The newer anticholinergic agents are 
water-soluble, quaternary ammonium compounds that are poorly absorbed, and, when administered by inhalation, cause fewer systemic side effects (Gross, 2006). Among them, ipratropium bromide and oxitropium bromide are short-acting muscarinic antagonist (SAMA) drugs that have been used in the treatment of respiratory diseases. Like atropine, they are also nonselective anticholinergics, blocking both the prejunctional M2R and postjunctional M3R. The SAMA agent ipratropium bromide is a synthetic quaternary ammonium compound that is chemically related to atropine. Its half-life for M3R residence is 3.2 hours. Oxitropium bromide is based on the scopolamine, and its half-life is slightly longer.

Several long-acting muscarinic antagonists (LAMAs) are under investigation or are available for the treatment of obstructive airway diseases. Tiotropium bromide is also a quaternary ammonium derivative compound with two thiophene rings, displaying a much higher affinity (6- to 20-fold) for MRs compared with ipratropium bromide (Haddad et al., 1994). It has a similar affinity for the M2R and M3R; however, unlike ipratropium, tiotropium is functionally selective for the M3R because of its longer pharmacological half-life for the M3R (35 hours vs. 3.6 hours for M2R) (Disse et al., 1993). Initially indicated for the treatment of COPD (Casaburi et al., 2002), tiotropium bromide (Respimat) received Food and Drug Administration approval in 2017 for the use in children with asthma aged 6 years or over (Gosens and Gross, 2018). In fact, tiotropium is the only LAMA that has been studied extensively in asthma, and is the first agent in the class to be indicated as add-on therapy for patients with asthma who are currently treated with maintenance ICS only or ICS and LABA therapy.

In addition to tiotropium, three other LAMAs (aclidinium, umeclidinium, and glycopyrrolate) used in the treatment of COPD have the potential to be developed as therapies for asthma. Two of these LAMAs (umeclidinium and glycopyrrolate) are currently being investigated in clinical trials for asthma (Gilman et al., 1990; Lee et al., 2015; Busse et al., 2016; Ferrando et al., 2017). Aclidinium is similar to tiotropium with a quaternary ammonium group and two thiophene rings, and, although it has a similar affinity for M2R and M3R, it is also functionally selective for M3R based on its extended half-life of 29 hours (Gavaldà et al., 2009; Moulton and Fryer, 2011). Aclidinium is very rapidly hydrolyzed in plasma into its two inactive metabolites (plasma half-life $=2.4$ minutes), thus minimizing systemic side effects. Glycopyrrolate is a M3R selective antagonist with 3-5 times higher affinity for the M3R compared with the M2R (Gavaldà et al., 2014). Its half-life was reported to be 3.7 hours (Haddad et al., 1999; Moulton and Fryer, 2011). The newest LAMA is umeclidinium bromide, which also has a greater affinity M3R based on its slower dissociation from M3R compared with the M2R (82 vs. 9 minutes) (Salmon et al., 2013).

LAMAs are considered to be safe drugs at recommended dosages. However, because MRs are expressed not only in the lungs, but also in the heart and the digestive and urinary tracts, the blockade of different MR subtypes in these organs by LAMA treatment can cause diverse, unwanted physiologic effects. For example, these agents can initially block prejunctional M2R on cholinergic airway nerves that normally reduce the release of the bronchoconstricting neurotransmitter acetylcholine, thus resulting in cough and paradoxical bronchoconstriction (Mann et al., 1984; Lee et al., 2008; Singh et al., 2008; Cazzola et al., 2012). Side effects including cardiovascular morbidity and mortality of inhaled LAMA agents in asthma need to be further studied and defined.

The combination therapy of ICS and bronchodilator has been a mainstay for asthma management for nearly 50 years. However, there are limited options for those who continue to have asthma exacerbations while taking combination ICS/LABA treatment. Thus, the addition of anticholinergics as an add-on therapy to the current regimes may provide alternative treatments to those who are unresponsive on their current treatments or for those who are refractory to $\beta_{2}$-agonists. There are still safety concerns regarding the continued use of $\beta_{2^{-}}$ agonists, especially for those with the single-nucleotide polymorphisms in the $\beta_{2}$-adrenergic receptor gene ADRB2, or for children where reduced bone density and growth are a concern (Restrepo, 2007; Ortega et al., 2014; Pandya et al., 2014). To date, the benefits seen with tiotropium as an add-on therapy in the subgroup of asthmatics with poorly controlled symptomatic asthma also suggest that a broad range of patients with asthma may benefit from anticholinergic therapy regardless of their respective baseline characteristics. Continued research into the mechanism of acetylcholine signaling in asthma and the results of ongoing clinical studies will promote the broader use of anticholinergics as add-on or stand-alone therapies for the management of asthma symptoms.

\section{Structural Insights into Drug Action}

1. Chemical Structures of Clinically Used Muscarinic Antagonists. Almost all of muscarinic antagonist drugs share a similar chemical scaffold as atropine, characterized by an ester between an aromatic ring derivative and a modified tropane or quinuclidine moiety containing a positively charged quaternary ammonium group (Fig. 3).

2. Structural Insights into the Pharmacological Properties of Clinically Used Muscarinic Antagonists. Since the first high-resolution structure of a MR was solved by X-ray crystallography in 2012 (Haga et al., 2012), 15 structures have been deposited in PDB for M1R, M3R, and M4R at inactive antagonist-bound 


\section{A SAMA}

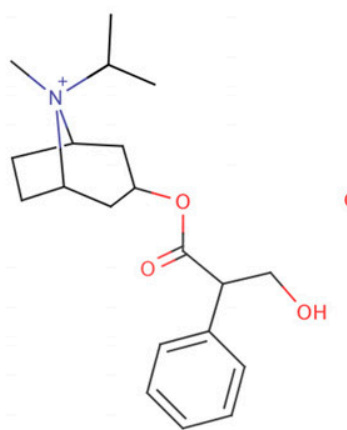

Ipratropium

B

\section{LAMA}
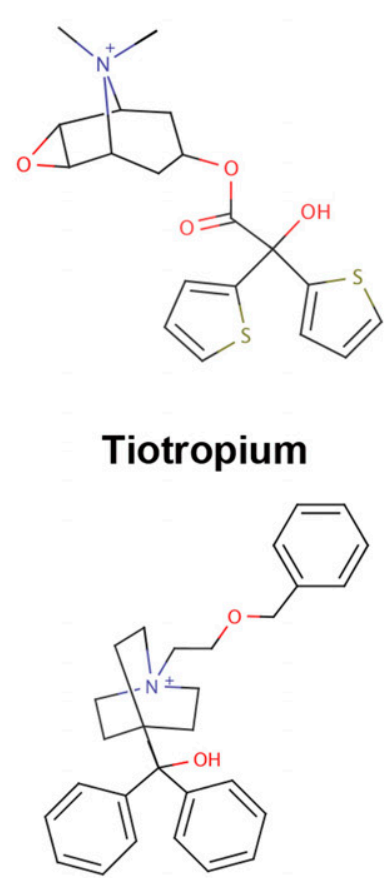

Umeclidinium
Tiotropium
Oxitropium

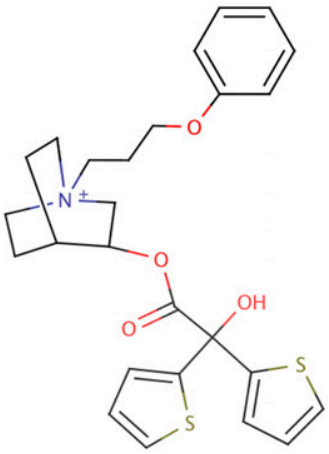

Aclidinium

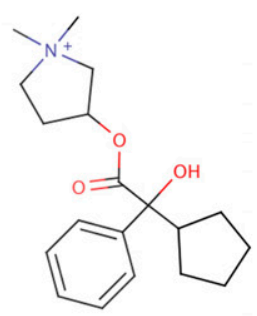

Glycopyrrolate

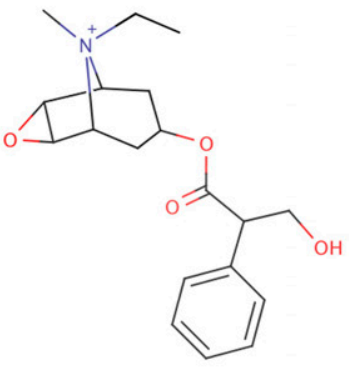

Fig. 3. Chemical structures of currently used muscarinic antagonist drugs. (A) Ipratropium and oxitropium as SAMAs. (B) Tiotropium, aclidinium, umeclidinium, and glycopyrrolate as LAMAs.

states (Kruse et al., 2012; Thorsen et al., 2014; Thal et al., 2016; Liu et al., 2018), and for M2R at both inactive and active states (Table 3) (Haga et al., 2012; Kruse et al., 2013a; Suno et al., 2018). The overall transmembrane structure is similar to those of rhodopsin (Palczewski et al., 2000) and $\beta_{2} \mathrm{AR}$ (Rosenbaum et al., 2007), which has a conserved disulfide bond between $\mathrm{Cys}^{3.25}$ at the $\mathrm{N}$ terminus of TM3 and another Cys residue in the ECL2. MR also has an additional disulfide bond between two Cys residues in the ECL3. The orthosteric binding pocket is formed by amino acids that are identical in all five MR subtypes. A layer of tyrosine residues forms an aromatic cap restricting dissociation of the bound ligand. An allosteric ligandbinding site has been identified at the entrance to the binding pocket near this aromatic cap.

a. Ligand efficacy. Over the past decade, the M1R$\mathrm{M} 4 \mathrm{R}$ were crystallized, and their structures were solved with antagonists such as tiotropium, 3-quinuclidinyl benzilate (QNB), and N-methyl scopolamine (NMS). These ligands have similar chemical structures and bind in similar positions in the orthosteric site (Fig. 4A) (Supplemental Material 1-PDB IDs 3UON, 4DAJ, $5 \mathrm{CXV}$, and 5DSG), which is also the binding site for the endogenous agonist acetylcholine. This orthosteric site is buried within the 7TM core. Strikingly, amino acid sidechains on TM3, 4, 5, 6, and 7 that surround these ligands are fully conserved among all five MR subtypes and are located in similar positions in the orthosteric site across the M1R-M4R structures. The natural agonist acetylcholine is much smaller than these antagonists. Bulky antagonists capable of blocking activation-related contraction of the pocket would be very efficient in locking MRs in an inactive conformation.

M1R, M3R, and M4R have been crystallized in complex with tiotropium, the potent, nonsubtypeselective antagonist (Fig. 4A). Like atropine and other conventional muscarinic antagonists, tiotropium can be considered an inverse agonist because it is able to inhibit both basal and ligand-independent MR signaling (Casarosa et al., 2010). The ligand is almost completely occluded from solvent by a lid comprised of three conserved tyrosines, $\mathrm{Tyr}^{3.33}, \mathrm{Tyr}^{6.51}$, and $\mathrm{Tyr}^{7.39}$. Besides the extensive hydrophobic contacts with the receptor, the bound tiotropium also forms a pair of H-bonds between the ligand hydroxyl/ketone and the conserved Asn ${ }^{6.52}$, as well as charge-charge interactions between the ligand cationic amine and the conserved $\mathrm{Asp}^{3.32}$. The charge-charge interactions involving $\mathrm{Asp}^{3.32}$ are seen in all biogenic amine receptor structures solved to date and have been shown to make a major energetic contribution to binding (Kruse et al., 2013b). The hydrogen-bonding interactions involving $\mathrm{Asn}^{6.52}$ seem to be a unique feature for the MR. This feature was suggested to be an important factor in slowing down ligand dissociation (Tautermann et al., 2013). These interactions also explain the extremely high affinity of tiotropium for M3R $(\mathrm{Ki}=0.010 \mathrm{nM})$ and all other MR subtypes (e.g., $\mathrm{Ki}=0.020 \mathrm{nM}$ for M2R) (Disse et al., 1993; Casarosa et al., 2009; Buels and Fryer, 2012).

As noted above, the orthosteric binding pocket is highly conserved among all MR subtypes. Nevertheless, MR structures display a unique feature-a rather large solvent-accessible vestibule facing the extracellular space (termed extracellular vestibule) that is separated from the orthosteric site by the tyrosine lid. Rearrangement of the tyrosine lid and possibly the ECLs is required to enable tiotropium to exit from the 
TABLE 3

Structures of human M1, M2, and M4 receptors, and rat M3 receptor

\begin{tabular}{|c|c|c|c|c|c|c|}
\hline Receptor & PDB ID & Ligand & Efficacy & Fusion Partner & Resolution $(\AA)$ & References \\
\hline M1R & $5 \mathrm{CXV}$ & Tiotropium & Inverse agonist & $\mathrm{T} 4$ lysozyme (T4L) in ICL3 & 2.70 & Thal et al., 2016 \\
\hline M2R & $3 \mathrm{UON}$ & QNB & Inverse agonist & T4L in ICL3 & 3.00 & Haga et al., 2012 \\
\hline M2R & 4MQS & Iperoxo & Full agonist & None & 3.50 & Kruse et al., 2013a \\
\hline M2R (S110R) & $5 \mathrm{ZKC}$ & NMS & Inverse agonist & BRIL in ICL3 & 2.30 & Suno et al., 2018 \\
\hline M2R (S110R) & $5 \mathrm{ZKB}$ & AF-DX 384 & Antagonist & BRIL in ICL3 & 2.95 & Suno et al., 2018 \\
\hline M2R (S110R) & $5 Z \mathrm{ZK} 3$ & QNB & Inverse agonist & BRIL in ICL3 & 2.60 & Suno et al., 2018 \\
\hline M3R & $4 \mathrm{U} 15$ & Tiotropium & Inverse agonist & Minimal T4L (mT4L) in ICL3 & 2.80 & Thorsen et al., 2014 \\
\hline M3R & $4 \mathrm{U} 16$ & NMS & Inverse agonist & $\mathrm{mT} 4 \mathrm{~L}$ in ICL3 & 3.70 & Thorsen et al., 2014 \\
\hline M3R & $5 \mathrm{ZHP}$ & Compound 6o (BS46) & Antagonist & $\mathrm{mT} 4 \mathrm{~L}$ in ICL3 & 3.10 & Liu et al., 2018 \\
\hline M4R & $5 \mathrm{DSG}$ & Tiotropium & Inverse agonist & $\mathrm{mT} 4 \mathrm{~L}$ in ICL3 & 2.60 & Thal et al., 2016 \\
\hline
\end{tabular}

orthosteric binding pocket. In comparison with the highly conserved orthosteric site, the extracellular vestibule is least conserved across the muscarinic subtypes, showing multiple sequence differences. In the MD simulation studies of M2R and M3Rs with tiotropium, the ECLs, especially the ECL2, appeared to be more flexible in the M2R as compared with the M3R (Kruse et al., 2012), suggesting a higher energy barrier to be overcome for tiotropium to exit from the M3R. The observations from MD simulations are consistent with mutagenesis data and data from dissociation experiments of tiotropium, which suggested that the more

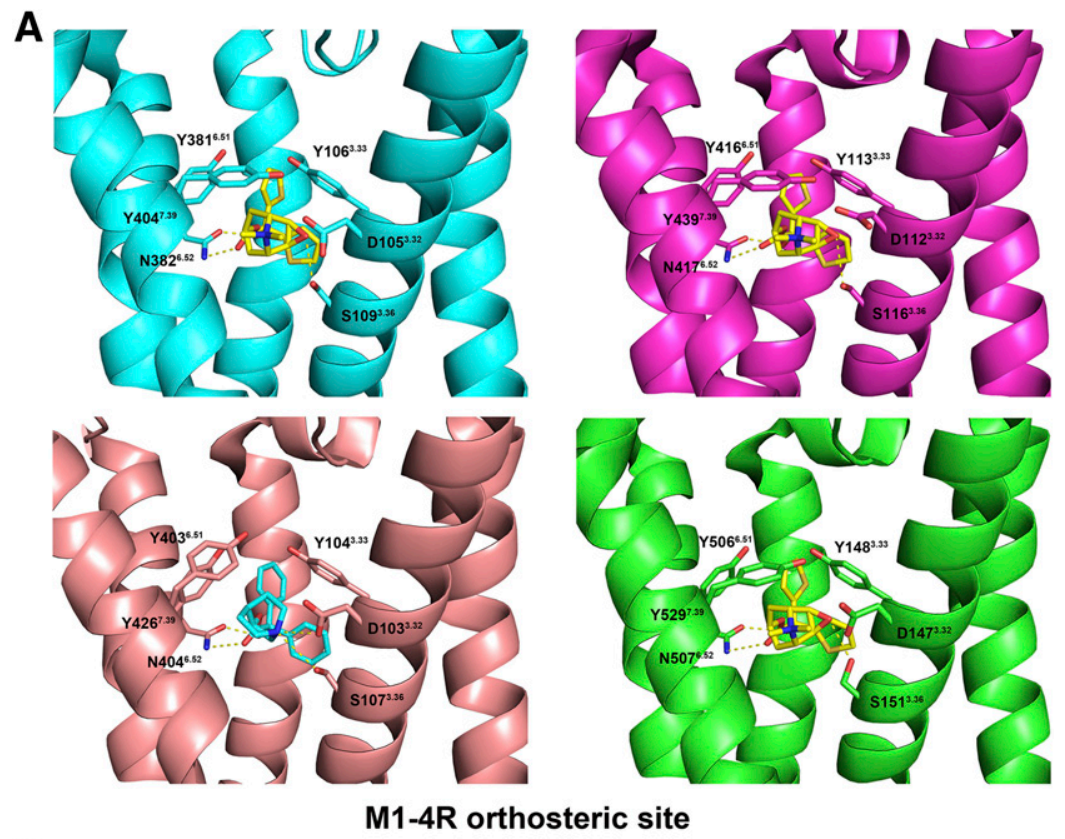

B
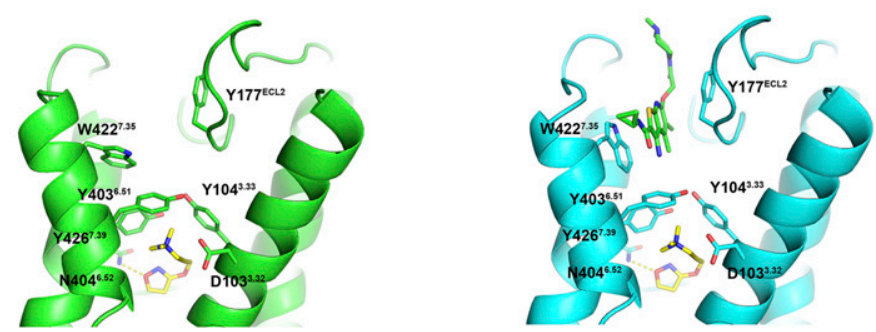

\section{M2R allosteric site}

Fig. 4. Binding sites in MR crystal structures. (A) Orthosteric sites for tiotropium in the crystal structures of tiotropium-bound M1R (top left, Supplemental Material 1-PDB ID 5CXV), M3R (bottom right, Supplemental Material 1-PDB ID 4DAJ), and M4R (top right, Supplemental Material 1-PDB ID 5DSG), and the orthosteric site for QNB in the crystal structure of M2R (bottom left, Supplemental Material 1-PDB ID 3UON). Polar interactions are shown as dashed lines. (B) Crystal structure of active M2R with the orthosteric agonist iperoxo only (left, Supplemental Material 1-PDB ID 4MQS, allosteric site empty) and crystal structure of active M2R with iperoxo and a PAM named LY2119620 (right, Supplemental Material 1-PDB ID 4MQT). Full agonist iperoxo is in the orthosteric site of both structures. 
flexible loop region in the M2R in combination with the lack of residue Lys ${ }^{7.32}$ causes the kinetic selectivity of the M3R over the M2R (Tautermann et al., 2013). These structural observations explain tiotropium's clinically important kinetic selectivity, long duration of action, and minimal non-M3R-mediated side effects.

b. Receptor subtype selectivity in the orthosteric site. Although residues lining the orthosteric site in the MR family are absolutely conserved, this high degree of sequence conservation does not preclude the existence of differences in the three-dimensional architecture of the orthosteric site between the different receptor subtypes (Fig. 4A). In the M4R structure (Supplemental Material 1-PDB ID 5DSG), $\mathrm{Asn}^{3.32}$ has its rotamer point away from tiotropium and forms a network of $\mathrm{H}$-bond interactions with neighboring residues such as $\mathrm{Tyr}^{7.39}$ and $\mathrm{Tyr}^{7.43}$, which is distinct from the M1R, M2R, and M3R orthosteric sites. The tiotropium-bound M1R, M3R, and M4R and QNBbound M2R structures also show considerable differences in the conformations of residues $\mathrm{Asn}^{3.32}$ Tyr $^{7.39}$, and $\mathrm{Tyr}^{7.43}$ Such differences contribute to the marked difference in the potency for pirenzepine, which serves as a canonical orthosteric antagonist of MRs, with a rank order potency of M1R $>$ M4R $>$ M3R $>$ M2R (Caulfield and Birdsall, 1998; Thal et al., 2016). This suggests that ligand selectivity can be achieved through subtle differences in the receptor conformational dynamics.

One such structural difference near the orthosteric site in M2R and M3R results from the replacement of Phe181 in the ECL2 of the M2R with Leu225 in M3R, which interacts with one aromatic ring of the bound tiotropium or QNB. This creates a pocket in the M3R not found in the M2R. This single amino acid difference was exploited in the development of subtype-selective ligands. Using molecular docking and structure-based design strategy, Liu et al. (2018) discovered a M3Rselective antagonist that showed up to 100 -fold selectivity over the M2R in in vitro assays and over 1000-fold selectivity when tested in vivo.

Recently, a M2R structure with a mutation S110R was solved in complex with a M2R/M4R-selective antagonist AF-DX 384 (Suno et al., 2018) (Supplemental Material 1-PDB ID 5ZKB). This ligand has a higher affinity for M2R/M4R than for M1R/M3R. In other M2R structures with QNB, the orthosteric ligand is almost completely occluded from the solvent with the tyrosine lid located directly above the ligand (Haga et al., 2012). By contrast, in the AF-DX 384-bound S110R M2R structure, the tyrosine lid opens up and the orthosteric site becomes more accessible from the extracellular space, thus allowing the two propyl groups of AF-DX 384 to extend from the orthosteric pocket toward the extracellular surface. Because the residues forming contacts with AF-DX 384 are completely conserved among MRs, the residues responsible for subtype selectivity cannot be identified based on the crystal structure alone. In MD simulations of the wild-type M2R and M3R with AF-DX 384, ligand-receptor contacts were found to be tighter with a few residues in TM2, TM3, and TM6 in the M2R. This observation suggested that the subtype selectivity of AF-DX 384 may be a consequence of not only the static interaction seen in the crystal structure, but also the protein dynamics-tightening of the ligand-receptor contacts in the M2R compared with the M3R.

c. The structural basis for the action of allosteric modulators as potential novel asthma drugs. Before crystal structures were solved for MRs, evidence from functional assays proved the existence of allosteric modulators of these receptors (Lüllmann et al., 1969; Clark and Mitchelson, 1976). Some modulators such as gallamine and alcuronium were reported to interact with MRs, but not to compete with orthosteric ligands such as acetylcholine (Stockton et al., 1983; Proska and Tucek, 1994). These modulators limit or enhance the binding of orthosteric ligands and also slow their dissociation, resulting in slowed binding kinetics. Extensive studies of these modulators strongly suggested a common allosteric site on all five MRs (Gregory et al., 2007). Mutagenesis experiments further implicated contributions from ECL2, ECL3, and the top of TM7 to this common allosteric site (Leppik et al., 1994; Krejcí and Tucek, 2001; Buller et al., 2002; Jakubík et al., 2005). Multiple novel MR ligands with high subtype selectivity have been predicted to target this allosteric site (Digby et al., 2010; Keov et al., 2011) or occupy both allosteric and orthosteric sites simultaneously (bitopic ligands) (Bock and Mohr, 2013; Lane et al., 2013).

This predicted common allosteric site was confirmed and clearly illustrated in the solved crystal structures of M1R-M4R (Table 3). This allosteric site, termed the extracellular vestibule, is lined by residues that have been previously implicated in the binding of allosteric modulators. It is located directly above (extracellular to) the orthosteric binding site, also consistent with the observation that many muscarinic allosteric modulators can slow dissociation of orthosteric ligands (Kruse et al., 2014a). In comparison with the highly conserved orthosteric site, the extracellular vestibule is less conserved in sequence across the muscarinic subtypes, for example, M2R and M3R can differ by up to 11 substitutions among the 24 residues around this allosteric site (Korczynska et al., 2018). The divergence in amino-acid composition leads to distinct differences in both the shape and charge distribution of the allosteric site, and likely the dynamics of the ECL regions. These observations from MR structures can explain why some of the best-studied MR allosteric modulators such as gallamine are cationic compounds (Thal et al., 2016). A segment of acidic amino acids, EDGE, in the ECL2 of the M2R, has been found important for the higher binding potency of gallamine 
for the M2R compared with the other four MR (Gnagey et al., 1999).

In comparison with the large extracellular vestibule in the inactivate MR structures, this outer cavity in the M2R undergoes a pronounced contraction upon agonist/ nanobody-triggered activation, primarily due to the inward movement of the extracellular portion of TM6 (Kruse et al., 2013a) (Fig. 4B) (Supplemental Material 1-PDB ID 4MQT). Importantly, one of the agonistbound active M2R structures was solved in complex with LY2119620, a positive allosteric modulator (PAM), providing the first structural view of how a drug-like allosteric ligand binds to a GPCR. In this complex structure, the allosteric modulator is located directly above the orthosteric agonist. The contraction in the extracellular vestibule results in a narrower allosteric site and a smaller orthosteric site. The much smaller size of the orthosteric site is reflected by the lower molecular weight of muscarinic agonists compared with antagonists and inverse agonists (Kruse et al., $2014 a, b)$. The agonist-stabilized contraction of the extracellular vestibule enables the PAM to engage in extensive interactions with this outer receptor cavity, which include aromatic stacking, H-bonding, and charge-charge interactions. In particular, the aromatic rings of the PAM are situated directly between Tyr177 in ECL2 and Trp422 $2^{7.35}$, forming a threelayered aromatic stack. Interestingly, the two active structures of the M2R are highly similar irrespective of whether the PAM is bound, suggesting that the allosteric site conformation is largely preformed after the binding of the orthosteric agonist (Fig. 4B) (Kruse et al., 2013a). These findings support the concept that muscarinic PAMs enhance the receptor affinity of orthosteric agonists by stabilizing the active conformation of the receptor and slowing agonist dissociation from the orthosteric site.

These crystal structures of human and rat MRs solved at both inactive and active states, together with MD simulation and mutagenesis studies, provided a solid basis for the development of subtype-selective therapeutics. Specifically, the extracellular vestibule represents an attractive target for the development of subtype-selective allosteric agents for MRs. Recently, a structure-based docking campaign was prosecuted against the allosteric site observed in the antagonistbound inactive structure of M2R, which led to the discovery of a new PAM (Korczynska et al., 2018). It has been shown to enhance the binding of NMS and the drug scopolamine itself. Furthermore, this PAM slowed the dissociation rate of NMS from the M2R by 50-fold, but did not demonstrate similar effects on other MRs (Korczynska et al., 2018). This study supports the feasibility of discovering PAMs that confer subtype selectivity to nonselective orthosteric drugs of MR, which are expected to reduce the off-target effects of nonselective orthosteric drugs in asthma.

\section{Cysteinyl Leukotriene Receptors and Leukotriene Antagonists}

\section{A. Pathophysiological Roles and Signaling of Cysteinyl Leukotriene Receptors in Asthma}

1. Cysteinyl Leukotriene Synthesis. CysLTs are a class of bioactive fatty acids whose formation and signaling are associated with airway inflammation. These endogenous bioactive fatty acids are derived from the $\omega-6$ eicosanoid, arachidonic acid (AA). AA is esterified in phospholipids at the sn2 position, and under conditions of stress it is released by phospholipase $\mathrm{A}_{2}$ and metabolized into bioactive fatty acids primarily through cyclooxygenase to form PGs or lipoxygenase pathways (Funk, 2001; Dennis and Norris, 2015). Leukotrienes are generated by 5-LOX in leukocytes. Specifically, 5-LOX together with 5-LOX-activating protein transforms AA into 5-hydroperoxyeicosatetraenoic acid. The peroxide of 5-hydroperoxyeicosatetraenoic acid spontaneously reduces to form 5-hydroxyeicosatetraenoic acid (5-HETE). 5-HETE is also a substrate for 5-LOX in which the end product is the epoxide ring containing leukotriene $\mathrm{A}_{4}\left(\mathrm{LTA}_{4}\right) . \mathrm{LTA}_{4}$ can be either rapidly hydrolyzed by $\mathrm{LTA}_{4}$ hydrolase to form $\mathrm{LTB}_{4}$ or conjugated with glutathione, a reaction that is catalyzed by $\mathrm{LTC}_{4}$ synthase to generate $\mathrm{LTC}_{4}$ as the first CysLT in this pathway. Once transported out of cells, $\mathrm{LTC}_{4}$ can then be metabolized by extracellular enzymes to form $\mathrm{LTD}_{4}$ and $\mathrm{LTE}_{4}$. Some studies also suggested that certain cells expressing $\mathrm{LTC}_{4}$ synthase can catalyze the conversion of extracellular $\mathrm{LTA}_{4}$ to $\mathrm{LTC}_{4}$, providing another source of CysLTs (Maclouf et al., 1994). LTC 4 , $\mathrm{LTD}_{4}$, and $\mathrm{LTE}_{4}$ are the major bioactive members of the CysLT family, all of which are lipid and peptide hybrid molecules with a cysteine amino acid attached to the $\mathrm{C} 6$ of the fatty acid chain (Fig. 5). In the airways, CysLTs are mainly generated and released from innate immune cells, including eosinophils, basophils, and mast cells (Drazen and Austen, 1987).

2. Pathophysiological Roles and Signaling of Cysteinyl Leukotriene Receptors. A term, slow-reacting substance of anaphylaxis, was introduced in the early 20th century to describe some mediators other than histamine to induce anaphylaxis and result in prolonged contraction of bronchial smooth muscle after allergen challenge (Augstein et al., 1973). Such mediators have been shown to induce symptoms of asthma. Studies from several groups using various biophysical and physiologic approaches later demonstrated that slow-reacting substance of anaphylaxis was actually a mixture of CysLTs (Bach et al., 1977; Morris et al., 1978, 1980; Lewis et al., 1980; Samuelsson, 1983). Further studies in the next decade revealed pivotal roles of CysLTs, especially $\mathrm{LTC}_{4}$ and $\mathrm{LTD}_{4}$, in the pathogenesis of asthma. A major breakthrough during that period was the identification and characterization of two GPCRs, CysLT receptor (CysLTR) ${ }_{1}$ and CysLTR , $_{2}$ 

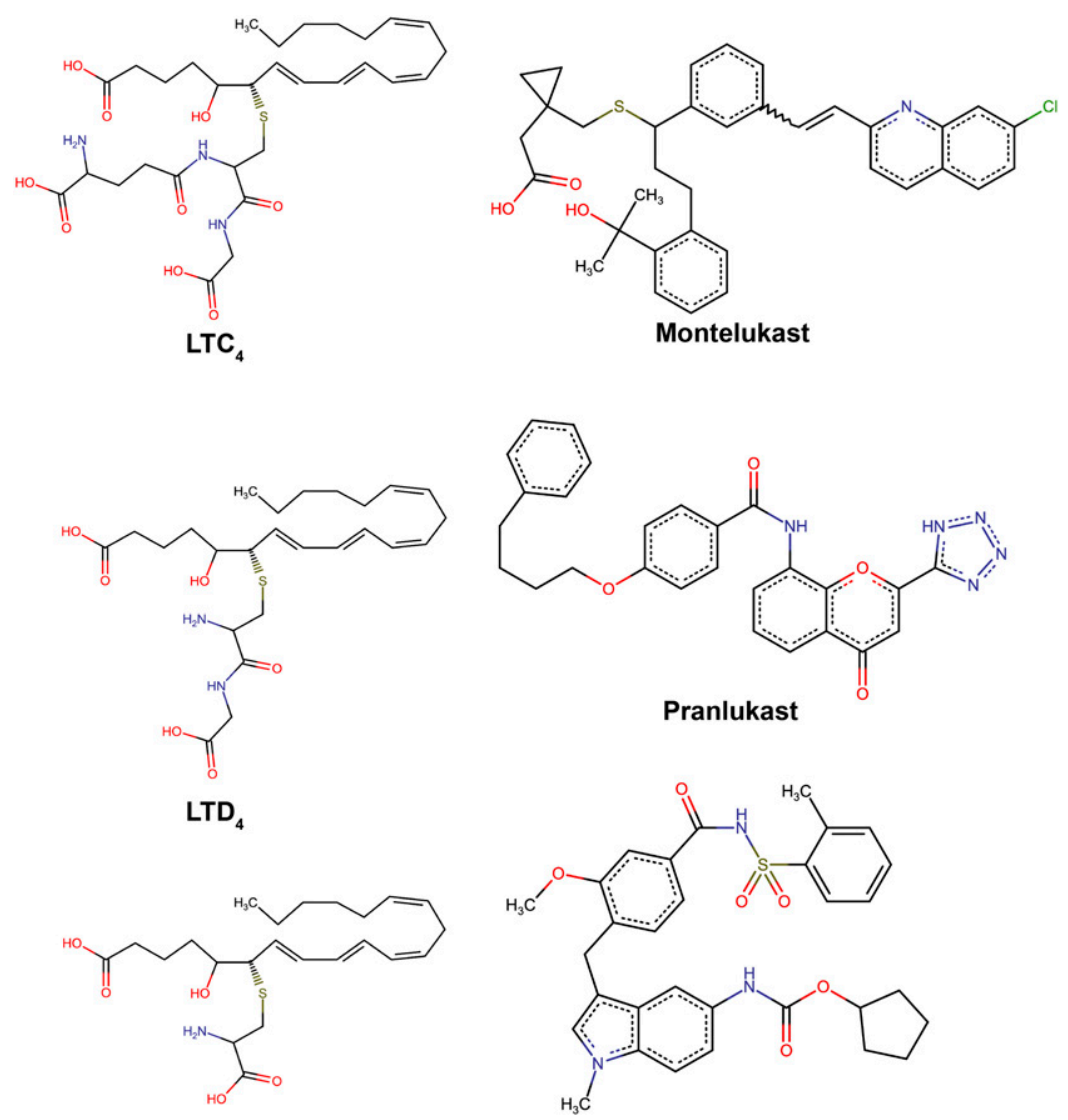

$\mathrm{LTE}_{4}$

Zafirlukast

Fig. 5. Chemical structures of CysLTs and LTRAs.

as the main targets for $\mathrm{LTC}_{4}$ and $\mathrm{LTD}_{4}$ (Labat et al., 1992; Lynch et al., 1999; Takasaki et al., 2000).

CysLTR $_{1}$ and CysLTR 2 have different tissue expression profiles and different preferences for CysLTs (Laidlaw and Boyce, 2012; Liu and Yokomizo, 2015; Yokomizo et al., 2018). CysLTR 2 has been confirmed to be highly expressed in the heart and coronary smooth muscle cells, where little CysLTR 1 was detected. Both receptors are expressed in innate and adaptive immune cells and lung smooth muscle cells, although they play different roles in asthma and other inflammatory conditions. CysLTR 1 prefers $\mathrm{LTC}_{4}$ over $\mathrm{LTD}_{4}$, whereas $\mathrm{LTC}_{4}$ and $\mathrm{LTD}_{4}$ are equally potent agonists of CysLTR 2 . Both receptors exhibit low affinities for $\mathrm{LTE}_{4}$. Multiple studies suggested the existence of another receptor for $\mathrm{LTE}_{4}$, the molecular identity of which is still in debate (Laidlaw and Boyce, 2012). Both CysLTR 1 and CysLTR Cainly signal $_{2}$ through the $\mathrm{G}_{\mathrm{q} / 11}$ family to evoke cellular $\mathrm{Ca}^{2+}$ responses and cause bronchoconstriction (Gorenne et al., 1998; Snetkov et al., 2001). CysLTR 1 has also been shown to induce signaling events that are sensitive to pertussis toxin in some cells, including ASM cells, indicating the involvement of the $\mathrm{G}_{\mathrm{i} / \mathrm{o}}$ family (Pollock and Creba, 1990; Hoshino et al., 1998; Mellor et al., 2003; Jiang et al., 2006; Ravasi et al., 2006). These studies suggested that $\mathrm{G}_{\mathrm{i} / \mathrm{o}}$-mediated signaling leads to the activation of MAPK.
CysLTs act on the CysLTRs to exert their roles in asthma through multiple mechanisms. During the 1980 s, a number of laboratories reported the potent bronchoconstriction action of $\mathrm{LTC}_{4}$ and $\mathrm{LTD}_{4}$ on isolated human bronchi or in humans (Dahlén et al., 1980, 1983; Holroyde et al., 1981; Weiss et al., 1982a; Bisgaard and Groth, 1987). Several studies showed that CysLTs are hundreds or even thousands of times more potent than histamine in inducing bronchial contraction (Weiss et al., 1982a,b; Drazen, 1988). Compared with $\mathrm{LTC}_{4}$ and $\mathrm{LTD}_{4}, \mathrm{LTE}_{4}$ is a weaker bronchoconstrictor, as suggested by several studies, although asthmatic patients showed an increased sensitivity to $\mathrm{LTE}_{4}$ (Davidson et al., 1987; Arm et al., 1990). The inherent tone of human airway tissues is believed to be maintained by the balance between contractile and vasodilating mediators (Ellis and Undem, 1994; Watson et al., 1997). Overproduction of CysLTs can enhance the contractile tone by inducing smooth muscle hyperplasia in asthma (Hui and Funk, 2002). The involvement of CysLTR $_{1}$ in the bronchoconstrictor effects of CysLTs is well validated by clinical trials with LTRAs that specifically target CysLTR ${ }_{1}$ (Matsuse and Kohno, 2014). Recently, Yonetomi et al. (2015) and Sekioka et al. (2015, 2017) demonstrated that CysLTR ${ }_{2}$ also plays important roles in CysLT-induced bronchoconstriction, suggesting 
a potential therapeutic benefit of blocking both CysLTR $_{1}$ and $\mathrm{CysLTR}_{2}$ in reducing bronchoconstriction.

CysLTs also play important roles in the inflammatory process involved in the pathogenesis of asthma. Laitinen et al. (1993) reported the effects of $\mathrm{LTE}_{4}$ in the recruitment of eosinophils and neutrophils in the airway mucosa. Other studies also demonstrated the positive effects of CysLTs, in particular $\mathrm{LTD}_{4}$, on the migration of eosinophils into the airways and proposed that CysLTs contributed to the airway eosinophilia (Foster and Chan, 1991; Smith et al., 1993; Henderson et al., 1996). This is partly due to the abilities of CysLTs to induce the expression of adhesion molecules on leukocytes (Kanwar et al., 1995; Pedersen et al., 1997; Fregonese et al., 2002; Nagata et al., 2002). Lee et al. (2000) showed that CysLTs could stimulate eosinophil survival, which was reversed by a LTRA. Cumulatively, these studies suggested the chemotactic effects of CysLTs for eosinophils in asthma. CysLTs have also been shown to simulate the generation of potent inflammatory mediators by eosinophils such as eosinophilic cationic protein, eosinophilic protein $\mathrm{X}$, neurotoxin, and superoxide radicals (Peters-Golden et al., 2006). Treatment with $\mathrm{LTD}_{4}$ antagonists, which are CysLTR $_{1}$ selective, was shown to reduce airway eosinophil influx and eosinophilia in several species (Laitinen et al., 1993; Spada et al., 1994; Turner et al., 1994; Underwood et al., 1996). In vitro studies also showed that antagonists of CysLTRs could downregulate type 2 cytokines and thus reduce $\mathrm{T}_{\mathrm{H}} 2$-mediated inflammation (Tohda et al., 1999; Bandeira-Melo et al., 2002; Mellor et al., 2002). In addition, several type 2 cytokines, such as IL-4 and IL-5, could enhance the synthesis of CysLTs by modulating the location and activities of 5-LOX and $\mathrm{LTC}_{4}$ synthase (Cowburn et al., 1999; Ochensberger et al., 1999). IL-4, IL-5, and IL-13 have also been shown to upregulate the expression of CysLTR $_{1}$ in several leukocytes and smooth muscle cells, providing a positive feedback mechanism to augment the inflammatory responses induced by CysLTs (Thivierge et al., 2000; Mellor et al., 2002; Espinosa et al., 2003).

The potent bronchoconstriction and proinflammatory effects of CysLTs lead to their critical roles in the formation and exacerbation of mucosal edema caused by increased microvascular permeability and leakage and the increased secretion of mucus, which are the main underlying mechanisms of airway obstruction. Early studies demonstrated that $\mathrm{LTC}_{4}$ and $\mathrm{LTD}_{4}$ could induce intense vascular constriction and dosedependent extravasation of macromolecules in the hamster cheek pouch (Dahlén et al., 1981). Later, $\mathrm{LTE}_{4}$ was shown to increase the vascular permeability by inducing the contraction of endothelial cells in guinea pigs (Joris et al., 1987). LTD 4 has also been shown to mediate dose-dependent microvascular leakage and bronchoconstriction in guinea pigs (Bochnowicz and Underwood, 1995). Studies using gene-modified mice confirmed the involvement of $\mathrm{CysLTR}_{1}$ and $\mathrm{CysLTR}_{2}$ in the CysLT-enhanced vascular permeability (Maekawa et al., 2002; Beller et al., 2004; Moos et al., 2008). LTRAs could reverse such effects of CysLTs in several studies, further demonstrating the involvement of CysLTRs (Nakagawa et al., 1992; Bochnowicz and Underwood, 1995). The effects of $\mathrm{LTC}_{4}$ and $\mathrm{LTD}_{4}$ in inducing mucus production in the airways in vitro and in vivo have also been well documented (Marom et al., 1982; Coles et al., 1983; Johnson et al., 1983).

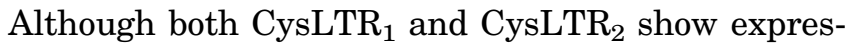
sion in lung smooth muscle cells and eosinophils, CysLTR $_{1}$ is considered to be the major target for the pathophysiological action of CysLTs in asthma. This was largely based on the results from clinical inves-

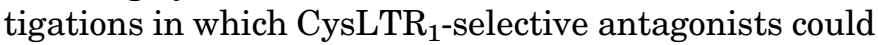
reverse most of the detrimental effects of CysLTs (Hui and Funk, 2002; Capra et al., 2007). In contrast, several lines of evidence suggested that the activity of CysLTR

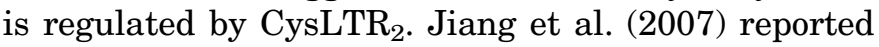
that CysLTR $_{2}$ forms heterodimer with CysLTR $_{1}$ and negatively regulates the mitogenic responses induced

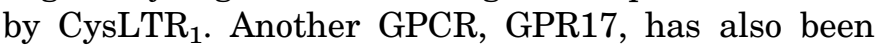
shown to dampen the function of $\mathrm{CysLTR}_{1}$ (Maekawa et al., 2009).

\section{B. Currently Used Leukotriene Receptor Antagonist Drugs and Their Pharmacological Properties}

The current LTRAs used in the treatment of asthma and other inflammatory diseases specifically target CysLTR $_{1}$. To date, three LTRAs, montelukast, pranlukast, and zafirlukast, are used in the clinics (Fig. 5). Pranlukast was the first marketed LTRA worldwide, although it is mostly used in Japan (Barnes et al., 1997). Zafirlukast was the first LTRA approved by FDA and used in the United States in 1990s, followed by the later approval of montelukast. All three compounds show high affinities for CysLTR $_{1}$, although they exhibit different pharmacological actions in blocking the action of $\mathrm{LTC}_{4}$ and $\mathrm{LTD}_{4}$ (Ravasi et al., 2002).

The primary in vivo effects of LTRAs are the inhibition of bronchoconstriction and inflammation. Fukushima et al. (1998) showed that LTRAs were more potent in reducing antigen-induced contraction of human lung parenchyma compared with other therapies, including PG synthase and receptor inhibitors and antihistamine reagents in in vitro assays. The antiinflammatory effects of LTRAs have been well documented. At therapeutic concentrations, LTRAs reduce the generation of reactive oxygen species and the release of a number of inflammatory mediators (Diamant and Sampson, 1999). In fact, a large number of studies that examined the inflammatory effects of CysLTs and CysLTRs used LTRAs as competitors to reverse the inflammatory action of CysLTs.

Some studies suggested the involvement of CysLTR ${ }^{-}$ independent mechanisms for the anti-inflammatory 
action of LTRAs (Tintinger et al., 2010; Theron et al., 2014), which include the inhibitory effects of LTRAs on cyclic nucleotide PDEs, transcription factor nuclear factor-?B, $\mathrm{PGE}_{4}$ synthase, and 5-LOX. However, the clinical significance of those mechanisms needs to be further elucidated.

1. Montelukast. Montelukast is the most widely prescribed LTRA in the United States and Europe, and the clinical significance of LTRAs in asthma was largely established based on the studies with montelukast. In vitro studies indicate that montelukast is associated with low nanomolar affinities, and it can specifically block the binding of $\mathrm{LTC}_{4}$ and $\mathrm{LTD}_{4}$ to cells expressing CysLTR $_{1}$ (Jones et al., 1995). The initial large-scale clinical evaluation of montelukast during the 1990s established its efficacy in asthma and led to its approval (Schoors et al., 1995; Cheng et al., 1996; De Lepeleire et al., 1997; Reiss et al., 1997). Several comparative studies suggested that montelukast could improve asthma control, reduce the rate of asthma exacerbation, and protect against bronchoconstriction (Leff et al., 1998; Reiss et al., 1998; Malmstrom et al., 1999). One study also showed that montelukast provided significant clinical benefits in protecting against EIA (Leff et al., 1998).

Several studies have also compared montelukast to ICS because ICS is the first-line anti-inflammatory agent for asthma. Some of them showed a superior efficacy of low-dose ICS compared with montelukast, which may be associated with the involvement of patients with largely moderate-to-severe asthma (Laviolette et al., 1999; Busse et al., 2001). Later, in a small-scale study with patients with mild asthma, montelukast showed satisfying efficacy as a monotherapy with a clinically significant improvement of asthma control (McIvor et al., 2009). Noticeably, in this study, montelukast was associated with a much higher compliance to asthma therapy compared with ICS. This may be particularly important for treating asthma in the elderly.

Montelukast is most often used as an add-on therapy instead of monotherapy (Matsuse and Kohno, 2014). Early studies demonstrate that montelukast could provide additional benefits of improving symptoms and lung function when combined with ICS (Laviolette et al., 1999; Stempel, 2000; Vaquerizo et al., 2003). However, later studies that compared montelukast with LABAs in combined use with ICS generated mixed results (Fish et al., 2001; Vaquerizo et al., 2003; Deykin et al., 2007). It has been suggested that ICS with LABAs may provide higher efficacy compared with ICS with montelukast for short-term use, whereas these two methods resulted in similar outcomes for long-term use (Joos et al., 2008). Montelukast is associated with a higher safety profile and a lower rate of side effects compared with LABAs in long-term use (Joos et al., 2008). The higher safety profile of montelukast may be especially beneficial for treating asthma in children and adolescents (Bisgaard et al., 2009). In fact, all three LTRAs can be used in pediatrics with controlled dosages.

Montelulast as a representative LTRA has been studied in a number of asthma phenotypes, in which the level of inflammatory CysLTs is elevated due to specific factors or conditions such as smoking and exercise. Asthma patients who smoke may develop resistance to ICS (Thomson et al., 2004), and there is one study conducted in the 1990s demonstrating that habitual smoking is associated with increased levels of CysLTs (Fauler and Frölich, 1997). In a small-scale clinical study on mild asthma patients with selfreported smoking, the response to ICS was attenuated and montelukast as a monotherapy resulted in a greater improvement in lung function (Lazarus et al., 2007). A later clinical study with over 300 patients showed that although patients with a history of smoking less than 11 years tended to have more benefits with ICS, montelukast seemed to offer more benefits for patients with a long history of smoking (>11 years) (Price et al., 2013). Second, EIA or exercise-induced bronchoconstriction is frequent in children and especially in athletes. One study showed that montelukast could attenuate exercise-induced bronchoconstriction in children with asthma (Kemp et al., 1998). Although montelukast showed a lower efficacy compared with LABAs in treating EIA in one study (Raissy et al., 2008), it is associated with less side effects and does not lead to tolerance. For LABAs, if taken daily, tolerance may develop to the protective effects in EIA (Anderson, 2004). Third, montelukast has been studied in the treatment of aspirininduced asthma (AIA). Aspirin and other nonsteroidal anti-inflammatory drugs can inhibit the activity of cyclooxygenase and thus facilitate the metabolism of AA through 5-LOX pathways to generate excessive CysLTs. It was estimated that about $10 \%$ asthma patients on nonsteroidal anti-inflammatory drugs may develop AIA (Matsuse and Kohno, 2014). One study showed that aspirin-sensitive or aspirin-intolerant asthma patients had a greater level of $\mathrm{LTE}_{4}$ compared with the control group of asthma patients (Christie et al., 1991). Another small-scale clinical trial showed promising results of montelukast in treating AIA, in which montelukast as an add-on drug could further improve pulmonary function and asthma-specific quality of life over what could be achieved by ICS (Dahlén et al., 2002). Fourth, obesity is another factor that can result in systemic inflammation associated with adipose tissues, which includes the generation of CysLTs. One clinical study examined the relationship of asthma and body mass index and showed that in general the response of asthma patients to ICS decreased with increased body mass index, but the response to montelukast remained stable (Giouleka et al., 2011). This may be an example of where asthma endotype plays 
a role in that obesity-related asthma does not have a typical type 2 cytokine profile, which is most responsive to ICS therapy.

2. Pranlukast. In vitro studies showed that pranlukast is a potent and selective antagonist for CysLTR that could dose-dependently reverse the effects of $\mathrm{LTC}_{4}$ and $\mathrm{LTD}_{4}$ on isolated human bronchial smooth muscle (Nakagawa et al., 1992; Yamaguchi et al., 1992). In one study, pranlukast showed lower potency in competing with the binding of ${ }^{3} \mathrm{H}-\mathrm{LTC}_{4}$ to $\mathrm{CysLTR}_{1}$ compared with montelukast (Ravasi et al., 2002). Consistently, for the clinical use, the dosage of pranlukast $(\sim 200 \mathrm{mg}$ once or twice daily) is much higher than the dosage of montelukast ( $\sim 10-20 \mathrm{mg}$ once daily) for treating asthma in adults.

Animal studies with pranlukast confirmed its potent anti-inflammatory and antibronchospasm effects as a result of specifically blocking CysLT binding (Obata et al., 1987, 1992; Nakagawa et al., 1992, 1993; Kurosawa et al., 1994; Bochnowicz and Underwood, 1995). The anti-inflammatory effects of pranlukast on eosinophils were examined and confirmed in a few clinical trials (Horiguchi et al., 1999; Ishioka et al., 1999; Yamauchi et al., 2001). In several studies, pranlukast showed significant efficacy in reversing bronchoconstriction caused by various stimuli, including $\mathrm{LTD}_{4}$, dust mite, aspirin, exercise, and even alcohol (Abraham et al., 1993; Hamilton et al., 1998; Ishioka et al., 2000; Suguro et al., 2000; Yoshida et al., 2000b; Myou et al., 2001; Obase et al., 2001, 2002). Some other studies also showed the effectiveness of pranlukast in reducing bronchial hypersensitiveness, another important feature of asthma, caused by various factors (Nakamura et al., 1998; Ishioka et al., 2000; Yoshida et al., 2000a; Yamauchi et al., 2001; Sagara et al., 2009). Because the current first-line method for treating asthma is ICS or ICS plus LABAs, a number of recent studies tested whether pranlukast could provide additional clinical benefits when used with ICS or ICS plus LABAs. These studies demonstrated that pranlukast could further reduce eosinophilic airway inflammation and thus might further improve lung function (Ohbayashi et al., 2009; Yasui et al., 2012). Indeed, a long-term follow-up study suggested that pranlukast provided benefits of improving lung function that lasted for more than 1 year if taken daily (Yanagawa et al., 2004).

Similar to montelukast, pranlukast has been studied in a number of clinical trials to examine its effects in different asthma phenotypes. The efficacy of pranlukast compared with placebo or antihistamine was confirmed in a few clinical trials with mild to moderate adult asthma patients (Barnes et al., 1997; Barnes and Pujet, 1997; Yoo et al., 2001). Several small-scale studies also showed that pranlukast was effective for patients with moderate to severe asthma treated with ICS or who are refractory to ICS (Yokoyama et al., 1998; Kohrogi et al., 1999; Tomari et al., 2001). The clinical efficacy of pranlukast in pediatric asthma was examined and proven in Japan (Keam et al., 2003). One study demonstrated that pranlukast monotherapy showed a clinical efficacy comparable to that of ICS monotherapy for elderly patients with mild asthma (Horiguchi et al., 2007).

3. Zafirlukast. Although described as a selective and competitive antagonist of $\mathrm{CysLTR}_{1}$ (Krell et al., 1990), zafirlukast is a relatively less studied LTRA compared with montelukast and pranlukast. Early studies that demonstrated its ability to reduce eosinophil-related inflammation and bronchoconstriction were conducted in animals (Krell et al., 1990, 1994). One in vitro study showed that zafirlukast is as potent as montelukast in blocking the binding of ${ }^{3} \mathrm{H}-\mathrm{LTD}_{4}$ to CysLTR $_{1}$ in isolated human lung parenchyma (Ravasi et al., 2002). The same study also demonstrated that, unlike montelukast and pranlukast, zafirlukast was unable to interfere with the binding ${ }^{3} \mathrm{H}-\mathrm{LTC}_{4}$ in the same experimental settings, suggesting a potentially different pharmacological action of zafirlukast compared with montelukast and pranlukast, such as a

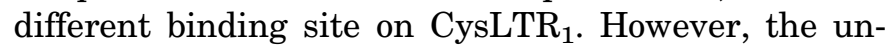
derlying mechanism is not clear (Dunn and Goa, 2001).

The efficacy of zafirlukast in asthma has been confirmed in a number of clinical trials (Dunn and Goa, 2001). Compared with placebo, zafirlukast provided sustained clinical benefits for patients with mild to moderate asthma in a large-scale clinical study (Fish et al., 1997). As a monotherapy, zafirlukast also showed significant benefits for patients with severe and persistent asthma (Kemp et al., 1999). The effects of zafirlukast as an add-on therapy were also evaluated. One study showed that zafirlukast could improve lung function and reduce the risk of exacerbation for patients who were already on high-dose ICS (Virchow et al., 2000). However, a few studies showed that zafirlulast was less effective as an add-on drug compared with salmeterol, a LABA, in combined use with ICS (Busse et al., 1999; Nelson et al., 2001). The clinical benefits, safety, and tolerability of zafirlukast for children with mild to moderate asthma were evaluated and proven in several studies (Pearlman et al., 2000; Dunn and Goa, 2001).

\section{Structural Insights into Drug Action}

Montelukast, pranlukast, and zafirlukast exhibit distinct chemical scaffolds with limited similarity (Fig. 5). This is in contrast to $\beta_{2}$-agonists, which share a relatively conservative chemical feature. The first marketed LTRA, pranlukast, was developed based on compounds from random screening (Nakai et al., 1988; Bernstein, 1998). The structure and activity relationship (SAR) studies for developing pranlukast were done in reference to the structure of endogenous CysLTs. Other research efforts for developing antagonists of CysLTRs were focused on direct modifications of 
Wendell et al.

CysLTs (Bernstein, 1998). The aims were to modify the unstable polyene chain of CysLTs and to switch the agonists into antagonists. Such efforts led to the discovery of a series of lead compounds, which further led to the development of montelukast and zafirlukast through extensive SAR studies. Although the overall chemical structures of LTRAs are distinct from CysLTs, in montelukast, there is a cyclopropyl-acetic acid group attached to the main chain through a sulfanyl group, mimicking the Cys-Gly peptide moiety of $\mathrm{LTD}_{4}$.

To date there are no high-resolution structures of CysLTRs that have been solved experimentally. CysLTRs belong to the $\delta$-subgroup of class A GPCRs, whereas other leukotriene receptors and PG receptors belong to the $\alpha$ - and $\gamma$-subgroups (Fredriksson et al., 2003). This may suggest a novel mechanism for CysLTRs to recognize endogenous lipid ligands compared with other eicosanoid GPCRs. The closest phylogenetic neighbors of CysLTRs with solved structures are proteaseactivated receptors 1 and 2 (PAR1 and PAR2) (Zhang et al., 2012; Cheng et al., 2017). Two purinergic receptors, $\mathrm{P}_{2} \mathrm{Y}_{12}$ and $\mathrm{P}_{2} \mathrm{Y}_{1}$, with solved structures are also closely related to CysLTRs (Zhang et al., 2014a,b, 2015). However, those structures revealed very diverse binding modes of antagonists. It seems that antagonists of PARs and purinergic receptors could recognize multiple ligand-binding sites inside or on the surface of the 7TM bundle. Therefore, it is difficult to gain insight into the binding of LTRAs to CysLTR $_{1}$ based on the published structures of GPCRs in the $\delta$-subgroup. Because currently used LTRAs are with diverse chemical structures with no obvious conserved features, it is likely that they engage in different modes of interactions with the receptor or even occupy different ligand-binding sites. Considering the partial chemical similarity of montelukast with $\mathrm{LTD}_{4}$, montelukast may occupy the same orthosteric site as $\mathrm{LTD}_{4}$.

Although there is no structure of CysLTR bound to $_{1}$ montelukast, there is one structure of montelukast bound to the xenobiotic metabolizing enzyme, cytochrome P450 2C8 (Schoch et al., 2008) (Supplemental Material 1-PDB ID 2NNI). In this structure, montelukast showed a tripartite binding mode. The three branches of montelukast occupy three different binding pockets. The authors suggested that the high complementarities of montelukast to the ligand-binding cavity in shape, size, hydrophobicity, and polarity lead to the high affinity of montelukast for this enzyme. It will be interesting to see whether montelukast adopts

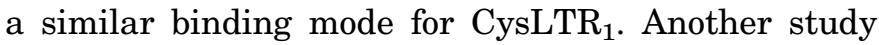
reported structural modeling of $\mathrm{CysLTR}_{1}$ bound to LTRAs (Bandaru et al., 2014). The authors further used this structure to do virtual screening to discover new CysLTR $_{1}$ antagonists. The results predicted a ligandbinding mode in which the ligand formed extensive interactions with the extracellular regions of TM4, TM5, TM7, as well as ECL2.

\section{Potential G Protein-Coupled Receptor-Targeting Drugs for Asthma in Late- Stage Clinical Trials}

\section{A. New G Protein-Coupled Receptor Targets for Developing Asthma Drugs}

Although current therapeutic methods including the combined use of ICS with LABAs are highly effective in most asthma patients, there is still a need for developing new therapies (Adcock et al., 2008; Barnes, 2010b; Brigden et al., 2016; Fajt and Wenzel, 2017; Gross and Barnes, 2017; Zhu et al., 2018). There are a number of proinflammatory mediators, including PGs, leukotrienes, and chemokines, which play important roles in the pathogenesis of asthma and bind GPCRs. Therefore, there have been continuous research efforts in the past several decades in developing drugs targeting these GPCRs to better control inflammation for treating asthma. Although some of those efforts were successful, such as the development of LTRAs, many of them were not.

Besides CysLTs, another leukotriene, $\mathrm{LTB}_{4}$, which does not contain a glutathione moiety, has been implicated in the inflammatory process of asthma (Gelfand, 2017). There are two GPCRs for $\mathrm{LTB}_{4}$, leukotriene receptor $\left(\mathrm{BLT}_{1}\right)$ as the high-affinity receptor and $\mathrm{BLT}_{2}$ as the low-affinity receptor. $\mathrm{BLT}_{1}$ is highly expressed in $\mathrm{CD}^{+} \mathrm{T}$ cells and DCs and promotes chemotaxis and generation of Th2 cytokines (Yamaoka and Kolb, 1993; Arcoleo et al., 1995). The effects of $\mathrm{LTB}_{4}-\mathrm{BLT}_{1}$ signaling axis in AHR have been proven in a number of animal models (Vargaftig and Singer, 2003; Miyahara et al., 2005; Terawaki et al., 2005). Although some studies showed efficacy of $\mathrm{BLT}_{1}$ antagonists in rodent and nonhuman primate models of asthma (Turner et al., 1996; Hicks et al., 2010; Waseda et al., 2011), clinical trials with $\mathrm{BLT}_{1}$ antagonists all failed or were not being pursued further (Hicks et al., 2007).

Over 50 different chemokines can be divided into four groups: CXC, CC, C, and CX3C (Rossi and Zlotnik, 2000; Castan et al., 2017). They signal through more than 20 GPCRs as chemokine receptors to result in the chemotaxis and recruitment of immune cells to initiate various inflammatory processes (Castan et al., 2017). In addition, chemokine receptors also play important roles in lung biology such as repair and remodeling (Tomankova et al., 2015). Despite intensive research efforts, very few drugs targeting chemokine receptors have made it to the market, and none of them are currently used for asthma (Castan et al., 2017). Among all chemokine receptors, CCR3 is the primary chemokine receptor expressed on eosinophils and $\mathrm{T}_{\mathrm{H}} 2$ cells and mediates the migration and activation of those immune cells (Pease, 2011; Castan et al., 2017). Therefore, it has long been suggested to be a potential drug target for asthma. The development of small-molecule antagonists of CCR3 as drugs has been proven difficult. 
Instead, an antisense nucleotide drug ASM8, which can downregulate CCR3, has been developed and studied in a few clinical trials (Pease and Horuk, 2014). Several studies showed that ASM8 could attenuate allergen-induced eosinophilic inflammation in asthmatic patients (Gauvreau et al., 2008, 2011; Imaoka et al., 2011). However, to the best of our knowledge, further clinical development of ASM8 for treating asthma was not reported. Another chemokine receptor, CXCR2, has been shown to regulate the production of type 2 cytokines during inflammation (Matsuda et al., 2008). One study suggested a critical role of CXCR2 in pathogen-induced persistent asthma in mice (Schuh et al., 2002). A number of CXCR2 antagonists have been studied clinically. Although one study with compound SCH527123 showed that CXCR2 antagonism could reduce neutrophils in patients with severe asthma (Nair et al., 2012), another phase II study with compound AZD5069 showed no effects in reducing the frequency of exacerbations in patients with uncontrolled severe asthma, raising the question about the roles of CXCR2 in severe asthma (O'Byrne et al., 2016). To the best of our knowledge, currently there are no active late-stage clinical trials with chemokine receptor antagonists for asthma registered on clinicaltrials.gov.

In humans, there are nine GPCRs characterized to date for the endogenous PGs $\mathrm{D}_{2}, \mathrm{E} 2, \mathrm{~F}_{2}, \mathrm{I}_{2}$, and thromboxane $\mathrm{A}_{2}\left(\mathrm{PGD}_{2}, \mathrm{PGE}_{2}, \mathrm{PGF}_{2}, \mathrm{PGI}_{2}\right.$, and $\left.\mathrm{TXA}_{2}\right)$ (Woodward et al., 2011). Although a number of $\mathrm{PG}$ receptors have been shown in vitro and in animal models to play important roles in inflammation (Peebles, 2019), to date only compounds that target one PG receptor, $\mathrm{PGD}_{2}$ receptor 2 (DP2, also named CRTH2), have advanced to phase III clinical trials for treating asthma.

The roles of GPCRs for PG, leukotriene, and chemokines in asthma have been reviewed in details by numerous papers elsewhere. In this work, we hope to provide a review of drug candidates targeting DP2 as a potential new class of asthma drugs in late-stage clinical trials. We will also review a few compounds in late-stage trials that act on existing GPCR targets for asthma.

\section{B. Prostaglandin $D_{2}$ Receptor 2 and Its Antagonists}

1. The Roles of Prostaglandin $D_{2}$ Receptor 2 in Asthma. AA can be metabolized by COX and PG synthases to generate various PG molecules (Peebles, 2019). $\mathrm{PGD}_{2}$ is synthesized by the PGD synthase from its precursor $\mathrm{PGH}_{2}$. In turn, $\mathrm{PGD}_{2}$ is further metabolized to other PGs, through enzymatic processes, which include 15deoxy- $\Delta^{12,14}-\mathrm{PGD}_{2}, \quad 13,14$-dihydro-15-keto-PGD $2,11 \beta$ $\mathrm{PGF}_{2 \alpha}$, and $\Delta^{12}-\mathrm{PGD}_{2}$, and nonenzymatically to $\mathrm{PGJ}_{2}$ and corresponding metabolites. During inflammatory processes, $\mathrm{PGD}_{2}$ is primarily produced by mast cells as well as other immune cells, including eosinophils, macrophages, DCs, and $\mathrm{T}_{\mathrm{H}} 2$ cells (Domingo et al., 2018). Two GPCRs mediate the cellular functions of $\mathrm{PGD}_{2}$ : $\mathrm{PGD}_{2}$ receptor 1 and 2 (DP1 and DP2). DP2 is also commonly named as CRTH2, which is short for 3chemoattractant receptor-homologous molecule expressed on $\mathrm{T}_{\mathrm{H}} 2$ cells (Nagata and Hirai, 2003). High concentrations of $\mathrm{PGD}_{2}$ can also act on the thromboxane receptor (TP) (Coleman and Sheldrick, 1989). Interestingly, DP1 and DP2 are phylogenetically distant, even though they recognize the same endogenous ligand. DP1 is closely related to other PG receptors, whereas DP2 is more akin to a group of chemoattractant GPCRs such as the receptors for anaphylatoxins C5a and C3a, formyl peptide receptors (FPRs), and BLTs (Fredriksson et al., 2003). Also, DP1 mainly couples to $G_{\mathrm{s}}$ family to stimulate cAMP production, whereas DP2 couples to $G_{i}$ family to reduce cAMP levels and induce $\mathrm{Ca}^{2+}$ responses.

The signaling of DP1 and DP2 in response to $\mathrm{PGD}_{2}$ plays different roles in inflammation (Hata and Breyer, 2004; Tanaka et al., 2004; Kostenis and Ulven, 2006; Pettipher, 2008). DP1 is mainly expressed in vascular smooth muscle cells, platelets, and DCs. DP1 signaling contributes to the pathologic changes of blood flow in inflammation and regulates the polarization of $\mathrm{T}_{\mathrm{H}} 2$ cells (Pettipher, 2008). The role of DP1 in inflammation is not completely understood and is controversial. A few studies suggested that the signaling of DP1 could inhibit the chemotaxis and activation of eosinophils, basophils, and DCs, therefore eliciting antiinflammatory actions (Angeli et al., 2004). Several in vivo studies showed that DP1 activation could be either proinflammatory or anti-inflammatory depending on different experimental methods, pathologic conditions, or pharmacological tools used (Matsuoka et al., 2000; Hammad et al., 2003; Angeli et al., 2004; Arai et al., 2004; Oguma et al., 2004; Kostenis and Ulven, 2006). In contrast, DP2 signaling has been consistently shown to be proinflammatory. DP2 is highly expressed in $\mathrm{T}_{\mathrm{H}} 2$ cells, including ILC2 cells, eosinophils, and basophils, and regulates the chemotaxis of those cells (Nagata et al., 1999; Hirai et al., 2001; Chang et al., 2014; Xue et al., 2014). In the past two decades, a plethora of studies have confirmed the critical role of DP2 signaling in inflammation.

$\mathrm{PGD}_{2}$-induced DP2 signaling has been shown to upregulate the production of type 2 cytokines, including IL-4, IL-5, and IL-13 by $\mathrm{T}_{\mathrm{H}} 2$ cells and ILC2 cells through a $\mathrm{PGD}_{2}$ dose-dependent manner in in vitro studies (Xue et al., 2005, 2014). It can also promote the chemotaxis and migration of $\mathrm{T}_{\mathrm{H}} 2$ cells, ILC2 cells, and eosinophils (Heinemann et al., 2003; Gyles et al., 2006; Xue et al., 2012, 2014). Xue et al. (2009) showed that $\mathrm{PGD}_{2}$ could promote prolonged inflammation by inhibiting apoptosis of $\mathrm{T}_{\mathrm{H}} 2$ cells through DP2. The activation of eosinophils by DP2 has also been demonstrated in a number of studies. Those studies showed that $\mathrm{PGD}_{2}$ signaling through DP2 on the surface of eosinophils could lead to shape change and promote degranulation and cytokine production (Gervais et al., 2001; 
Sykes et al., 2016; Sandham et al., 2017). In addition, type 2 cytokines induced by DP2 signaling can have positive effects on the activation and migration of eosinophils, further contributing to excessive inflammation. All of those studies indicated a strong linkage of DP2 to inflammation, especially eosinophil-associated inflammation.

Additional studies have demonstrated a critical role of DP2 in asthma. Early studies demonstrated that allergen challenge could lead to the release of $\mathrm{PGD}_{2}$ into human airways, especially in asthma patients (Murray et al., 1986; Wenzel et al., 1991). Fajt et al. (2013) showed that $\mathrm{PGD}_{2}$ and DP2 pathway is upregulated in patients with severe and $\mathrm{T}_{\mathrm{H}} 2$-high asthma. $\mathrm{PGD}_{2}$ and DP2 pathway has also been shown to mediate respiratory virus-induced enhancement of inflammation and AHR (Shiraishi et al., 2008). The development of potent and specific DP2 antagonists has allowed studies of DP2 antagonism in tissues, animals, and patients, which provided direct evidence for the potential use of DP2targeting therapies for treating asthma (Kupczyk and Kuna, 2017).

2. Prostaglandin $\mathrm{D}_{2}$ Receptor 2 Antagonists. The first nonlipid DP2 antagonist to be discovered was ramatroban (also named BAY-u3405), which was initially developed as a drug antagonizing TP (Sugimoto et al., 2003; Ishizuka et al., 2004) (Fig. 6). Although ramatroban is a nonselective DP2 antagonist, it is selective for DP2 over DP1. Studies using ramatroban confirmed that $\mathrm{PGD}_{2}$-induced eosinophilic inflammation in the airways was mediated by DP2, but not DP1 (Shiraishi et al., 2005). Later, a number of potent and selective DP2 antagonists were soon developed based on SAR studies of ramatroban involving research groups in academia and industry (Ly and Bacon, 2005). Those include a potent DP2 antagonist, CAY10471 (also named TM30089), which showed insurmountable antagonism on DP2 in in vitro studies (Mathiesen et al., 2006). It was then used in studies based on a mouse asthma model, which for the first time demonstrated the in vivo efficacy of DP2 antagonists in reducing eosinophilic inflammation in the airways (Uller et al., 2007). CAY10471 was also shown to inhibit chemotaxis of eosinophils from guinea pig bone marrow tissues (Royer et al., 2007).

During the 2000s, a number of DP2 antagonists were pushed into clinical studies for the treatment of asthma. A small-scale clinical study using compound OC000459 as a potent DP2 antagonist provided the first clinical evidence for the effectiveness of antagonizing DP2 in reducing airway inflammation and improving symptoms in patients with moderate to severe asthma (Barnes et al., 2012). Later, a larger clinical study using a lower dose of OC000459 again proved its efficacy in improving symptoms and lung function in asthma (Pettipher et al., 2014). Another study showed that OC000459 could effectively inhibit allergic inflammation in asthma patients (Singh et al., 2013). In all of those clinical studies, asthma patients without ICS treatment were included. Further clinical development of OC000459 is ongoing (Kupczyk and Kuna, 2017).

Other DP2 antagonists that have gone through clinical studies include BI 671800 (Fowler et al., 2017), AZD 1981 (Schmidt et al., 2013), AMG 853 (Liu et al., 2011), setipiprant (Fretz et al., 2013), and fevipiprant (Sykes et al., 2016) (Fig. 6). The results from those clinical studies were mixed. BI 671800 showed an effect of slightly improved lung function in steroid-naive asthma patients, but failed to show additional effects as an add-on therapy to ICS (Hall et al., 2015; Miller et al., 2017). The authors suggested that it might be due to the insufficient inhibition of DP2 signaling by BI 671800 even at a high dose. Two phase II clinical trials with AZD 1981 showed improved lung function and symptoms in asthma patients withdrawn from ICS treatment or patients with uncontrolled asthma despite ICS treatment (Kuna et al., 2016). However, another study indicated that AZD 1981 failed to show any clinical benefit as an add-on therapy to ICS plus LABA in patients with allergic asthma (Bateman et al., 2018). AMG 853 is a unique DP2 antagonist because it also antagonizes DP1. Even with a dual action on DP2 and DP1, AMG 853 did not show any benefit in improving lung function or symptoms as an add-on therapy to ICS in patients with poorly controlled moderate to severe asthma (Busse et al., 2013). For setipiprant, one small-scale study showed that it could reduce allergen-induced late asthmatic response in patients with allergic asthma, consistent with the findings for other DP2 antagonists (Diamant et al., 2014). Fevipiprant (also named QAW039) is the most advanced DP2 antagonist in clinical trials (White et al., 2018). One phase II trial showed that fevipiprant could reduce eosinophilic inflammation in a group of patients with uncontrolled moderate to severe asthma despite ICS treatment (Gonem et al., 2016). This was also the first study to show a positive effect of a DP2 antagonist in improving airway remodeling. Consistently, fevipiprant has been demonstrated to be efficacious in improving the end point of forced expiratory volume in patients with uncontrolled asthma who were on low-dose ICS (Bateman et al., 2017). Another phase II study showed that fevipiprant could improve lung function and asthma control in a small group of patients with severe asthma that was not well controlled by ICS plus LABA (Erpenbeck et al., 2016). Currently, fevipiprant is in several phase III clinical trials (White et al., 2018). If successful, it will be the first small-molecule asthma drug with a new mechanism over a decade.

Fevipiprant has been suggested to be a slow-dissociating DP2 antagonist based on radioactive ligand-binding assays (Sykes et al., 2016). Although the half-life of dissociation of fevipiprant determined in those assays was only 14.4 minutes, it was still significantly slower 

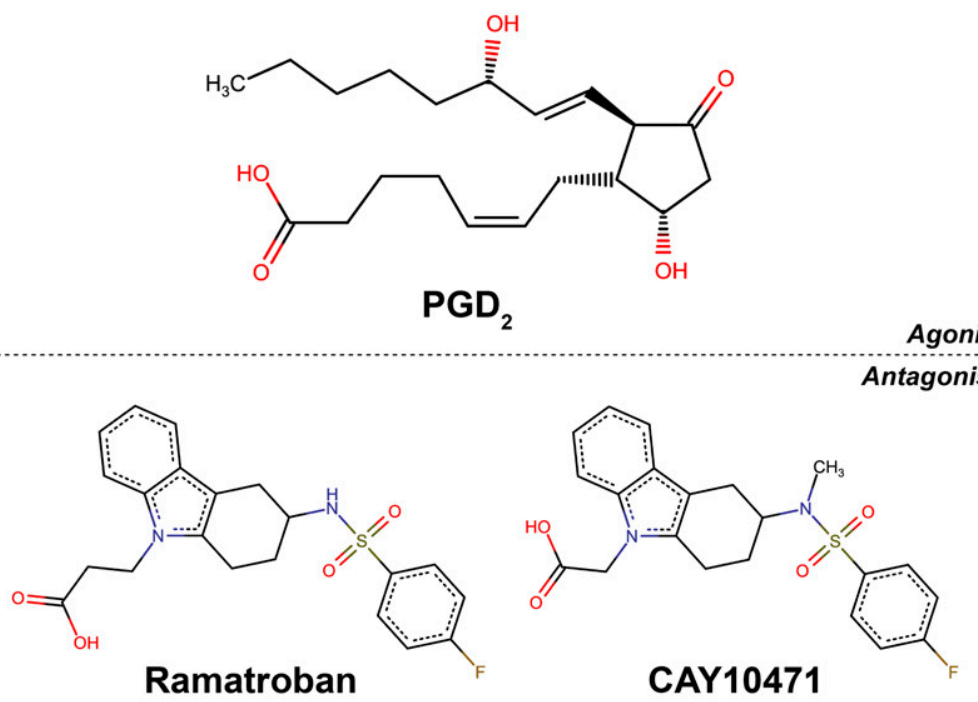

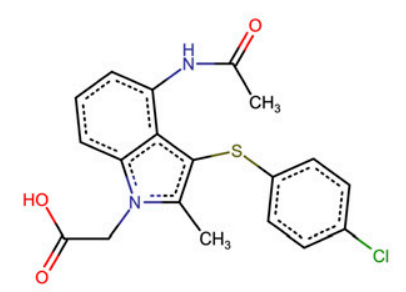

AZD1981

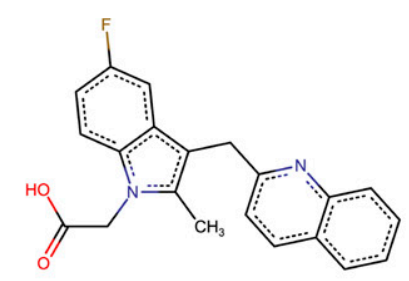

$0 C 000459$

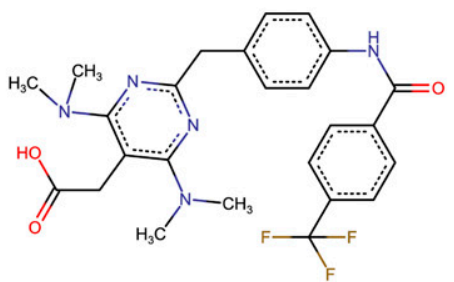

BI-671800<smiles>O=C(O)Cn1c2c(c3cc(F)ccc31)CN(C(=O)c1cccc3ccccc13)CC2</smiles>

Setipiprant<smiles>Cc1c(CC(=O)O)c2cccnc2n1Cc1ccc(S(C)(=O)=O)cc1C(F)(F)F</smiles>

Fevipiprant

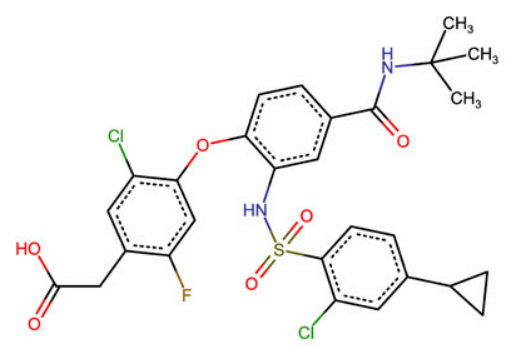

AMG853

Fig. 6. Chemical structures of $\mathrm{PGD}_{2}$ and $\mathrm{CRTH} 2$ antagonists.

than AZD 1981. OC000459 has been shown to be a highly reversible antagonist in the presence of $\mathrm{PGD}_{2}$ (Pettipher et al., 2012). Although highly speculative, it is possible that the receptor residence time is an important factor affecting the clinical efficacy of DP2 antagonists in asthma. During inflammation, locally generated $\mathrm{PGD}_{2}$ by mast cells and other immune cells could be at a high concentration, which may overcome the antagonistic action of highly reversible DP2 antagonists even though their concentrations in the plasma stay high. In this regard, for DP2 antagonists, a slow dissociation rate and long-lasting action may translate into a superior in vivo efficacy. Similar to such speculation, it has been suggested that for another GPCR, the C5a receptor, signaling of which promotes strong inflammatory responses, receptor residence time is more important than drug-likeness and bioavailability in determining the efficacy of its antagonists 
(Seow et al., 2016). For DP2, one compound named LAS191859 was reported to be an extremely slowdissociating antagonist with a half-life of dissociation more than 20 hours (Calbet et al., 2016). The authors showed that the long receptor residence time led to a long-lasting in vivo efficacy of LAS191859 even when the plasma level of the compound was low.

3. Structural Insights into the Action of Prostaglandin $D_{2}$ Receptor 2 Antagonists. Nearly all DP2 antagonists contain a carboxylate group like $\mathrm{PGD}_{2}$, which is believed to be a major pharmacophore. In many of them, the carboxylate group is attached to an aromatic group with one methyl group in between (Pettipher and Whittaker, 2012). Mathiesen et al. (2006) showed that the spacer between the carboxylate and the aromatic group of CAY10471 was important for ligand potency. Other than the carboxylate group, DP2 antagonists seem to exhibit a vast chemical diversity (Pettipher and Whittaker, 2012; Norman, 2014).

Recently, two crystal structures of human DP2 bound to two antagonists, CAY10471 and fevipiprant, were reported, which revealed the molecular details for the binding of both ligands (Wang et al., 2018) (Fig. 7) (Supplemental Material 1-PDB IDs 6D26 and 6D27). In the structures, the N-terminal region adopts a wellfolded structure stabilized by a disulfide bond with TM5. It results in a semi-occluded ligand-binding pocket and restricts the access to the ligand-binding pocket through the extracellular region. There is a gap between the extracellular regions of TM1 and TM7 as the only open end of the ligand-binding pocket, which was proposed to be the ligand entry port for endogenous ligands as well as synthetic antagonists. In the structures, the carboxylate group in both antagonists as the head group engages in a polar interaction network with several polar residues buried deeply in the ligandbinding pocket. Interestingly, a majority of residues in the ligand-binding pocket are actually aromatic residues, which form extensive hydrophobic and $\pi$-stacking interactions with the central aromatic group in CAY10471 or fevipiprant.

Although the overall binding poses of CAY10471 and fevipiprant are similar, their tail groups adopt different conformations and interact with different parts of the receptor. As a result, the tryptophan residue W283 of DP2 shows different conformations (Wang et al., 2018). In addition, docking of ramatroban, which shares a high chemical similarity with CAY10471, showed that the carboxylate group in ramatroban forms polar interactions with a different set of residues, resulting in a different binding pose of ramatroban compared with CAY10471. This explains the lower potency of ramatroban compared with CAY10471 (Mathiesen et al., 2006). Those observations imply a certain flexibility of the ligand-binding pocket, which should be an important consideration for structure-based drug design studies. Another interesting feature revealed by the
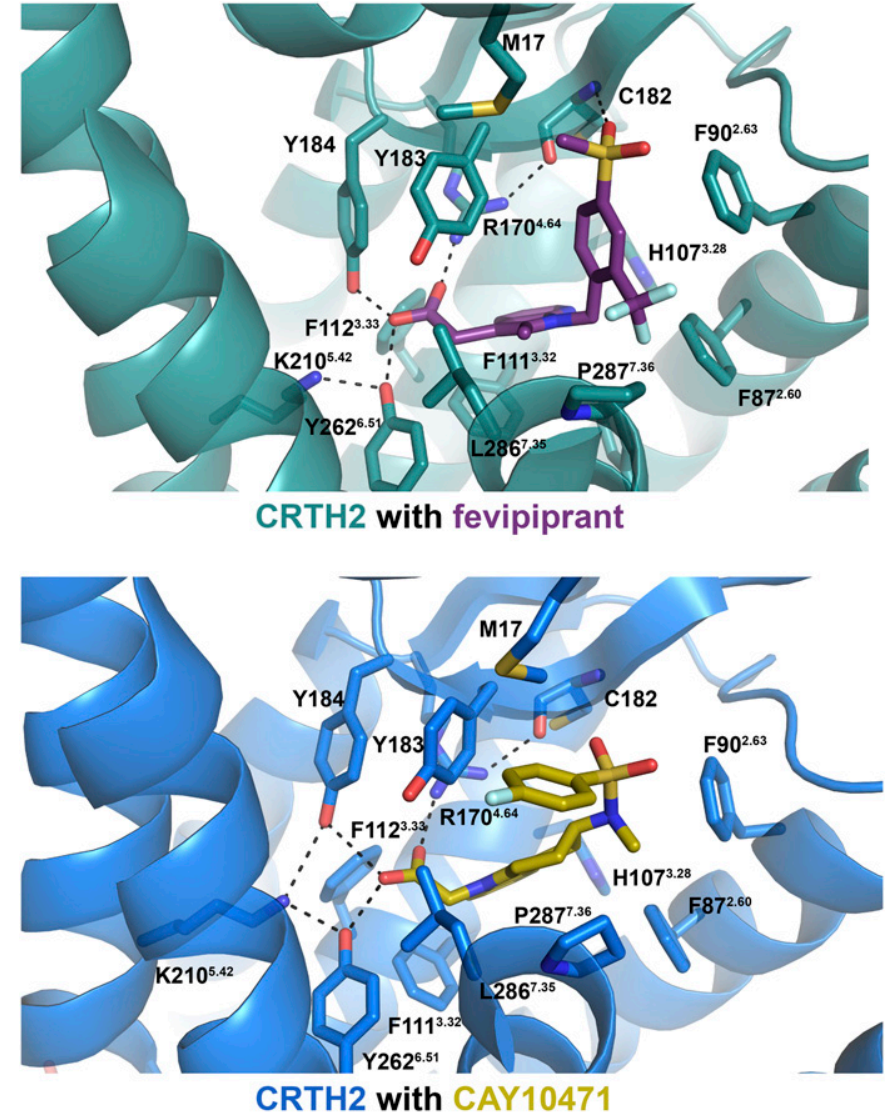

Fig. 7. Orthosteric ligand-binding site in CRTH2. The crystal structures of human CRTH2 bound to fevipiprant (left, Supplemental Material 1PDB ID 6D26) and CAY10471 (right, Supplemental Material 1-PDB ID 6D27) revealed the molecular details of ligand binding in the orthosteric ligand-binding site.

structures is that there are small molecules bound at the ligand entry port. This feature allowed the authors to propose a potential mechanism for the binding process of $\mathrm{PGD}_{2}$ (Wang et al., 2018). The authors also suggested that the ligand entry port in DP2 might provide an additional space for designing new DP2 antagonists. The molecular mechanism underlying receptor residence time for DP2 antagonists is not clear based on the crystal structures. A structure of DP2 with LAS191859 and MD simulation studies may provide additional insights and help with designing new longlasting DP2 antagonists.

\section{Potential Asthma Drugs Acting on Existing Asthma Targets}

Fixed-dose combinations of inhaled corticosteroids and LABAs have been commonly recommended for moderate to severe asthmatic patients. To improve the patients' adherence and the control of disease, there has been considerable interest for the next generation of inhaled $\beta_{2}$-agonists, maintaining over 24 hours of bronchodilation thus limited to daily use (ultra-LABAs). Although a few ultra-LABAs have already hit the market, at present there are still several ultra-LABAs 
in different stages of clinical development, such as abediterol and bedoradrine (Fig. 8).

1. Abediterol. The structure of abediterol contains both the lipophilic tail moiety similar to that of salmeterol and vilanterol and the quinoline-2-one moiety similar to that of indacaterol and olodaterol (Aparici et al., 2012). Abediterol is a full agonist at the human $\beta_{2} \mathrm{AR}$ [Emax (maximum effect) $=91 \% \pm 5 \%$ of the maximal effect of isoproterenol]. The potency and onset of action of abediterol in isolated human bronchi $\left(\mathrm{EC}_{50}\right.$ $=1.9 \pm 0.4 \mathrm{nM}$; onset $\mathrm{t}_{1 / 2}=7-10$ minutes) are similar to those of formoterol, whereas its duration of action (halflife $=690$ minutes) is more prolonged. In dogs, abediterol showed a greater safety margin than salmeterol and formoterol (5.6 vs. 3.3 and 2.2). Selectivity for $\beta_{2}$ over other $\beta$-adrenergic receptor subtypes determined by calculating $\beta_{1} / \beta_{2}$ and $\beta_{3} / \beta_{2} \quad \mathrm{IC}_{50}$ and $\mathrm{EC}_{50}$ ratios indicated that both salmeterol and abediterol have high selectivity profiles over the human $\beta_{1} \mathrm{AR}$ and $\beta_{3} \mathrm{AR}$, better than formoterol. In general, the preclinical data of abediterol suggested that it is a potent and selective $\beta_{2}$-agonist with rapid onset and long duration of action, together with a favorable cardiovascular safety profile, suitable for once-daily dosing in humans.

The first in-human study of the safety, tolerability, pharmacokinetics, and pharmacodynamics of abediterol at once-daily doses of $5,10,25$, or $50 \mu \mathrm{g}$ showed potent, rapid, and sustained bronchodilatory effects of this drug in healthy male subjects (Timmer et al., 2014; Montuschi and Ciabattoni, 2015). A dose-dependent increase of adverse events was observed, the most frequent being palpitations, tremor, nausea, and asthenia; most were mild in intensity and resolved without the need for intervention. This phase I study suggested that abediterol at measured doses is generally safe and well tolerated. It increases airway conductance and decreases airway resistance for up to 36 hours after a single dose, consistent with preclinical data. In a subsequent phase II study conducted in 25 male patients with mild-to-moderate persistent asthma, all doses of abediterol demonstrated statistically significant improvements in lung function compared with both placebo and salmeterol (Beier et al., 2014). To further evaluate lower abediterol doses, in a second phase II study, abediterol $0.313,0.625,1.25$, and $2.5 \mu \mathrm{g}$ doses were evaluated and compared with salbutamol and placebo in male and female patients with persistent asthma (Singh et al., 2014). Patients who received abediterol at these lower doses experienced rapid and sustained bronchodilation, with statistically significant benefits compared with placebo and a magnitude of effect comparable with salbutamol for all but the lowest abediterol dose. This result is also consistent with a later phase II study assessing the efficacy, safety, and tolerability of abediterol at $2.5,5$, and $10 \mu \mathrm{g}$ doses, given once daily for 7 days in patients with stable, persistent asthma (Beier et al., 2017). These results

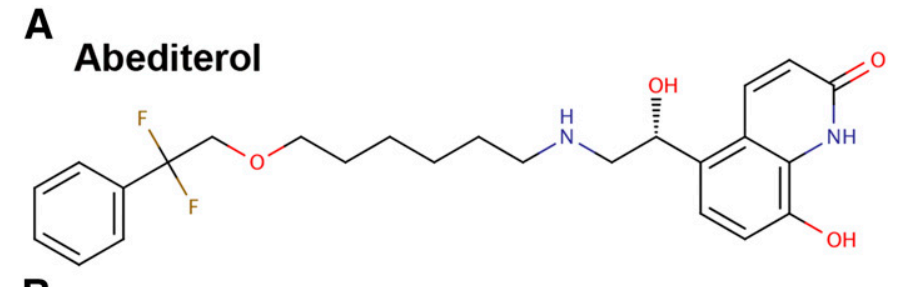

B

\section{Bedoradrine}

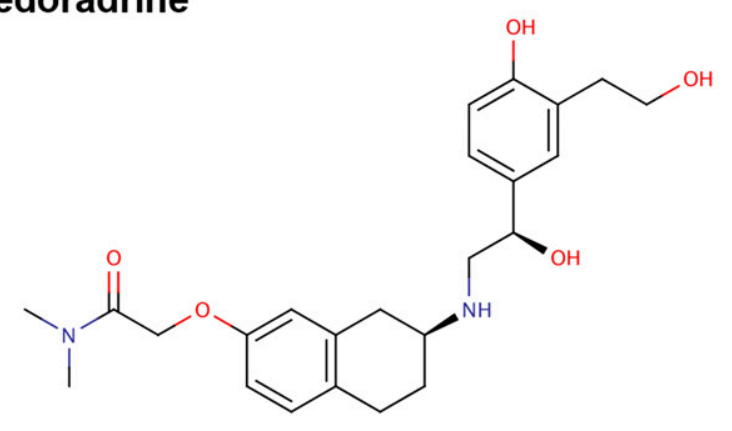

Fig. 8. Chemical structures of two potential asthma drugs acting on $\beta_{2} \mathrm{AR}$ : (A) abediterol, (B) bedoradrine.

suggest that abediterol is a promising new once-daily LABA for the treatment of asthma and COPD. The results of the conducted phase III studies are not available at this time.

2. Bedoradrine. Bedoradrine (KUR-1246, MN221) is a novel $\beta_{2}$-agonist with a chemical structure similar to other classic $\beta_{2}$-agonists. It has a tetrahydronaphthol substitution in the terminal amino group of the ethylamine side chain. It is similar to other LABAs by having a catechol-like group as the head and an ethanolamine group as the middle moiety. The remarkable characteristic of bedoradrine is the tetrahydronaphtyl substitution in the terminal amino group, which is believed to be essential for the potent activity and high selectivity of bedoradrine over other $\beta$-adrenergic receptor subtypes (Kiguchi et al., 2002).

Bedoradrine was initially developed as a tocolytic agent. In vitro receptor binding and cell activation studies in animals have demonstrated that bedoradrine has a high selectivity for $\beta_{2}$ over other $\beta$-adrenergic receptor subtypes (Kobayashi et al., 2001; Inoue et al., 2009). Later, bedoradrine entered in clinical development for the treatment of acute asthma exacerbations via intravenous delivery. Two phase I clinical studies (ClinicalTrials.gov Identifier: NCT01013142, NCT01551316) evaluated the safety, tolerability pharmacokinetics, and preliminary bronchodilator efficacy of bedoradrine in patients with COPD. Two subsequent phase II studies assessed the safety and efficacy of bedoradrine in escalating doses and a fixed dose in patients with mild-to-moderate asthma and moderateto-severe asthma, respectively (Matsuda et al., 2012). Another randomized, placebo-controlled phase II study evaluated the safety and efficacy of intravenous bedoradrine added to the standard therapy in patients with 
asthma exacerbations (House et al., 2015). It is still unclear whether intravenous bedoradrine can significantly improve lung function in severe, acute asthma exacerbations in addition to standard therapy; however, its use was associated with significantly improved dyspnea scores (Matsuda et al., 2012; House et al., 2015). Results from these studies demonstrated that bedoradrine is safe and without significant adverse effects in bedoradrine-treated patients (Matsuda et al., 2012; Antoniu, 2014). The current safety profile of bedoradrine and the potential for increased selectivity and reduced side effects in humans, as well as the intravenous route of administration, support further clinical development of bedoradrine as a novel therapy for asthma.

\section{Future Perspectives}

Although in most patients asthma can be well controlled with current available therapeutics, there are still a large number of asthma patients who require more effective therapies. Our new understanding of the heterogeneity of asthma also suggests the need for developing novel therapeutics for certain types of asthma, including the nonatopic, $\mathrm{T}_{\mathrm{H}} 2$ low, or obesityrelated asthma endotypes. Despite such demand, drug development in the past two decades for asthma has been slow. The current mainstream treatment methods for asthma, which include the combined use of ICSs, LABAs, and LAMAs, were established more than 15 years ago. Biologic drugs such as antibodies targeting IL-5 and IgE were introduced as a new class of anti-inflammatory therapeutics recently, which provided additional add-on options for asthma patients with certain phenotypes (Pelaia et al., 2012). Biologics are also under development for the inhibition of IL-13, IL-4, and other cytokines.

In this study, we will review GPCR-targeting ligands that have potential as new asthma drugs, with a focus on those that have entered clinical trials (Fig. 9). However, we would also like to point out that there are a number of other promising GPCR modulators in the early development for asthma. Those include bitter taste receptor agonists such as saccharin, chloroquine, and quinine (Deshpande et al., 2010; Grassin-Delyle et al., 2013); calcium-sensing receptor antagonists (Yarova et al., 2015); and specialized proresolving lipid mediators (SPMs) (Barnig and Levy, 2015). They may as well offer exciting opportunities for the development of novel asthma therapeutics (Pera and Penn, 2016). In addition, we will also review new opportunities in GPCR drug development, including the structure-based drug design and the development of GPCR-targeting antibodies.

\section{A. G Protein-Coupled Receptor Drugs in Early Stage of Development}

1. AZD8871 as a Dual-Action Ligand of Muscarinic Receptors and $\beta_{2}$-Adrenergic Receptor. Dual-action therapeutic methods combining LAMAs and LABAs have

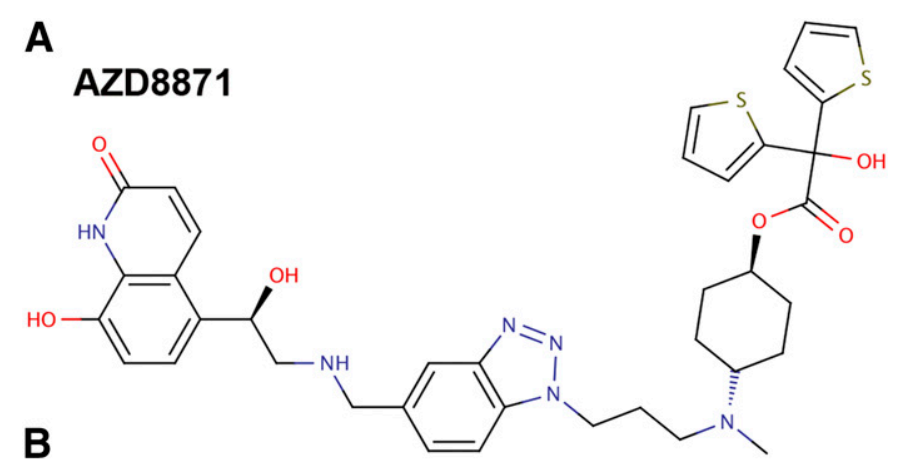

CVT-6883

C

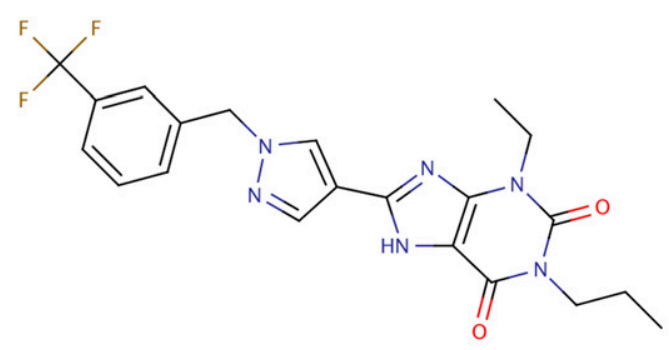

Toreforant

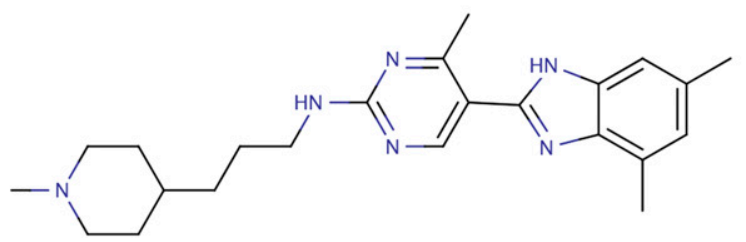

Fig. 9. Chemical structures of three GPCR-targeting ligands that are in early stage of development for asthma. (A) AZD8871, (B) CVT-6883, and (C) toreforant.

been introduced to achieve better efficacy compared with monotherapy with either class alone (Rolla and Brussino, 2018). Bifunctional molecules that represent a fusion of established LAMAs and LABAs in one molecule by an inactive spine are known as muscarinic antagonists $/ \beta_{2}$-agonists (MABAs). Several MABAs, such as batefenterol (GSK961081), AZD2115, and AZD8871, are currently in clinical development, aiming to achieve a synergy between the pharmacological actions of LAMAs and LABAs. MABAs are also expected to provide a potentially simpler technical and clinical development pathway compared with the dual therapy (Bateman et al., 2013; Page and Cazzola, 2014; Gross and Barnes, 2017).

Although MABAs were designed to occupy the orthosteric sites in $\beta_{2} \mathrm{AR}$ and MRs, their pharmacological action may be more complex. Pharmacological studies on MABA THRX-198321 suggested that the muscarinic antagonist moiety may occupy an allosteric site in $\beta_{2} \mathrm{AR}$ and the $\beta_{2}$-agonist moiety may occupy the allosteric site in MRs (Steinfeld et al., 2011). The exosite in the extracellular vestibule of $\beta_{2} \mathrm{AR}$ (Masureel et al., 2018) could be the allosteric site for THRX-198321. Therefore, THRX-198321 acts as a bivalent ligand, not just a bifunctional ligand, on $\beta_{2} \mathrm{AR}$ and MRs in a way that it 
occupies both orhosteric and allosteric sites. As a result, its affinities are higher at both receptors relative to the monovalent fragments. Whether other MABAs exhibit similar pharmacological properties needs further investigation.

AZD8871 (LAS191351) is an inhaled long-acting MABA under development for the treatment of COPD and asthma. To date, several phase I and II clinical studies have been completed with AZD8871 on COPD subjects (Singh et al., 2019), whereas the first phase I clinical trial for asthma was recently completed in 2018 (ClinicalTrials.gov Identifier: NCT02573155). This particular trial was a randomized, placebo-controlled two-part study to assess the safety, tolerability, pharmacokinetics, and pharmacodynamics of AZD8871 delivered by inhalation in asthmatic and COPD subjects. In conclusion, AZD8871 was safe and well tolerated, and it showed sustained bronchodilation at different doses. It delivered clinically meaningful improvement of symptoms and was superior to the reference agents, indacaterol (ultra-LABA) and tiotropium (LAMA), at a high dose $(1800 \mu \mathrm{g})$. Further clinical development of AZD8871 in larger studies is required to evaluate its potential as a novel treatment option for asthma and COPD.

2. CVT-6883 as an Antagonist of Adenosine A2B Receptor. From the 1980s, adenosine has been found to induce bronchoconstriction in patients with asthma and to increase the concentrations of proinflammatory mediators released from mast cells such as histamine, tryptase, $\mathrm{LTC}_{4}$, and $\mathrm{PGD}_{2}$ (Cushley et al., 1983; Crimi et al., 1997). This suggested that adenosine may cause bronchoconstriction through mast cell activation (Polosa, 2002; Holgate, 2005). In addition, higher concentrations of adenosine have been detected in the bronchoalveolar lavage fluid and the exhaled breath condensate of patients with asthma (Driver et al., 1993; Huszár et al., 2002). This suggested that adenosine may also function as a paracrine mediator for the inflammatory responses in the lung.

The effects of adenosine are mediated through a group of class A GPCRs comprising four subtypes, named as $\mathrm{A}_{1}, \mathrm{~A}_{2 \mathrm{~A}}, \mathrm{~A}_{2 \mathrm{~B}}$, and $\mathrm{A}_{3}$ adenosine receptors (Olah and Stiles, 1995). The $A_{2 B}$ receptor has been suggested to play an important role in the pathophysiology of asthma by mediating airway reactivity and modulating chronic inflammatory responses in the lung, which can be inhibited by selective antagonists of the $\mathrm{A}_{2 \mathrm{~B}}$ receptor (Feoktistov and Biaggioni, 1995; Feoktistov et al., 2001; Ryzhov et al., 2004; Zhong et al., 2004, 2005).

The compound CVT-6883 is a potent and selective $\mathrm{A}_{2 \mathrm{~B}}$ receptor antagonist. It has a much greater affinity for the $\mathrm{A}_{2 \mathrm{~B}}$ receptor $(\mathrm{Ki}=22 \mathrm{nM})$ than for other adenosine receptors (Elzein et al., 2008). In animal studies, CVT6883 was found to be as effective as monteleukast as it decreased the number of inflammatory cells in bronchoalveolar lavage fluid and the production of macrophage-derived proinflammatory mediators (Fan and Mustafa, 2002; Fan et al., 2003; Fan and Jamal Mustafa, 2006; Sun et al., 2006; Mustafa et al., 2007). In the subsequent phase I clinical trials, CVT-6883 was demonstrated to be safe, well tolerated, and sustainable at a once-daily chronic dosage (Kalla and Zablocki, 2009). It may provide a new therapeutic option for several disease areas, including asthma, COPD, and pulmonary fibrosis.

3. Toreforant as an Antagonist of Histamine H4 Receptor. Histamine has long been suspected to play a role in the pathogenesis of asthma. Among the four subtypes of histamine receptors (Seifert et al., 2013), the histamine 4 receptor (H4R) is a chemotactic receptor expressed on hematopoietic cells, mast cells, and DCs (O'Reilly et al., 2002; Buckland et al., 2003; Hofstra et al., 2003; Gutzmer et al., 2005; Damaj et al., 2007; Bäumer et al., 2008; Gschwandtner et al., 2011; Seifert et al., 2013; Jemima et al., 2014). The results of studies testing selective H4R antagonists in animal models of asthma suggested that H4R plays a role in the pathophysiology of asthma by mediating lung function and inflammation (Neumann et al., 2014; Thurmond, 2015).

Toreforant is a potent and selective H4R antagonist with a Ki of $8.4 \pm 2.2 \mathrm{nM}$. The affinity for human H4R is 25 -fold higher than that for histamine 3 receptor, and there was no detectable affinity for histamine 1 receptor or histamine 2 receptor. Toreforant has been found to be an anti-inflammatory reagent in mouse models of asthma and arthritis. In phase I clinical studies, toreforant was safe and well tolerated in healthy subjects (Thurmond et al., 2017). However, toreforant failed to provide therapeutic benefit in a recent phase II clinical study involving patients with eosinophilic asthma (Kollmeier et al., 2018), which has brought doubts to the use of H4R antagonists in the treatment of asthma.

4. FX125L. The compound FX125L was developed as a new anti-inflammatory reagent. It has gone through phase I trials and entered phase II clinical trials for the treatment of asthma and other inflammatory diseases (impact.ref.ac.uk/casestudies/CaseStudy.aspx?Id=7262). All research results on FX125L and its molecular modifications have been published as patents (patents.google.com/patent/US20090036486). The biologic target of this compound was proposed as a class A GPCR - the somatostatin receptor subtype 2, which still remains unsettled.

\section{B. New Opportunities in G Protein-Coupled Receptor Drug Development for Asthma}

GPCRs play important roles in asthma pathophysiology, which is evidenced by the wide use of GPCRtargeting drugs in the clinic and by the number of GPCR-targeting drug candidates in clinical development. Currently, significant research efforts are directed toward a better understanding of asthma pathophysiology, which may lead to the identification 
Wendell et al.

of new GPCR targets and novel therapeutic methods. As for drug development, recent progress in the GPCR pharmacology and structural biology provided new opportunities. Structure-based drug design has emerged as a powerful method for developing novel GPCR compounds with desired properties. GPCR-targeting therapeutic antibodies also represent a new frontier in GPCR drug discovery.

1. New Targets and Novel Therapeutic Methods. There are still significant research efforts directed at identifying new GPCR targets for the treatment of asthma, especially for the endotype-guided targeted asthma therapy. A recent collaborative study from multiple research centers in the United States reported that a GPCR, the FPR2/lipoxin $\mathrm{A}_{4}$ receptor (ALX), and its ligands define a new endotype for severe asthma (Ricklefs et al., 2017). FPR2/ALX exhibits unusual functional promiscuity by recognizing chemically and functionally distinct ligands to trigger different signaling pathways (Prevete et al., 2015; He and Ye, 2017; Weiß and Kretschmer, 2018; Raabe et al., 2019). It has been proposed to function as a checkpoint receptor that responds to ligands generated at different stages of inflammation to promote or resolve inflamation to keep a balanced inflammatory process. Antagonists or biased agonists of FPR2/ALX may be developed as potential therapeutics for the particular asthma endotype associated with imbalanced FPR2/ALX signaling (Raabe et al., 2019). Also, the currently used leukotriene receptor antagonists only target CysLTR $_{1}$ and block the signaling of $\mathrm{LTC}_{4}$ and $\mathrm{LTD}_{4}$, but not $\mathrm{LTE}_{4}$. $\mathrm{LTE}_{4}$ is a potent proinflammatory mediator in the airways. However, the exact receptor for $\mathrm{LTE}_{4}$ is still in debate (Paruchuri et al., 2009; Kanaoka et al., 2013). Identification of $\mathrm{LTE}_{4}$ receptor will allow the development of new antagonists, which may provide additional therapeutic benefits on top of current leukotriene receptor antagonists (Austen et al., 2009; Kanaoka and Boyce, 2014).

Identifying novel targets for asthma and the subsequent drug development is a long, ongoing effort. In the near future, novel therapeutic methods may emerge through repurposing of currently used GPCR drugs. As discussed previously, blocking detrimental $\beta_{2} \mathrm{AR}$ signaling by $\beta$-blockers has been shown to reduce lung inflammation and receptor desensitization associated with chronic use of LABAs, although the potential side effect of bronchoconstriction is a concern. In this regard, $\beta_{2} \mathrm{AR}$ negative allosteric modulators may be useful because they only dampen, not completely block, the signaling of $\beta_{2} \mathrm{AR}$. The therapeutic benefits of tiotropium as a LAMA also need to be further determined. Current asthma treatment guidelines only recommend tiotropium as an add-on therapy for severe asthma poorly controlled with LABA/ICS. However, it is likely that tiotropium may offer therapeutic benefits for a boutique group of asthma patients. Studies suggested its therapeutic potential for pediatric asthma patients (Kerstjens et al., 2016; Goldstein, 2019). Also, it may offer an alternative option if LABAs are not useful due to irresponsiveness or severe side effects (Buhl et al., 2018). Additional clinical studies are needed to test these ideas and clarify controversies. Continuous research into the mechanisms of GPCR signaling in asthma will bring more opportunities in drug repurposing for the treatment of asthma.

2. Structure-Based Drug Design. The successful determination of GPCR structures with various drugs allows us to understand the drug action at a molecular level. These structures may serve as the basis of structure-based drug design (also known as rational drug design) to assist in the development of new drugs. Structure-based drug design is a crucial tool in preclinical drug development and has led to the delivery of several successful drugs such as Crixivan for treating AIDS (Chen et al., 2003), Tamiflu for treating influenza (Lew et al., 2000), Gleevec for treating leukemia (Nagar et al., 2002), and Zelboraf for treating melanoma (Blundell, 2017). Compounds that have been identified through the structure-based methods using recently solved GPCR crystal structures are now progressing into clinical trials (Congreve et al., 2012; Bach et al., 2013; Cheng et al., 2017). In the absence of crystal structures, homology modeling is the most accurate and practical tool for the predictions of new GPCR structures. The predicted homology models can yield comparable success rates in structure-based drug design with respect to the corresponding experimentally solved structures, especially for those that have been through refinement/selection with experimental data (Carlsson et al., 2011; Langmead et al., 2012; Mysinger et al., 2012; Lim et al., 2018). Compounds that have been discovered through structure-based drug design using GPCR homology models have also entered clinical trials (Langmead et al., 2012; Huang et al., 2018). Given the continuous rise of experimentally solved GPCR crystal structures, which also facilitate the generation of accurate homology structural models with chemically diverse ligands, it is anticipated that structure-based drug design methods will contribute increasingly to the GPCR drug development efforts, including those for asthma, to develop novel therapeutics with more desirable pharmacological properties.

3. G Protein-Coupled Receptor Antibodies as Therapeutics. Although small molecules have been successfully used to target and modulate GPCRs in the treatment of many diseases, selectivity and potency are still a major challenge in GPCR drug discovery (Martí-Solano et al., 2016; Hauser et al., 2017; Latorraca et al., 2017; Insel et al., 2018). In comparison with small molecules, GPCR antibodies may offer better specificity (e.g., by stabilizing particular conformational states of the highly dynamic receptors), restricted central nervous system penetration, new ways of 
regulating receptor signaling (e.g., reducing signaling by causing receptor internalization), and prolonged in vivo action (Hutchings et al., 2017).

Developing therapeutic antibodies for GPCRs is challenging due to many factors such as difficulties in immunization and limited surface areas in GPCRs for antibody recognition (Hutchings et al., 2017). To date, only two GPCR-targeting antibodies have been approved by FDA, contrasting the fact that more than $30 \%$ of small-molecule drugs target GPCRs (Santos et al., 2017). One of them is mogamulizumab targeting CCR4 (Antoniu, 2010; Yu et al., 2017; Kim et al., 2018), and the other one is erenumab targeting calcitonin gene-related receptor (Goadsby et al., 2017; Garland et al., 2019). Mogamulizumab and erenumab were approved by FDA in 2018 for the treatment of relapsed or refractory mycosis fungoides and Sézary disease, and the prevention of migraine, respectively. Mogamulizumab was also in phase I studies for the management/ treatment of asthma (Pease and Horuk, 2014). Multiple GPCR-targeting antibodies are currently in clinical trials or preclinical development (Hutchings et al., 2017). One of them is a DP2-targeting antibody, which could deplete inflammatory cells, including eosinophils, ILC2, and $\mathrm{T}_{\mathrm{H}} 2$ cells, and therefore may present a novel therapy for asthma (Huang et al., 2016b). Preclinical studies indicated a low risk of central nervous system or gastrointestinal toxicity associated with this antibody (Rajapaksa et al., 2016).

One of the new avenues in antibody-based therapy is the development of single-chain antibodies, or nanobodies (Joost and Kolkman, 2010; Muyldermans, 2013; Steeland et al., 2016). Therapeutic nanobodies are derived from the heavy chain-only antibodies found in camelids. They are naturally occurring antibodies. In comparison with conventional antibodies, nanobodies are associated with several advantages, such as better solubility and tissue penetration, low immunogenicity, and high physical stability, and they can be easily produced in prokaryotic or eukaryotic host organisms (Harmsen and De Haard, 2007; Joost and Kolkman, 2010; Klarenbeek et al., 2012). Currently, only one nanobody, caplacizumab, is on the market (Elverdi and Eskazan, 2019). Caplacizumab targets the von Willebrand factor (a clotting protein), and it was approved by FDA in 2019 for the treatment of acquired thrombotic thrombocytopenic turpura and thrombosis (Kaplon and Reichert, 2018, 2019). Therapeutic nanobodies that target GPCRs are under development, including those for the chemokine(-like) receptors CXCR4, CXCR7, CXCR2, US28, and ChemR23 (Jähnichen et al., 2010; Maussang et al., 2013; Bradley et al., 2015; Peyrassol et al., 2016; de Wit et al., 2017; Heukers et al., 2018), as well as the metabotropic glutamate receptor 2 (Scholler et al., 2017) and protease-activated receptor 2 (PAR2) (Arizmendi et al., 2011; Pera and Penn, 2016). GPCRtargeting nanobodies have the potential to be developed as research tools, diagnostic tools, and medications for many diseases, including immune diseases, cancer, and chronic inflammatory diseases such as COPD and asthma (De Groof et al., 2019).

Nanobody-based therapeutics may be particularly suitable for treating asthma and other pulmonary diseases. First, due to the unique physical properties of nanobodies such as small sizes and high solubility and stability, they can be delivered through inhalation to the lung, providing a marked advantage in drug delivery (Van Heeke et al., 2017). Second, asthma is a complex inflammatory disease with multiple inflammatory mediators and pathways contributing to the pathogenesis of disease. The currently available antibody-based biologic agents only recognize a particular target, such as IgE or the IL-5 receptor, thus limiting their therapeutic efficacy. Because of their small size, nanobodies can be easily linked together or to other molecules as divalent or multivalent antibodies that have multiple targets (Steeland et al., 2016). Multivalent nanobodies that can recognize different inflammatory mediators simultaneously may offer a better control of lung inflammation by blocking multiple inflammatory pathways.

\section{Authorship Contributions \\ Wrote or contributed to the writing of the manuscript: Wendell, Fan, Zhang.}

\section{References}

Abraham WM, Ahmed A, Cortes A, Sielczak M, and Hallmon J (1993) The effects of a cysteinyl leukotriene antagonist (ONO-1078) on antigen-induced responses in allergic sheep. Prostaglandins Leukot Essent Fatty Acids 48:233-239.

Adcock IM, Caramori G, and Chung KF (2008) New targets for drug development in asthma. Lancet 372:1073-1087.

Alagha K, Palot A, Sofalvi T, Pahus L, Gouitaa M, Tummino C, Martinez S, Charpin D, Bourdin A, and Chanez P (2014) Long-acting muscarinic receptor antagonists for the treatment of chronic airway diseases. Ther Adv Chronic Dis 5:85-98.

Albertson TE, Sutter ME, and Chan AL (2015) The acute management of asthma. Clin Rev Allergy Immunol 48:114-125.

Anderson GP (2008) Endotyping asthma: new insights into key pathogenic mechanisms in a complex, heterogeneous disease. Lancet 372:1107-1119.

Anderson GP, Lindén A, and Rabe KF (1994) Why are long-acting beta-adrenoceptor agonists long-acting? Eur Respir J 7:569-578.

Anderson P, Lötvall J, and Lindén A (1996) Relaxation kinetics of formoterol and salmeterol in the guinea pig trachea in vitro. Hai 174:159-170.

Anderson SD (2004) Single-dose agents in the prevention of exercise-induced asthma: a descriptive review. Treat Respir Med 3:365-379.

Angeli V, Staumont D, Charbonnier AS, Hammad H, Gosset P, Pichavant M, Lambrecht BN, Capron M, Dombrowicz D, and Trottein F (2004) Activation of the D prostanoid receptor 1 regulates immune and skin allergic responses. J Immunol 172:3822-3829.

Antoniu S (2014) Bedoradrine for treating asthma and chronic obstructive pulmonary disease. Expert Opin Investig Drugs 23:1149-1156.

Antoniu SA (2010) Mogamulizumab, a humanized mAb against C-C chemokine receptor 4 for the potential treatment of T-cell lymphomas and asthma. Curr Opin Mol Ther 12:770-779.

Aparici M, Gómez-Angelats M, Vilella D, Otal R, Carcasona C, Viñals M, Ramos I, Gavaldà A, De Alba J, Gras J, et al. (2012) Pharmacological characterization of abediterol, a novel inhaled $\beta(2)$-adrenoceptor agonist with long duration of action and a favorable safety profile in preclinical models. J Pharmacol Exp Ther 342: 497-509.

Arai I, Takano N, Hashimoto Y, Futaki N, Sugimoto M, Takahashi N, Inoue T, and Nakaike S (2004) Prostanoid DP1 receptor agonist inhibits the pruritic activity in NC/Nga mice with atopic dermatitis. Eur J Pharmacol 505:229-235.

Arboe B and Ulrik CS (2013) Beta-blockers: friend or foe in asthma? Int J Gen Med 6: $549-555$.

Arcoleo F, Milano S, D'Agostino P, and Cillari E (1995) Effect of exogenous leukotriene B4 (LTB4) on BALB/c mice splenocyte production of Th1 and Th2 lymphokines. Int $J$ Immunopharmacol 17:457-463.

Arizmendi NG, Abel M, Mihara K, Davidson C, Polley D, Nadeem A, El Mays T, Gilmore BF, Walker B, Gordon JR, et al. (2011) Mucosal allergic sensitization to cockroach allergens is dependent on proteinase activity and proteinase-activated receptor-2 activation. J Immunol 186:3164-3172. 
Arm JP, O'Hickey SP, Hawksworth RJ, Fong CY, Crea AE, Spur BW, and Lee TH (1990) Asthmatic airways have a disproportionate hyperresponsiveness to LTE4, as compared with normal airways, but not to LTC4, LTD4, methacholine, and histamine. Am Rev Respir Dis 142:1112-1118.

Augstein J, Farmer JB, Lee TB, Sheard P, and Tattersall ML (1973) Selective inhibitor of slow reacting substance of anaphylaxis. Nat New Biol 245:215-217.

Aun MV, Bonamichi-Santos R, Arantes-Costa FM, Kalil J, and Giavina-Bianchi P (2017) Animal models of asthma: utility and limitations. J Asthma Allergy 10: 293-301.

Austen KF, Maekawa A, Kanaoka Y, and Boyce JA (2009) The leukotriene E4 puzzle: finding the missing pieces and revealing the pathobiologic implications. J Allergy Clin Immunol 124:406-414, quiz 415-416.

Bach MK, Brashler JR, and Gorman RR (1977) On the structure of slow reacting substance of anaphylaxis: evidence of biosynthesis from arachidonic acid. Prostaglandins 14:21-38.

Bach P, Antonsson T, Bylund R, Björkman JA, Österlund K, Giordanetto F, van Giezen JJ, Andersen SM, Zachrisson H, and Zetterberg F (2013) Lead optimization of ethyl 6-aminonicotinate acyl sulfonamides as antagonists of the P2Y12 receptor: separation of the antithrombotic effect and bleeding for candidate drug AZD1283. $J$ Med Chem 56:7015-7024.

Baker JG (2005) The selectivity of beta-adrenoceptor antagonists at the human beta1, beta2 and beta3 adrenoceptors. Br J Pharmacol 144:317-322.

Baker JG (2010) The selectivity of beta-adrenoceptor agonists at human beta1-, beta2- and beta3-adrenoceptors. Br J Pharmacol 160:1048-1061.

Baker JG, Proudman RG, and Hill SJ (2015) Salmeterol's extreme $\beta 2$ selectivity is due to residues in both extracellular loops and transmembrane domains. Mol Pharmacol 87:103-120.

Ball DI, Brittain RT, Coleman RA, Denyer LH, Jack D, Johnson M, Lunts LH, Nials AT, Sheldrick KE, and Skidmore IF (1991) Salmeterol, a novel, long-acting beta 2adrenoceptor agonist: characterization of pharmacological activity in vitro and in vivo. Br J Pharmacol 104:665-671.

Bandaru S, Marri VK, Kasera P, Kovuri P, Girdhar A, Mittal DR, Ikram S, Gv R, and Nayarisseri A (2014) Structure based virtual screening of ligands to identify cysteinyl leukotriene receptor 1 antagonist. Bioinformation 10:652-657.

Bandeira-Melo C, Hall JC, Penrose JF, and Weller PF (2002) Cysteinyl leukotrienes induce IL-4 release from cord blood-derived human eosinophils. J Allergy Clin Immunol 109:975-979.

Barnes N, Pavord I, Chuchalin A, Bell J, Hunter M, Lewis T, Parker D, Payton M, Collins LP, Pettipher R, et al. (2012) A randomized, double-blind, placebocontrolled study of the CRTH2 antagonist OC000459 in moderate persistent asthma. Clin Exp Allergy 42:38-48.

Barnes NC, de Jong B, and Miyamoto T (1997) Worldwide clinical experience with the first marketed leukotriene receptor antagonist. Chest 111 (Suppl):52S-60S.

Barnes NC and Pujet JC (1997) Pranlukast, a novel leukotriene receptor antagonist: results of the first European, placebo controlled, multicentre clinical study in asthma. Thorax 52:523-527.

Barnes PJ (1993) Muscarinic receptor subtypes in airways. Eur Respir J 6:328-331.

Barnes PJ (2006) Corticosteroid effects on cell signalling. Eur Respir J 27:413-426.

Barnes PJ (2010a) Inhaled corticosteroids. Pharmaceuticals (Basel) 3:514-540.

Barnes PJ (2010b) New therapies for asthma: is there any progress? Trends Pharmacol Sci 31:335-343.

Barnes PJ (2013) Theophylline. Am J Respir Crit Care Med 188:901-906.

Barnig C and Levy BD (2015) Innate immunity is a key factor for the resolution of inflammation in asthma. Eur Respir Rev 24:141-153.

Barton AK and Gehlen H (2016) Pulmonary remodeling in equine asthma: what do we know about mediators of inflammation in the horse? Mediators Inflamm 2016: 5693205 .

Bateman ED, Guerreros AG, Brockhaus F, Holzhauer B, Pethe A, Kay RA, and Townley RG (2017) Fevipiprant, an oral prostaglandin $\mathrm{DP}_{2}$ receptor (CRTh2) antagonist, in allergic asthma uncontrolled on low-dose inhaled corticosteroids. Eur Respir J 50:1700670.

Bateman ED, Kornmann O, Ambery C, and Norris V (2013) Pharmacodynamics of GSK961081, a bi-functional molecule, in patients with COPD. Pulm Pharmacol Ther 26:581-587.

Bateman ED, O’Brien C, Rugman P, Luke S, Ivanov S, and Uddin M (2018) Efficacy and safety of the CRTh2 antagonist AZD1981 as add-on therapy to inhaled corticosteroids and long-acting $\beta_{2}$-agonists in patients with atopic asthma. Drug Des Devel Ther 12:1093-1106.

Bäumer W, Wendorff S, Gutzmer R, Werfel T, Dijkstra D, Chazot P, Stark H, and Kietzmann M (2008) Histamine $\mathrm{H} 4$ receptors modulate dendritic cell migration through skin--immunomodulatory role of histamine. Allergy 63:1387-1394.

Beasley R, Perrin K, Weatherall M, and Wijesinghe M (2010) Call for withdrawal of LABA single-therapy inhaler in asthma. Lancet 376:750-751.

Beavo JA and Brunton LL (2002) Cyclic nucleotide research--still expanding after half a century. Nat Rev Mol Cell Biol 3:710-718.

Beier J, Fuhr R, Massana E, Jiménez E, Seoane B, de Miquel G, and Ruiz S (2014) Abediterol (LAS100977), a novel long-acting $\beta 2$-agonist: efficacy, safety and tolerability in persistent asthma. Respir Med 108:1424-1429.

Beier J, Fuhr R, Seoane B, Massana E, de Miquel G, Pujol H, and Ruiz S (2017) Efficacy, safety, and tolerability of once-daily abediterol in patients with stable, persistent asthma: a Phase II, randomized, 7-day, crossover study. Pharmacol Res Perspect 5:e0356.

Beller TC, Maekawa A, Friend DS, Austen KF, and Kanaoka Y (2004) Targeted gene disruption reveals the role of the cysteinyl leukotriene 2 receptor in increased vascular permeability and in bleomycin-induced pulmonary fibrosis in mice. J Biol Chem 279:46129-46134.

Bellini A, Marini MA, Bianchetti L, Barczyk M, Schmidt M, and Mattoli S (2012) Interleukin (IL)-4, IL-13, and IL-17A differentially affect the profibrotic and proinflammatory functions of fibrocytes from asthmatic patients. Mucosal Immunol 5:140-149.
Bergendal A, Lindén A, Skoogh BE, Gerspacher M, Anderson GP, and Löfdahl CG (1996) Extent of salmeterol-mediated reassertion of relaxation in guinea-pig trachea pretreated with aliphatic side chain structural analogues. $\mathrm{Br} J$ Pharmacol 117:1009-1015.

Bernstein PR (1998) Chemistry and structure--activity relationships of leukotriene receptor antagonists. Am J Respir Crit Care Med 157:S220-S225; discussion S2256, S247-8.

Berry MA, Hargadon B, Shelley M, Parker D, Shaw DE, Green RH, Bradding P, Brightling CE, Wardlaw AJ, and Pavord ID (2006) Evidence of a role of tumor necrosis factor alpha in refractory asthma. $N$ Engl $J$ Med 354:697-708.

Billington CK and Penn RB (2002) m3 muscarinic acetylcholine receptor regulation in the airway. Am J Respir Cell Mol Biol 26:269-272.

Billington CK, Penn RB, and Hall IP (2017) $\beta_{2}$ agonists. Handb Exp Pharmacol 237: 23-40.

Bisgaard H and Groth S (1987) Bronchial effects of leukotriene D4 inhalation in normal human lung. Clin Sci (Lond) 72:585-592.

Bisgaard H, Skoner D, Boza ML, Tozzi CA, Newcomb K, Reiss TF, Knorr B, and Noonan G (2009) Safety and tolerability of montelukast in placebo-controlled pediatric studies and their open-label extensions. Pediatr Pulmonol 44:568-579.

Blundell TL (2017) Protein crystallography and drug discovery: recollections of knowledge exchange between academia and industry. IUCrJ 4:308-321.

Bochnowicz S and Underwood DC (1995) Dose-dependent mediation of leukotriene D4-induced airway microvascular leakage and bronchoconstriction in the guinea pig. Prostaglandins Leukot Essent Fatty Acids 52:403-411.

Bock A and Mohr K (2013) Dualsteric GPCR targeting and functional selectivity: the paradigmatic M(2) muscarinic acetylcholine receptor. Drug Discov Today Technol 10:e245-e252.

Bokoch MP, Zou Y, Rasmussen SG, Liu CW, Nygaard R, Rosenbaum DM, Fung JJ, Choi HJ, Thian FS, Kobilka TS, et al. (2010) Ligand-specific regulation of the extracellular surface of a G-protein-coupled receptor. Nature 463:108-112.

Bond RA (2014) The intrinsic bias of generalizations. Am J Respir Crit Care Med 189: 359.

Bouyssou T, Casarosa P, Naline E, Pestel S, Konetzki I, Devillier P, and Schnapp A (2010) Pharmacological characterization of olodaterol, a novel inhaled beta2 adrenoceptor agonist exerting a 24-hour-long duration of action in preclinical models. J Pharmacol Exp Ther 334:53-62.

Bradley ME, Dombrecht B, Manini J, Willis J, Vlerick D, De Taeye S, Van den Heede K, Roobrouck A, Grot E, Kent TC, et al. (2015) Potent and efficacious inhibition of CXCR2 signaling by biparatopic nanobodies combining two distinct modes of action. Mol Pharmacol 87:251-262.

Bradley SJ, Wiegman CH, Iglesias MM, Kong KC, Butcher AJ, Plouffe B, Goupil E, Bourgognon JM, Macedo-Hatch T, LeGouill C, et al. (2016) Mapping physiological $\mathrm{G}$ protein-coupled receptor signaling pathways reveals a role for receptor phosphorylation in airway contraction. Proc Natl Acad Sci USA 113:4524-4529.

Braido F, Corsico A, Rogkakou A, Ronzoni V, Baiardini I, and Canonica GW (2015) The relationship between allergen immunotherapy and omalizumab for treating asthma. Expert Rev Respir Med 9:129-134.

Brigden G, du Cros P, and Wong S (2016) Barriers to new drug development in respiratory disease. Eur Respir $J$ 47:356-357.

Bristow MR (2000) Beta-adrenergic receptor blockade in chronic heart failure. Cir culation 101:558-569.

Bryant DH and Rogers P (1990) Oxitropium bromide: an acute dose response study of a new anticholinergic drug in combination with fenoterol in asthma and chronic bronchitis. Pulm Pharmacol 3:55-58.

Buckland KF, Williams TJ, and Conroy DM (2003) Histamine induces cytoskeletal changes in human eosinophils via the $\mathrm{H}(4)$ receptor. $\mathrm{Br} J$ Pharmacol 140: 1117-1127.

Buels KS and Fryer AD (2012) Muscarinic receptor antagonists: effects on pulmonary function. Handb Exp Pharmacol 208:317-341.

Buels KS, Jacoby DB, and Fryer AD (2012) Non-bronchodilating mechanisms of tiotropium prevent airway hyperreactivity in a guinea-pig model of allergic asthma. Br J Pharmacol 165:1501-1514.

Buhl R, FitzGerald JM, and Busse WW (2018) Tiotropium add-on to inhaled corticosteroids versus addition of long-acting $\beta_{2}$-agonists for adults with asthma. Respir Med 143:82-90.

Buller S, Zlotos DP, Mohr K, and Ellis J (2002) Allosteric site on muscarinic acetylcholine receptors: a single amino acid in transmembrane region 7 is critical to the subtype selectivities of caracurine $\mathrm{V}$ derivatives and alkane-bisammonium ligands. Mol Pharmacol 61:160-168.

Busse W, Nelson H, Wolfe J, Kalberg C, Yancey SW, and Rickard KA (1999) Comparison of inhaled salmeterol and oral zafirlukast in patients with asthma. $J$ Allergy Clin Immunol 103:1075-1080.

Busse W, Raphael GD, Galant S, Kalberg C, Goode-Sellers S, Srebro S, Edwards L, and Rickard K; Fluticasone Proprionate Clinical Research Study Group (2001) Low-dose fluticasone propionate compared with montelukast for first-line treatment of persistent asthma: a randomized clinical trial. J Allergy Clin Immunol 107:461-468

Busse WW, Dahl R, Jenkins C, and Cruz AA (2016) Long-acting muscarinic antagonists: a potential add-on therapy in the treatment of asthma? Eur Respir Rev $\mathbf{2 5}$ $54-64$.

Busse WW, Wenzel SE, Meltzer EO, Kerwin EM, Liu MC, Zhang N, Chon Y, Budelsky AL, Lin J, and Lin SL (2013) Safety and efficacy of the prostaglandin D2 receptor antagonist AMG 853 in asthmatic patients. J Allergy Clin Immunol 131: 339-345.

Calbet M, Andrés M, Armengol C, Bravo M, Eichhorn P, López R, García-González V, Roberts R, and Miralpeix M (2016) Pharmacological characterization of CRTh2 antagonist LAS191859: long receptor residence time translates into long-lasting in vivo efficacy. Pharmacol Res 111:208-216.

Callaerts-Vegh Z, Evans KL, Dudekula N, Cuba D, Knoll BJ, Callaerts PF, Giles H, Shardonofsky FR, and Bond RA (2004) Effects of acute and chronic administration 
of beta-adrenoceptor ligands on airway function in a murine model of asthma. Proc Natl Acad Sci USA 101:4948-4953.

Calverley PM, Anderson JA, Celli B, Ferguson GT, Jenkins C, Jones PW, Yates JC, and Vestbo J; TORCH Investigators (2007) Salmeterol and fluticasone propionate and survival in chronic obstructive pulmonary disease. N Engl J Med 356:775-789.

Campo P, Rodriguez F, Sanchez-Garcia S, Barranco P, Quirce S, Perez-Frances C, Gomez-Torrijos E, Cardenas R, Olaguibel JM, and Delgado J; Severe Asthma Workgroup; SEAIC Asthma Committee (2013) Phenotypes and endotypes of uncontrolled severe asthma: new treatments. J Investig Allergol Clin Immunol 23 76-88; quiz $71 \mathrm{p}$ follow 88.

Canning BJ and Chou Y (2008) Using guinea pigs in studies relevant to asthma and COPD. Pulm Pharmacol Ther 21:702-720.

Capra V, Thompson MD, Sala A, Cole DE, Folco G, and Rovati GE (2007) Cysteinylleukotrienes and their receptors in asthma and other inflammatory diseases: critical update and emerging trends. Med Res Rev 27:469-527.

Carlsson J, Coleman RG, Setola V, Irwin JJ, Fan H, Schlessinger A, Sali A, Roth BL, and Shoichet BK (2011) Ligand discovery from a dopamine D3 receptor homology model and crystal structure. Nat Chem Biol 7:769-778.

Carter AA and Hill SJ (2005) Characterization of isoprenaline- and salmeterolstimulated interactions between beta2-adrenoceptors and beta-arrestin 2 using beta-galactosidase complementation in $\mathrm{C} 2 \mathrm{C} 12$ cells. J Pharmacol Exp Ther $\mathbf{3 1 5}$ 839-848.

Casaburi R, Mahler DA, Jones PW, Wanner A, San PG, ZuWallack RL, Menjoge SS, Serby CW, and Witek T Jr (2002) A long-term evaluation of once-daily inhaled tiotropium in chronic obstructive pulmonary disease. Eur Respir $J$ 19:217-224.

Casarosa P, Bouyssou T, Germeyer S, Schnapp A, Gantner F, and Pieper M (2009) Preclinical evaluation of long-acting muscarinic antagonists: comparison of tiotropium and investigational drugs. J Pharmacol Exp Ther 330:660-668.

Casarosa P, Kiechle T, Sieger P, Pieper M, and Gantner F (2010) The constitutive activity of the human muscarinic M3 receptor unmasks differences in the pharmacology of anticholinergics. J Pharmacol Exp Ther 333:201-209.

Castan L, Magnan A, and Bouchaud G (2017) Chemokine receptors in allergic diseases. Allergy 72:682-690.

Castro M, Corren J, Pavord ID, Maspero J, Wenzel S, Rabe KF, Busse WW, Ford L Sher L, FitzGerald JM, et al. (2018) Dupilumab efficacy and safety in moderate-tosevere uncontrolled asthma. N Engl J Med 378:2486-2496.

Caulfield MP (1993) Muscarinic receptors--characterization, coupling and function. Pharmacol Ther 58:319-379.

Caulfield MP and Birdsall NJ (1998) International Union of Pharmacology. XVII Classification of muscarinic acetylcholine receptors. Pharmacol Rev 50:279-290.

Cazzola M, Imperatore F, Salzillo A, Di Perna F, Calderaro F, Imperatore A and Matera MG (1998) Cardiac effects of formoterol and salmeterol in patient suffering from COPD with preexisting cardiac arrhythmias and hypoxemia. Chest 114:411-415.

Cazzola M, Matera MG, and Donner CF (2005) Inhaled beta2-adrenoceptor agonists: cardiovascular safety in patients with obstructive lung disease. Drugs 65 1595-1610

Cazzola M, Page CP, Calzetta L, and Matera MG (2012) Pharmacology and therapeutics of bronchodilators. Pharmacol Rev 64:450-504.

Cazzola M, Page CP, Rogliani P, and Matera MG (2013) $\beta 2$-agonist therapy in lung disease. Am J Respir Crit Care Med 187:690-696.

Chabra R and Gupta M (2019) Asthma, Allergic and Environmental, StatPearls Publishing, Treasure Island, FL.

Chang JE, Doherty TA, Baum R, and Broide D (2014) Prostaglandin D2 regulates human type 2 innate lymphoid cell chemotaxis. J Allergy Clin Immunol 133 899-901.e3.

Chapman DG, Tully JE, Nolin JD, Janssen-Heininger YM, and Irvin CG (2014) Animal models of allergic airways disease: where are we and where to next? $J$ Cell Biochem 115:2055-2064.

Charlton SJ (2009) Agonist efficacy and receptor desensitization: from partial truths to a fuller picture. Br J Pharmacol 158:165-168.

Chazan R (2001) [The role of IgE in allergic inflammation in asthma]. Wiad Lek $\mathbf{5 4}$ 337-343.

Chen R, Smith SG, Salter B, El-Gammal A, Oliveria JP, Obminski C, Watson R, O'Byrne PM, Gauvreau GM, and Sehmi R (2017) Allergen-induced increases in sputum levels of group 2 innate lymphoid cells in subjects with asthma. Am $J$ Respir Crit Care Med 196:700-712.

Chen X, Kempf DJ, Li L, Sham HL, Vasavanonda S, Wideburg NE, Saldivar A, Marsh KC, McDonald E, and Norbeck DW (2003) Synthesis and SAR studies of potent HIV protease inhibitors containing novel dimethylphenoxyl acetates as P2 ligands. Bioorg Med Chem Lett 13:3657-3660.

Cheng H, Leff JA, Amin R, Gertz BJ, De Smet M, Noonan N, Rogers JD, Malbecq W, Meisner D, and Somers G (1996) Pharmacokinetics, bioavailability, and safety of montelukast sodium (MK-0476) in healthy males and females. Pharm Res 13 445-448.

Cheng RKY, Fiez-Vandal C, Schlenker O, Edman K, Aggeler B, Brown DG, Brown GA, Cooke RM, Dumelin CE, Doré AS, et al. (2017) Structural insight into allosteric modulation of protease-activated receptor 2. Nature 545:112-115.

Cherezov V, Rosenbaum DM, Hanson MA, Rasmussen SG, Thian FS, Kobilka TS, Choi HJ, Kuhn P, Weis WI, Kobilka BK, et al. (2007) High-resolution crystal structure of an engineered human beta2-adrenergic $\mathrm{G}$ protein-coupled receptor. Science 318:1258-1265.

Chilvers ER, Batty IH, Barnes PJ, and Nahorski SR (1990) Formation of inositol polyphosphates in airway smooth muscle after muscarinic receptor stimulation. J Pharmacol Exp Ther 252:786-791.

Choby GW and Lee S (2015) Pharmacotherapy for the treatment of asthma: current treatment options and future directions. Int Forum Allergy Rhinol 5 (Suppl 1): S35-S40.

Chong LK, Chess-Williams R, and Peachell PT (2002) Pharmacological characterisation of the beta-adrenoceptor expressed by human lung mast cells. Eur J Pharmacol 437:1-7.
Christie PE, Tagari P, Ford-Hutchinson AW, Charlesson S, Chee P, Arm JP, and Lee TH (1991) Urinary leukotriene E4 concentrations increase after aspirin challenge in aspirin-sensitive asthmatic subjects. Am Rev Respir Dis 143:1025-1029.

Chung KF, Wenzel SE, Brozek JL, Bush A, Castro M, Sterk PJ, Adcock IM, Bateman $\mathrm{ED}, \mathrm{Bel} \mathrm{EH}, \mathrm{Bleecker} \mathrm{ER}$, et al. (2014) International ERS/ATS guidelines on definition, evaluation and treatment of severe asthma [published correction appears in Eur Respir J (2014) 43:1216]. Eur Respir J 43:343-373.

Clark AL and Mitchelson F (1976) The inhibitory effect of gallamine on muscarinic receptors. Br J Pharmacol 58:323-331.

Cockcroft DW and Sears MR (2013) Are inhaled longacting $\beta 2$ agonists detrimental to asthma? Lancet Respir Med 1:339-346.

Coffman RL and Hessel EM (2005) Nonhuman primate models of asthma. J Exp Med 201:1875-1879.

Coleman RA (2009) On the mechanism of the persistent action of salmeterol: what is the current position? $\mathrm{Br}$ J Pharmacol 158:180-182.

Coleman RA, Johnson M, Nials AT, and Vardey CJ (1996) Exosites: their current status, and their relevance to the duration of action of long-acting beta 2adrenoceptor agonists. Trends Pharmacol Sci 17:324-330.

Coleman RA and Sheldrick RL (1989) Prostanoid-induced contraction of human bronchial smooth muscle is mediated by TP-receptors. Br J Pharmacol 96:688-692.

Coles SJ, Neill KH, Reid LM, Austen KF, Nii Y, Corey EJ, and Lewis RA (1983) Effects of leukotrienes C4 and D4 on glycoprotein and lysozyme secretion by human bronchial mucosa. Prostaglandins 25:155-170.

Colice GL (2011) Emerging therapeutic options for asthma. Am J Manag Care 17 (Suppl 3):S82-S89.

Congreve M, Andrews SP, Doré AS, Hollenstein K, Hurrell E, Langmead CJ, Mason JS, Ng IW, Tehan B, Zhukov A, et al. (2012) Discovery of 1,2,4-triazine derivatives as adenosine $\mathrm{A}(2 \mathrm{~A})$ antagonists using structure based drug design. J Med Chem $\mathbf{5 5}$ 1898-1903.

Cooper PR, Kurten RC, Zhang J, Nicholls DJ, Dainty IA, and Panettieri RA (2011) Formoterol and salmeterol induce a similar degree of $\beta 2$-adrenoceptor tolerance in human small airways but via different mechanisms. Br J Pharmacol 163:521-532.

Coqueret O, Dugas B, Mencia-Huerta JM, and Braquet P (1995) Regulation of IgE production from human mononuclear cells by beta 2 -adrenoceptor agonists. Clin Exp Allergy 25:304-311.

Cowburn AS, Holgate ST, and Sampson AP (1999) IL-5 increases expression of 5lipoxygenase-activating protein and translocates 5-lipoxygenase to the nucleus in human blood eosinophils. J Immunol 163:456-465.

Crimi N, Polosa R, and Mistretta A (1997) Purine derivatives in the study of allergic inflammation in respiratory diseases. Allergy $\mathbf{5 2}$ (Suppl):48-54.

Cruz AA (2018) Tiotropium: from COPD to young children with asthma? Lancet Respir Med 6:80-82.

Cushley MJ, Tattersfield AE, and Holgate ST (1983) Inhaled adenosine and guanosine on airway resistance in normal and asthmatic subjects. $\mathrm{Br} J$ Clin Pharmacol 15:161-165.

Dahlén SE, Björk J, Hedqvist P, Arfors KE, Hammarström S, Lindgren JA and Samuelsson B (1981) Leukotrienes promote plasma leakage and leukocyte adhesion in postcapillary venules: in vivo effects with relevance to the acute inflammatory response. Proc Natl Acad Sci USA 78:3887-3891.

Dahlén SE, Hansson G, Hedqvist P, Björck T, Granström E, and Dahlén B (1983) Allergen challenge of lung tissue from asthmatics elicits bronchial contraction that correlates with the release of leukotrienes C4, D4, and E4. Proc Natl Acad Sci USA 80:1712-1716.

Dahlén SE, Hedqvist P, Hammarström S, and Samuelsson B (1980) Leukotrienes are potent constrictors of human bronchi. Nature 288:484-486.

Dahlén SE, Malmström K, Nizankowska E, Dahlén B, Kuna P, Kowalski M, Lumry WR, Picado C, Stevenson DD, Bousquet J, et al. (2002) Improvement of aspirinintolerant asthma by montelukast, a leukotriene antagonist: a randomized, doubleblind, placebo-controlled trial. Am J Respir Crit Care Med 165:9-14.

Damaj BB, Becerra CB, Esber HJ, Wen Y, and Maghazachi AA (2007) Functional expression of $\mathrm{H} 4$ histamine receptor in human natural killer cells, monocytes, and dendritic cells. $J$ Immunol 179:7907-7915.

D'Amato G (2006) Role of anti-IgE monoclonal antibody (omalizumab) in the treatment of bronchial asthma and allergic respiratory diseases. Eur J Pharmacol 533:302-307.

Dantzer JA and Wood RA (2018) The use of omalizumab in allergen immunotherapy. Clin Exp Allergy 48:232-240.

Davidson AB, Lee TH, Scanlon PD, Solway J, McFadden ER Jr, Ingram RH Jr, Corey EJ, Austen KF, and Drazen JM (1987) Bronchoconstrictor effects of leukotriene E4 in normal and asthmatic subjects. Am Rev Respir Dis 135:333-337.

De Groof TWM, Bobkov V, Heukers R, and Smit MJ (2019) Nanobodies: new avenues for imaging, stabilizing and modulating GPCRs. Mol Cell Endocrinol 484:15-24.

De Lepeleire I, Reiss TF, Rochette F, Botto A, Zhang J, Kundu S, and Decramer M (1997) Montelukast causes prolonged, potent leukotriene D4-receptor antagonism in the airways of patients with asthma. Clin Pharmacol Ther 61:83-92.

de Wit RH, Heukers R, Brink HJ, Arsova A, Maussang D, Cutolo P, Strubbe B, Vischer HF, Bachelerie F, and Smit MJ (2017) CXCR4-Specific Nanobodies as Potential Therapeutics for WHIM syndrome. J Pharmacol Exp Ther 363 (1):35-44, doi: 10.1124/jpet.117.242735 28768817.

Dennis EA and Norris PC (2015) Eicosanoid storm in infection and inflammation. Nat Rev Immunol 15:511-523.

Deshpande DA, Theriot BS, Penn RB, and Walker JK (2008) Beta-arrestins specifically constrain beta2-adrenergic receptor signaling and function in airway smooth muscle. FASEB $J$ 22:2134-2141.

Deshpande DA, Wang WC, McIlmoyle EL, Robinett KS, Schillinger RM, An SS, Sham JS, and Liggett SB (2010) Bitter taste receptors on airway smooth muscle bronchodilate by localized calcium signaling and reverse obstruction. Nat Med 16 1299-1304.

DeWire SM, Ahn S, Lefkowitz RJ, and Shenoy SK (2007) Beta-arrestins and cell signaling. Annu Rev Physiol 69:483-510.

Deykin A, Wechsler ME, Boushey HA, Chinchilli VM, Kunselman SJ, Craig TJ, DiMango E, Fahy JV, Kraft M, Leone F, et al.; National Heart, Lung, and Blood 
Institute's Asthma Clinical Research Network (2007) Combination therapy with a long-acting beta-agonist and a leukotriene antagonist in moderate asthma. Am $J$ Respir Crit Care Med 175:228-234.

Diamant Z and Sampson AP (1999) Anti-inflammatory mechanisms of leukotriene modulators. Clin Exp Allergy 29:1449-1453.

Diamant Z, Sidharta PN, Singh D, O'Connor BJ, Zuiker R, Leaker BR, Silkey M, and Dingemanse J (2014) Setipiprant, a selective CRTH2 antagonist, reduces allergen-induced airway responses in allergic asthmatics. Clin Exp Allergy 44: $1044-1052$

Dickey BF, Walker JK, Hanania NA, and Bond RA (2010) beta-Adrenoceptor inverse agonists in asthma. Curr Opin Pharmacol 10:254-259.

Digby GJ, Shirey JK, and Conn PJ (2010) Allosteric activators of muscarinic receptors as novel approaches for treatment of CNS disorders. Mol Biosyst 6:1345-1354. Disse B, Reichl R, Speck G, Traunecker W, Ludwig Rominger KL, and Hammer R (1993) Ba 679 BR, a novel long-acting anticholinergic bronchodilator. Life Sci 52: 537-544.

Dixon RA, Kobilka BK, Strader DJ, Benovic JL, Dohlman HG, Frielle T, Bolanowski MA, Bennett CD, Rands E, Diehl RE, et al. (1986) Cloning of the gene and cDNA for mammalian beta-adrenergic receptor and homology with rhodopsin. Nature 321:75-79.

Domingo C, Palomares O, Sandham DA, Erpenbeck VJ, and Altman P (2018) The prostaglandin $\mathrm{D}_{2}$ receptor 2 pathway in asthma: a key player in airway inflammation. Respir Res 19:189.

Donald DE, Samueloff SL, and Ferguson D (1967) Mechanisms of tachycardia caused by atropine in conscious dogs. Am J Physiol 212:901-910.

Donohue JF, Wise R, Busse WW, Garfinkel S, Zubek VB, Ghafouri M, Manuel RC, Schlenker-Herceg R, and Bleecker ER (2016) Efficacy and safety of ipratropium bromide/albuterol compared with albuterol in patients with moderate-to-severe asthma: a randomized controlled trial. BMC Pulm Med 16:65.

Dörje F, Wess J, Lambrecht G, Tacke R, Mutschler E, and Brann MR (1991) Antagonist binding profiles of five cloned human muscarinic receptor subtypes. $J$ Pharmacol Exp Ther 256:727-733.

Drake MT, Violin JD, Whalen EJ, Wisler JW, Shenoy SK, and Lefkowitz RJ (2008) Beta-arrestin-biased agonism at the beta2-adrenergic receptor. $J$ Biol Chem 283 : $5669-5676$

Drazen JM (1988) Comparative contractile responses to sulfidopeptide leukotrienes in normal and asthmatic human subjects. Ann N Y Acad Sci 524:289-297.

Drazen JM and Austen KF (1987) Leukotrienes and airway responses. Am Rev Respir Dis 136:985-998.

Driver AG, Kukoly CA, Ali S, and Mustafa SJ (1993) Adenosine in bronchoalveolar lavage fluid in asthma. Am Rev Respir Dis 148:91-97.

Dunn CJ and Goa KL (2001) Zafirlukast: an update of its pharmacology and therapeutic efficacy in asthma. Drugs 61:285-315.

Düringer C, Grundström G, Gürcan E, Dainty IA, Lawson M, Korn SH, Jerre A, Håkansson HF, Wieslander E, Fredriksson K, et al. (2009) Agonist-specific patterns of beta 2-adrenoceptor responses in human airway cells during prolonged exposure. Br $J$ Pharmacol 158:169-179.

Eder W, Ege MJ, and von Mutius E (2006) The asthma epidemic. N Engl J Med 355: $2226-2235$.

Edwards MR, Haas J, Panettieri RA Jr, Johnson M, and Johnston SL (2007) Corticosteroids and beta2 agonists differentially regulate rhinovirus-induced interleukin-6 via distinct Cis-acting elements. J Biol Chem 282:15366-15375.

Ellis JL and Undem BJ (1994) Role of cysteinyl-leukotrienes and histamine in mediating intrinsic tone in isolated human bronchi. Am J Respir Crit Care Med 149 $118-122$.

Elverdi T and Eskazan AE (2019) Caplacizumab as an emerging treatment option for acquired thrombotic thrombocytopenic purpura. Drug Des Devel Ther 13 1251-1258.

Elzein E, Kalla RV, Li X, Perry T, Gimbel A, Zeng D, Lustig D, Leung K, and Zablocki $\mathrm{J}$ (2008) Discovery of a novel A2B adenosine receptor antagonist as a clinical candidate for chronic inflammatory airway diseases. $J$ Med Chem 51:2267-2278.

Erpenbeck VJ, Popov TA, Miller D, Weinstein SF, Spector S, Magnusson B, Osuntokun W, Goldsmith P, Weiss M, and Beier J (2016) The oral CRTh2 antagonist QAW039 (fevipiprant): a phase II study in uncontrolled allergic asthma. Pulm Pharmacol Ther 39:54-63.

Espinosa K, Bossé Y, Stankova J, and Rola-Pleszczynski M (2003) CysLT1 receptor upregulation by TGF-beta and IL-13 is associated with bronchial smooth muscle cell proliferation in response to LTD4. J Allergy Clin Immunol 111:1032-1040.

Fahy JV (2015) Type 2 inflammation in asthma--present in most, absent in many. Nat Rev Immunol 15:57-65.

Fajt ML, Gelhaus SL, Freeman B, Uvalle CE, Trudeau JB, Holguin F, and Wenzel SE (2013) Prostaglandin $D_{2}$ pathway upregulation: relation to asthma severity, control, and TH2 inflammation. J Allergy Clin Immunol 131:1504-1512.

Fajt ML and Wenzel SE (2017) Development of new therapies for severe asthma. Allergy Asthma Immunol Res 9:3-14.

Fallon PG, Ballantyne SJ, Mangan NE, Barlow JL, Dasvarma A, Hewett DR, McIlgorm A, Jolin HE, and McKenzie AN (2006) Identification of an interleukin (IL)-25dependent cell population that provides IL-4, IL-5, and IL-13 at the onset of helminth expulsion. $J$ Exp Med 203:1105-1116.

Fan M and Jamal Mustafa S (2006) Role of adenosine in airway inflammation in an allergic mouse model of asthma. Int Immunopharmacol 6:36-45.

Fan M and Mustafa SJ (2002) Adenosine-mediated bronchoconstriction and lung inflammation in an allergic mouse model. Pulm Pharmacol Ther 15:147-155.

Fan M, Qin W, and Mustafa SJ (2003) Characterization of adenosine receptor(s) involved in adenosine-induced bronchoconstriction in an allergic mouse model. Am $J$ Physiol Lung Cell Mol Physiol 284:L1012-L1019.

Farne HA, Wilson A, Powell C, Bax L, and Milan SJ (2017) Anti-IL5 therapies for asthma. Cochrane Database Syst Rev 9:CD010834.

Fauler J and Frölich JC (1997) Cigarette smoking stimulates cysteinyl leukotriene production in man. Eur $J$ Clin Invest 27:43-47.
Feoktistov I and Biaggioni I (1995) Adenosine A2b receptors evoke interleukin- 8 secretion in human mast cells: an enprofylline-sensitive mechanism with implications for asthma. $J$ Clin Invest 96:1979-1986.

Feoktistov I, Garland EM, Goldstein AE, Zeng D, Belardinelli L, Wells JN, and Biaggioni I (2001) Inhibition of human mast cell activation with the novel selective adenosine $\mathrm{A}(2 \mathrm{~B})$ receptor antagonist 3-isobutyl-8-pyrrolidinoxanthine (IPDX)(2). Biochem Pharmacol 62:1163-1173.

Ferguson GT, Funck-Brentano C, Fischer T, Darken P, and Reisner C (2003) Cardiovascular safety of salmeterol in COPD. Chest 123:1817-1824.

Ferkol T and Schraufnagel D (2014) The global burden of respiratory disease. Ann Am Thorac Soc 11:404-406.

Fernandes LB, Fryer AD, and Hirshman CA (1992) M2 muscarinic receptors inhibit isoproterenol-induced relaxation of canine airway smooth muscle. $J$ Pharmacol Exp Ther 262:119-126.

Ferrando M, Bagnasco D, Braido F, Baiardini I, Passalacqua G, Puggioni F, Varricch G, and Canonica GW (2017) Umeclidinium for the treatment of uncontrolled asthma. Expert Opin Investig Drugs 26:761-766.

Fish JE, Israel E, Murray JJ, Emmett A, Boone R, Yancey SW, and Rickard KA (2001) Salmeterol powder provides significantly better benefit than montelukast in asthmatic patients receiving concomitant inhaled corticosteroid therapy. Chest 120:423-430.

Fish JE, Kemp JP, Lockey RF, Glass M, Hanby LA, and Bonuccelli CM; The Zafirlukast Trialists Group (1997) Zafirlukast for symptomatic mild-to-moderate asthma: a 13-week multicenter study. Clin Ther 19:675-690.

Foster A and Chan CC (1991) Peptide leukotriene involvement in pulmonary eosinophil migration upon antigen challenge in the actively sensitized guinea pig. Int Arch Allergy Appl Immunol 96:279-284.

Fowler A, Koenen R, Hilbert J, Blatchford J, Kappeler D, Benediktus E, Wood C, and Gupta A (2017) Safety, tolerability, pharmacokinetics, and pharmacodynamics of the novel CRTH2 antagonist BI 1021958 at single oral doses in healthy men and multiple oral doses in men and women with well-controlled asthma. J Clin Pharmacol 57:1444-1453.

Fredriksson R, Lagerström MC, Lundin LG, and Schiöth HB (2003) The G-proteincoupled receptors in the human genome form five main families: phylogenetic analysis, paralogon groups, and fingerprints. Mol Pharmacol 63:1256-1272.

Fregonese L, Silvestri M, Sabatini F, and Rossi GA (2002) Cysteinyl leukotrienes induce human eosinophil locomotion and adhesion molecule expression via a CysLT1 receptor-mediated mechanism. Clin Exp Allergy 32:745-750.

Fretz H, Valdenaire A, Pothier J, Hilpert K, Gnerre C, Peter O, Leroy X, and Riederer MA (2013) Identification of 2-(2-(1-naphthoyl)-8-fluoro-3,4-dihydro-1H-pyrido[4,3b]indol-5(2H)-yl)acetic acid (setipiprant/ACT-129968), a potent, selective, and orally bioavailable chemoattractant receptor-homologous molecule expressed on Th2 cells (CRTH2) antagonist. J Med Chem 56:4899-4911.

Fryer AD and Jacoby DB (1998) Muscarinic receptors and control of airway smooth muscle. Am J Respir Crit Care Med 158:S154-S160.

Fukushima C, Shimoda T, Matsuse H, Matsuo N, Takao A, Obase Y, Kohno S, and Asai S (1998) Effect of synthetase inhibitors and receptor antagonists in antigen-induced contraction of human lung parenchyma. Ann Allergy Asthma Immunol 80:245-250.

Funk CD (2001) Prostaglandins and leukotrienes: advances in eicosanoid biology. Science 294:1871-1875.

Galandrin S and Bouvier M (2006) Distinct signaling profiles of beta1 and beta2 adrenergic receptor ligands toward adenylyl cyclase and mitogen-activated protein kinase reveals the pluridimensionality of efficacy. Mol Pharmacol 70:1575-1584.

Garland SG, Smith SM, and Gums JG (2019) Erenumab: a first-in-class monoclonal antibody for migraine prevention. Ann Pharmacother 53:933-939.

Gauvreau GM, Boulet LP, Cockcroft DW, Baatjes A, Cote J, Deschesnes F, Davis B, Strinich T, Howie K, Duong M, et al. (2008) Antisense therapy against CCR3 and the common beta chain attenuates allergen-induced eosinophilic responses. Am $J$ Respir Crit Care Med 177:952-958.

Gauvreau GM, Pageau R, Séguin R, Carballo D, Gauthier J, D’Anjou H, Campbell H, Watson R, Mistry M, Parry-Billings M, et al. (2011) Dose-response effects of TPI ASM8 in asthmatics after allergen. Allergy 66:1242-1248.

Gavaldà A, Miralpeix M, Ramos I, Otal R, Carreño C, Viñals M, Doménech T, Carcasona C, Reyes B, Vilella D, et al. (2009) Characterization of aclidinium bromide, a novel inhaled muscarinic antagonist, with long duration of action and a favorable pharmacological profile. J Pharmacol Exp Ther 331:740-751.

Gavaldà A, Ramos I, Carcasona C, Calama E, Otal R, Montero JL, Sentellas S, Aparici M, Vilella D, Alberti J, et al. (2014) The in vitro and in vivo profile of aclidinium bromide in comparison with glycopyrronium bromide. Pulm Pharmacol Ther 28:114-121.

Gelfand EW (2017) Importance of the leukotriene B4-BLT1 and LTB4-BLT2 pathways in asthma. Semin Immunol 33:44-51.

Gervais FG, Cruz RP, Chateauneuf A, Gale S, Sawyer N, Nantel F, Metters KM, and O'neill GP (2001) Selective modulation of chemokinesis, degranulation, and apoptosis in eosinophils through the PGD2 receptors CRTH2 and DP. J Allergy Clin Immunol 108:982-988.

Gilman MJ, Meyer L, Carter J, and Slovis C (1990) Comparison of aerosolized glycopyrrolate and metaproterenol in acute asthma. Chest 98:1095-1098.

Gimenez LE, Baameur F, Vayttaden SJ, and Clark RB (2015) Salmeterol efficacy and bias in the activation and kinase-mediated desensitization of $\beta 2$-adrenergic receptors. Mol Pharmacol 87:954-964.

Giouleka P, Papatheodorou G, Lyberopoulos P, Karakatsani A, Alchanatis M, Roussos C, Papiris S, and Loukides S (2011) Body mass index is associated with leukotriene inflammation in asthmatics. Eur J Clin Invest 41:30-38.

Gnagey AL, Seidenberg M, and Ellis J (1999) Site-directed mutagenesis reveals two epitopes involved in the subtype selectivity of the allosteric interactions of gallamine at muscarinic acetylcholine receptors. Mol Pharmacol 56:1245-1253.

Goadsby PJ, Reuter U, Hallström Y, Broessner G, Bonner JH, Zhang F, Sapra S, Picard H, Mikol DD, and Lenz RA (2017) A controlled trial of erenumab for episodic migraine. N Engl J Med 377:2123-2132. 
Goldstein S (2019) Clinical efficacy and safety of anticholinergic therapies in pediatric patients. Ther Clin Risk Manag 15:437-449.

Gomes RF and Bates JH (2002) Geometric determinants of airway resistance in two isomorphic rodent species. Respir Physiol Neurobiol 130:317-325.

Gonem S, Berair R, Singapuri A, Hartley R, Laurencin MFM, Bacher G, Holzhauer B, Bourne M, Mistry V, Pavord ID, et al. (2016) Fevipiprant, a prostaglandin D2 receptor 2 antagonist, in patients with persistent eosinophilic asthma: a singlecentre, randomised, double-blind, parallel-group, placebo-controlled trial. Lancet Respir Med 4:699-707.

Gorenne I, Labat C, Gascard JP, Norel X, Nashashibi N, and Brink C (1998) Leukotriene D4 contractions in human airways are blocked by SK\&F 96365 , an inhibitor of receptor-mediated calcium entry. J Pharmacol Exp Ther 284:549-552.

Gosens R and Gross N (2018) The mode of action of anticholinergics in asthma. Eur Respir J 52:1701247.

Gosens R, Zaagsma J, Meurs H, and Halayko AJ (2006) Muscarinic receptor signaling in the pathophysiology of asthma and COPD. Respir Res 7:73.

Grandordy BM, Cuss FM, Sampson AS, Palmer JB, and Barnes PJ (1986) Phosphatidylinositol response to cholinergic agonists in airway smooth muscle: relationship to contraction and muscarinic receptor occupancy. J Pharmacol Exp Ther 238:273-279.

Grassin-Delyle S, Abrial C, Fayad-Kobeissi S, Brollo M, Faisy C, Alvarez JC, Naline $\mathrm{E}$, and Devillier P (2013) The expression and relaxant effect of bitter taste receptors in human bronchi. Respir Res 14:134.

Green RH, Brightling CE, Woltmann G, Parker D, Wardlaw AJ, and Pavord ID (2002) Analysis of induced sputum in adults with asthma: identification of subgroup with isolated sputum neutrophilia and poor response to inhaled corticosteroids. Thorax 57:875-879.

Gregorio GG, Masureel M, Hilger D, Terry DS, Juette M, Zhao H, Zhou Z, PerezAguilar JM, Hauge M, Mathiasen S, et al. (2017) Single-molecule analysis of ligand efficacy in $\beta_{2}$ AR-G-protein activation. Nature 547:68-73.

Gregory KJ, Sexton PM, and Christopoulos A (2007) Allosteric modulation of muscarinic acetylcholine receptors. Curr Neuropharmacol 5:157-167.

Gross NJ (2006) Anticholinergic agents in asthma and COPD. Eur J Pharmacol 533: $36-39$.

Gross NJ and Barnes PJ (2017) New therapies for asthma and chronic obstructive pulmonary disease. Am J Respir Crit Care Med 195:159-166.

Gross NJ and Skorodin MS (1984) Role of the parasympathetic system in airway obstruction due to emphysema. $N$ Engl J Med 311:421-425.

Grünig G, Warnock M, Wakil AE, Venkayya R, Brombacher F, Rennick DM, Sheppard D, Mohrs M, Donaldson DD, Locksley RM, et al. (1998) Requirement for IL-13 independently of IL-4 in experimental asthma. Science 282:2261-2263.

Gschwandtner M, Mommert S, Köther B, Werfel T, and Gutzmer R (2011) The histamine $\mathrm{H} 4$ receptor is highly expressed on plasmacytoid dendritic cells in psoriasis and histamine regulates their cytokine production and migration. J Invest Dermatol 131:1668-1676.

Gutzmer R, Diestel C, Mommert S, Köther B, Stark H, Wittmann M, and Werfel T (2005) Histamine H4 receptor stimulation suppresses IL-12p70 production and mediates chemotaxis in human monocyte-derived dendritic cells. J Immunol $\mathbf{1 7 4}$ $5224-5232$.

Gyles SL, Xue L, Townsend ER, Wettey F, and Pettipher R (2006) A dominant role for chemoattractant receptor-homologous molecule expressed on T helper type 2 (Th2) cells (CRTH2) in mediating chemotaxis of CRTH2+ CD4+ Th2 lymphocytes in response to mast cell supernatants. Immunology 119:362-368.

Haddad EB, Landry Y, and Gies JP (1991) Muscarinic receptor subtypes in guinea pig airways. Am J Physiol 261:L327-L333.

Haddad EB, Mak JC, Hislop A, Haworth SG, and Barnes PJ (1994) Characterization of muscarinic receptor subtypes in pig airways: radioligand binding and Northern blotting studies. Am J Physiol 266:L642-L648.

Haddad EB, Patel H, Keeling JE, Yacoub MH, Barnes PJ, and Belvisi MG (1999) Pharmacological characterization of the muscarinic receptor antagonist, glycopyrrolate, in human and guinea-pig airways. Br J Pharmacol 127:413-420.

Haga K, Kruse AC, Asada H, Yurugi-Kobayashi T, Shiroishi M, Zhang C, Weis WI, Okada T, Kobilka BK, Haga T, et al. (2012) Structure of the human M2 muscarinic acetylcholine receptor bound to an antagonist. Nature 482:547-551.

Haldar P, Pavord ID, Shaw DE, Berry MA, Thomas M, Brightling CE, Wardlaw AJ, and Green RH (2008) Cluster analysis and clinical asthma phenotypes. Am $J$ Respir Crit Care Med 178:218-224.

Halim TY, Steer CA, Mathä L, Gold MJ, Martinez-Gonzalez I, McNagny KM, McKenzie AN, and Takei F (2014) Group 2 innate lymphoid cells are critical for the initiation of adaptive $\mathrm{T}$ helper 2 cell-mediated allergic lung inflammation. Immunity 40:425-435.

Hall IP, Fowler AV, Gupta A, Tetzlaff K, Nivens MC, Sarno M, Finnigan HA, Bateman ED, and Rand Sutherland E (2015) Efficacy of BI 671800, an oral CRTH2 antagonist, in poorly controlled asthma as sole controller and in the presence of inhaled corticosteroid treatment. Pulm Pharmacol Ther 32:37-44.

Hamilton A, Faiferman I, Stober P, Watson RM, and O'Byrne PM (1998) Pranlukast, a cysteinyl leukotriene receptor antagonist, attenuates allergen-induced early- and late-phase bronchoconstriction and airway hyperresponsiveness in asthmatic subjects. J Allergy Clin Immunol 102:177-183.

Hammad H, Chieppa M, Perros F, Willart MA, Germain RN, and Lambrecht BN (2009) House dust mite allergen induces asthma via Toll-like receptor 4 triggering of airway structural cells. Nat Med 15:410-416

Hammad H, de Heer HJ, Soullie T, Hoogsteden HC, Trottein F, and Lambrecht BN (2003) Prostaglandin D2 inhibits airway dendritic cell migration and function in steady state conditions by selective activation of the D prostanoid receptor 1 . J Immunol 171:3936-3940.

Hammer R, Berrie CP, Birdsall NJ, Burgen AS, and Hulme EC (1980) Pirenzepine distinguishes between different subclasses of muscarinic receptors. Nature 283:90-92

Hammer R and Giachetti A (1982) Muscarinic receptor subtypes: M1 and M2 biochemical and functional characterization. Life Sci 31:2991-2998.
Hanania NA, Mannava B, Franklin AE, Lipworth BJ, Williamson PA, Garner WJ, Dickey BF, and Bond RA (2010) Response to salbutamol in patients with mild asthma treated with nadolol. Eur Respir J 36:963-965.

Hanania NA, Singh S, El-Wali R, Flashner M, Franklin AE, Garner WJ, Dickey BF, Parra S, Ruoss S, Shardonofsky F, et al. (2008) The safety and effects of the betablocker, nadolol, in mild asthma: an open-label pilot study. Pulm Pharmacol Ther 21:134-141.

Hanoune J and Defer N (2001) Regulation and role of adenylyl cyclase isoforms. Annu Rev Pharmacol Toxicol 41:145-174.

Hanson MA, Cherezov V, Griffith MT, Roth CB, Jaakola VP, Chien EY, Velasquez J, Kuhn P, and Stevens RC (2008) A specific cholesterol binding site is established by the 2.8 A structure of the human beta2-adrenergic receptor. Structure 16:897-905. Harmsen MM and De Haard HJ (2007) Properties, production, and applications of camelid single-domain antibody fragments. Appl Microbiol Biotechnol 77:13-22.

Harnett KM and Biancani P (2003) Calcium-dependent and calcium-independent contractions in smooth muscles. Am J Med 115 (Suppl 3A):24S-30S.

Hata AN and Breyer RM (2004) Pharmacology and signaling of prostaglandin receptors: multiple roles in inflammation and immune modulation. Pharmacol Ther 103:147-166.

Hauser AS, Attwood MM, Rask-Andersen M, Schiöth HB, and Gloriam DE (2017) Trends in GPCR drug discovery: new agents, targets and indications. Nat Rev Drug Discov 16:829-842.

Hawlader MD, Ma E, Noguchi E, Itoh M, Arifeen SE, Persson LA, Moore SE, Raqib R, and Wagatsuma Y (2014) Ascaris lumbricoids infection as a risk factor for asthma and atopy in rural Bangladeshi children. Trop Med Health 42:77-85.

He HQ and Ye RD (2017) The formyl peptide receptors: diversity of ligands and mechanism for recognition. Molecules 22:E455.

Heinemann A, Schuligoi R, Sabroe I, Hartnell A, and Peskar BA (2003) Delta 12 prostaglandin J2, a plasma metabolite of prostaglandin D2, causes eosinophil mobilization from the bone marrow and primes eosinophils for chemotaxis. $J$ Immunol 170:4752-4758.

Henderson WR Jr, Lewis DB, Albert RK, Zhang Y, Lamm WJ, Chiang GK, Jones F, Eriksen P, Tien YT, Jonas M, et al. (1996) The importance of leukotrienes in airway inflammation in a mouse model of asthma. J Exp Med 184:1483-1494.

Henderson WR Jr, Tang LO, Chu SJ, Tsao SM, Chiang GK, Jones F, Jonas M, Pae C, Wang H, and Chi EY (2002) A role for cysteinyl leukotrienes in airway remodeling in a mouse asthma model. Am J Respir Crit Care Med 165:108-116.

Herszberg B, Ramos-Barbón D, Tamaoka M, Martin JG, and Lavoie JP (2006) Heaves, an asthma-like equine disease, involves airway smooth muscle remodeling. J Allergy Clin Immunol 118:382-388.

Heukers R, Fan TS, de Wit RH, van Senten JR, De Groof TWM, Bebelman MP, Lagerweij T, Vieira J, de Munnik SM, Smits-de Vries L, et al. (2018) The constitutive activity of the virally encoded chemokine receptor US28 accelerates glioblastoma growth. Oncogene 37:4110-4121.

Hicks A, Goodnow R Jr, Cavallo G, Tannu SA, Ventre JD, Lavelle D, Lora JM, Satjawatcharaphong J, Brovarney M, Dabbagh K, et al. (2010) Effects of LTB4 receptor antagonism on pulmonary inflammation in rodents and non-human primates. Prostaglandins Other Lipid Mediat 92:33-43.

Hicks A, Monkarsh SP, Hoffman AF, and Goodnow R Jr (2007) Leukotriene B4 receptor antagonists as therapeutics for inflammatory disease: preclinical and clinical developments. Expert Opin Investig Drugs 16:1909-1920.

Hilger D, Masureel M, and Kobilka BK (2018) Structure and dynamics of GPCR signaling complexes. Nat Struct Mol Biol 25:4-12.

Hirai H, Tanaka K, Yoshie O, Ogawa K, Kenmotsu K, Takamori Y, Ichimasa M, Sugamura K, Nakamura M, Takano S, et al. (2001) Prostaglandin D2 selectively induces chemotaxis in T helper type 2 cells, eosinophils, and basophils via seventransmembrane receptor CRTH2. J Exp Med 193:255-261.

Hofstra CL, Desai PJ, Thurmond RL, and Fung-Leung WP (2003) Histamine H4 receptor mediates chemotaxis and calcium mobilization of mast cells. J Pharmacol Exp Ther 305:1212-1221.

Hogg JC (2004) Pathophysiology of airflow limitation in chronic obstructive pulmonary disease. Lancet 364:709-721.

Holgate ST (2005) The Quintiles Prize Lecture 2004: the identification of the adenosine A2B receptor as a novel therapeutic target in asthma. Br J Pharmacol 145 $1009-1015$

Hollingsworth JW, Theriot BS, Li Z, Lawson BL, Sunday M, Schwartz DA and Walker JK (2010) Both hematopoietic-derived and non-hematopoietic-derived beta-arrestin-2 regulates murine allergic airway disease. Am J Respir Cell Mol Biol 43:269-275.

Holroyde MC, Altounyan RE, Cole M, Dixon M, and Elliott EV (1981) Bronchoconstriction produced in man by leukotrienes C and D. Lancet 2:17-18.

Horiguchi T, Tachikawa S, Kasahara J, Doi M, and Shiga M (1999) Effects of pranlukast hydrate on serum eosinophil cationic protein levels in patients with adult bronchial asthma. Arzneimittelforschung 49:35-37.

Horiguchi T, Tachikawa S, Kondo R, Miyazaki J, Shiga M, Hirose M, Kobayashi K, Hayashi N, Ohira D, Nasu T, et al. (2007) Comparative evaluation of the leukotriene receptor antagonist pranlukast versus the steroid inhalant fluticasone in the therapy of aged patients with mild bronchial asthma. Arzneimittelforschung 57: 87-91.

Hoshino M, Izumi T, and Shimizu T (1998) Leukotriene D4 activates mitogenactivated protein kinase through a protein kinase Calpha-Raf-1-dependent pathway in human monocytic leukemia THP-1 cells. J Biol Chem 273:4878-4882.

Hosokawa H, Tanaka T, Kato M, Shinoda K, Tohyama H, Hanazawa A, Tamaki Y, Hirahara K, Yagi R, Sakikawa I, et al. (2013) Gata3/Ruvbl2 complex regulates T helper 2 cell proliferation via repression of Cdkn2c expression. Proc Natl Acad Sci USA 110:18626-18631.

House SL, Matsuda K, O’Brien G, Makhay M, Iwaki Y, Ferguson I, Lovato LM, and Lewis LM (2015) Efficacy of a new intravenous $\beta 2$-adrenergic agonist (bedoradrine, MN-221) for patients with an acute exacerbation of asthma. Respir Med 109:1268-1273. 
Huang CY, Olieric V, Ma P, Howe N, Vogeley L, Liu X, Warshamanage R, Weinert T, Panepucci E, Kobilka B, et al. (2016a) In meso in situ serial X-ray crystallography of soluble and membrane proteins at cryogenic temperatures. Acta Crystallogr D Struct Biol 72:93-112.

Huang T, Hazen M, Shang Y, Zhou M, Wu X, Yan D, Lin Z, Solon M, Luis E, Ngu H, et al. (2016b) Depletion of major pathogenic cells in asthma by targeting CRTh2. JCI Insight 1:e86689.

Huang X, Brubaker J, Zhou W, Biju PJ, Xiao L, Shao N, Huang Y, Dong L, Liu Z, Bitar R, et al. (2018) Discovery of MK-8318, a potent and selective CRTh2 receptor antagonist for the treatment of asthma. ACS Med Chem Lett 9:679-684.

Hui Y and Funk CD (2002) Cysteinyl leukotriene receptors. Biochem Pharmacol 64: 1549-1557.

Huszár E, Vass G, Vizi E, Csoma Z, Barát E, Molnár Világos G, Herjavecz I, and Horváth I (2002) Adenosine in exhaled breath condensate in healthy volunteers and in patients with asthma. Eur Respir $J$ 20:1393-1398.

Hutchings CJ, Koglin M, Olson WC, and Marshall FH (2017) Opportunities for therapeutic antibodies directed at G-protein-coupled receptors. Nat Rev Drug Discov 16:787-810.

Ikeda G, Miyahara N, Koga H, Fuchimoto Y, Waseda K, Kurimoto E, Taniguchi A, Tanimoto Y, Kataoka M, Tanimoto M, et al. (2014) Effect of a cysteinyl leukotriene receptor antagonist on experimental emphysema and asthma combined with emphysema. Am J Respir Cell Mol Biol 50:18-29.

Imaoka H, Campbell H, Babirad I, Watson RM, Mistry M, Sehmi R, and Gauvreau GM (2011) TPI ASM8 reduces eosinophil progenitors in sputum after allergen challenge. Clin Exp Allergy 41:1740-1746.

Inman WH and Adelstein AM (1969) Rise and fall of asthma mortality in England and Wales in relation to use of pressurised aerosols. Lancet 2:279-285.

Inoue Y, Yoshizato T, and Kawarabayashi T (2009) Investigation of beta(2)-adrenoceptor subtype selectivity and organ specificity for bedoradrine (KUR-1246), a novel tocolytic beta-adrenergic receptor stimulant. J Obstet Gynaecol Res 35:405-413.

Insel PA, Sriram K, Wiley SZ, Wilderman A, Katakia T, McCann T, Yokouchi H, Zhang L, Corriden R, Liu D, et al. (2018) GPCRomics: GPCR expression in cancer cells and tumors identifies new, potential biomarkers and therapeutic targets. Front Pharmacol 9:431.

Ishii M and Kurachi Y (2006) Muscarinic acetylcholine receptors. Curr Pharm Des 12: 3573-3581.

Ishioka S, Hozawa S, Haruta Y, Hiyama K, Maeda A, and Yamakido M (1999) Pranlukast, a cysteinyl leukotriene antagonist, reduces serum eosinophil cationic protein levels in patients with asthma. Hiroshima J Med Sci 48:105-110.

Ishioka S, Hozawa S, Haruta Y, Maeda A, Tamagawa K, Watanabe T, Hiyama K, and Yamakido M (2000) Effects of pranlukast, a cysteinyl leukotriene antagonist, on bronchial responsiveness to methacholine in aspirin-intolerant asthmatics treated with corticosteroids. Hiroshima J Med Sci 49:105-108.

Ishizuka T, Matsui T, Okamoto Y, Ohta A, and Shichijo M (2004) Ramatroban (BAY $\mathrm{u}$ 3405): a novel dual antagonist of TXA2 receptor and CRTh2, a newly identified prostaglandin D2 receptor. Cardiovasc Drug Rev 22:71-90.

Isogaya M, Yamagiwa Y, Fujita S, Sugimoto Y, Nagao T, and Kurose H (1998) Identification of a key amino acid of the beta2-adrenergic receptor for high affinity binding of salmeterol. Mol Pharmacol 54:616-622.

Jackson M (2010) "Divine stramonium": the rise and fall of smoking for asthma. Med Hist 54:171-194.

Jähnichen S, Blanchetot C, Maussang D, Gonzalez-Pajuelo M, Chow KY, Bosch L, De Vrieze S, Serruys B, Ulrichts H, Vandevelde W, et al. (2010) CXCR4 nanobodies (VHH-based single variable domains) potently inhibit chemotaxis and HIV-1 replication and mobilize stem cells. Proc Natl Acad Sci USA 107:20565-20570.

Jakubík J, Krejcí A, and Dolezal V (2005) Asparagine, valine, and threonine in the third extracellular loop of muscarinic receptor have essential roles in the positive cooperativity of strychnine-like allosteric modulators. J Pharmacol Exp Ther 313: 688-696.

January B, Seibold A, Allal C, Whaley BS, Knoll BJ, Moore RH, Dickey BF, Barber $\mathrm{R}$, and Clark RB (1998) Salmeterol-induced desensitization, internalization and phosphorylation of the human beta2-adrenoceptor. Br J Pharmacol 123:701-711.

January B, Seibold A, Whaley B, Hipkin RW, Lin D, Schonbrunn A, Barber R, and Clark RB (1997) Beta2-adrenergic receptor desensitization, internalization, and phosphorylation in response to full and partial agonists. J Biol Chem 272:23871-23879.

Jatakanon A, Uasuf C, Maziak W, Lim S, Chung KF, and Barnes PJ (1999) Neutrophilic inflammation in severe persistent asthma. Am J Respir Crit Care Med 160:1532-1539.

Jeffery PK (2001) Remodeling in asthma and chronic obstructive lung disease. Am $J$ Respir Crit Care Med 164:S28-S38.

Jemima EA, Prema A, and Thangam EB (2014) Functional characterization of histamine H4 receptor on human mast cells. Mol Immunol 62:19-28.

Jiang Y, Borrelli LA, Kanaoka Y, Bacskai BJ, and Boyce JA (2007) CysLT2 receptors interact with CysLT1 receptors and down-modulate cysteinyl leukotriene dependent mitogenic responses of mast cells. Blood 110:3263-3270

Jiang Y, Kanaoka Y, Feng C, Nocka K, Rao S, and Boyce JA (2006) Cutting edge: interleukin 4-dependent mast cell proliferation requires autocrine/intracrine cysteinyl leukotriene-induced signaling. J Immunol 177:2755-2759.

Johnson HG, Chinn RA, Chow AW, Bach MK, and Nadel JA (1983) Leukotriene-C4 enhances mucus production from submucosal glands in canine trachea in vivo. Int J Immunopharmacol 5:391-396.

Jones SV, Heilman CJ, and Brann MR (1991) Functional responses of cloned muscarinic receptors expressed in CHO-K1 cells. Mol Pharmacol 40:242-247.

Jones TR, Labelle M, Belley M, Champion E, Charette L, Evans J, Ford-Hutchinson AW, Gauthier JY, Lord A, Masson P, et al. (1995) Pharmacology of montelukast sodium (Singulair), a potent and selective leukotriene D4 receptor antagonist. Can J Physiol Pharmacol 73:191-201.

Joos S, Miksch A, Szecsenyi J, Wieseler B, Grouven U, Kaiser T, and Schneider A (2008) Montelukast as add-on therapy to inhaled corticosteroids in the treatment of mild to moderate asthma: a systematic review. Thorax 63:453-462.
Joost A and Kolkman DAL (2010) Nanobodies - from llamas to therapeutic proteins. Drug Discov Today Technol 7:e95-e146.

Joris I, Majno G, Corey EJ, and Lewis RA (1987) The mechanism of vascular leakage induced by leukotriene E4: endothelial contraction. Am J Pathol 126:19-24.

Joshi R, Valdez D, Kim H, Eikenburg DC, Knoll BJ, and Bond RA (2017) Effects of $\beta$-blockers on house dust mite-driven murine models pre- and post-development of an asthma phenotype. Pulm Pharmacol Ther 46:30-40.

Juan A and Ballesteros HW (1995) Integrated methods for the construction of threedimensional models and computational probing of structure-function relations in $\mathrm{G}$ protein-coupled receptors. Methods Neurosci 25:366-428.

Kabata H, Moro K, Fukunaga K, Suzuki Y, Miyata J, Masaki K, Betsuyaku T, Koyasu S, and Asano K (2013) Thymic stromal lymphopoietin induces corticosteroid resistance in natural helper cells during airway inflammation. Nat Commun 4: 2675 .

Kalla RV and Zablocki J (2009) Progress in the discovery of selective, high affinity $\mathrm{A}(2 \mathrm{~B})$ adenosine receptor antagonists as clinical candidates. Purinergic Signal 5: $21-29$

Kamal M and Jockers R (2009) Bitopic ligands: all-in-one orthosteric and allosteric. F1000 Biol Rep 1:77.

Kanaoka Y and Boyce JA (2014) Cysteinyl leukotrienes and their receptors; emerging concepts. Allergy Asthma Immunol Res 6:288-295.

Kanaoka Y, Maekawa A, and Austen KF (2013) Identification of GPR99 protein as a potential third cysteinyl leukotriene receptor with a preference for leukotriene E4 ligand. J Biol Chem 288:10967-10972.

Kang JY, Rhee CK, Kim JS, Park CK, Kim SJ, Lee SH, Yoon HK, Kwon SS, Kim YK, and Lee SY (2012) Effect of tiotropium bromide on airway remodeling in a chronic asthma model. Ann Allergy Asthma Immunol 109:29-35.

Kanwar S, Johnston B, and Kubes P (1995) Leukotriene C4/D4 induces P-selectin and sialyl Lewis(x)-dependent alterations in leukocyte kinetics in vivo. Circ Res 77. 879-887.

Kaplon H and Reichert JM (2018) Antibodies to watch in 2018. MAbs 10:183-203. Kaplon H and Reichert JM (2019) Antibodies to watch in 2019. MAbs 11:219-238.

Kasprowicz DJ, Kohm AP, Berton MT, Chruscinski AJ, Sharpe A, and Sanders VM (2000) Stimulation of the B cell receptor, CD86 (B7-2), and the beta 2-adrenergic receptor intrinsically modulates the level of IgG1 and IgE produced per B cell. J Immunol 165:680-690.

Kaur M, Holden N, Wilson S, Sukkar M, Chung KF, Barnes P, Newton R, Giembycz $M$, et al. (2008) Effect of $\beta_{2}$-adrenoceptor agonists and other cAMP-elevating agents on inflammatory gene expression in human ASM cells: a role for protein kinase A. American Journal of Physiology-Lung Cellular and Molecular Physiology Sep; 295(3):L505-14. 18586957.

Kaur M, Chivers J, Giembycz M, Newton R, et al. (2008) Long-Acting $\beta_{2}$-Adrenoceptor Agonists Synergistically Enhance Glucocorticoid-Dependent Transcription in Human Airway Epithelial and Smooth Muscle Cells. Molecular Pharmacology Jan;73(1):203-14. 17901197.

Kazani S and Israel E (2013) What doesn't kill may not make you stronger: $\beta$-blockers for asthma. Am J Respir Crit Care Med 187:1281.

Kazani S and Israel E (2014) Reply: "The intrinsic bias of generalizations" and "Far from 'disappointing". Am J Respir Crit Care Med 189:361-362.

Keam SJ, Lyseng-Williamson KA, and Goa KL (2003) Pranlukast: a review of its use in the management of asthma. Drugs 63:991-1019.

Kemp JP, Dockhorn RJ, Shapiro GG, Nguyen HH, Reiss TF, Seidenberg BC, and Knorr B (1998) Montelukast once daily inhibits exercise-induced bronchoconstriction in 6- to 14-year-old children with asthma. J Pediatr 133:424-428.

Kemp JP, Minkwitz MC, Bonuccelli CM, and Warren MS (1999) Therapeutic effect of zafirlukast as monotherapy in steroid-naive patients with severe persistent asthma. Chest 115:336-342.

Keov P, Sexton PM, and Christopoulos A (2011) Allosteric modulation of G proteincoupled receptors: a pharmacological perspective. Neuropharmacology 60:24-35.

Kerstjens HA, Moroni-Zentgraf P, Tashkin DP, Dahl R, Paggiaro P, Vandewalker M, Schmidt H, Engel M, and Bateman ED (2016) Tiotropium improves lung function, exacerbation rate, and asthma control, independent of baseline characteristics including age, degree of airway obstruction, and allergic status. Respir Med 117: 198-206.

Kiguchi S, Matsuda T, Cho K, Okuyama K, Akahane M, and Fujimoto S (2002) KUR1246, a novel beta(2)-adrenoceptor agonist, as a tocolytic agent. Obstet Gynecol 100: 487-494

Kim BS, Wojno ED, and Artis D (2013) Innate lymphoid cells and allergic inflammation. Curr Opin Immunol 25:738-744.

Kim HY, Lee HJ, Chang YJ, Pichavant M, Shore SA, Fitzgerald KA, Iwakura Y, Israel E, Bolger K, Faul J, et al. (2014) Interleukin-17-producing innate lymphoid cells and the NLRP3 inflammasome facilitate obesity-associated airway hyperreactivity. Nat Med 20:54-61.

Kim YH, Bagot M, Pinter-Brown L, Rook AH, Porcu P, Horwitz SM, Whittaker S, Tokura Y, Vermeer M, Zinzani PL, et al.; MAVORIC Investigators (2018) Mogamulizumab versus vorinostat in previously treated cutaneous T-cell lymphoma (MAVORIC): an international, open-label, randomised, controlled phase 3 trial. Lancet Oncol 19:1192-1204.

Kistemaker LE, Bos IS, Menzen MH, Maarsingh H, Meurs H, and Gosens R (2016) Combination therapy of tiotropium and ciclesonide attenuates airway inflammation and remodeling in a guinea pig model of chronic asthma. Respir Res $\mathbf{1 7}$ 13.

Kistemaker LE, Bos ST, Mudde WM, Hylkema MN, Hiemstra PS, Wess J, Meurs H, Kerstjens HA, and Gosens R (2014) Muscarinic $\mathrm{M}_{3}$ receptors contribute to allergeninduced airway remodeling in mice. Am J Respir Cell Mol Biol 50:690-698.

Kistemaker LE and Gosens R (2015) Acetylcholine beyond bronchoconstriction: roles in inflammation and remodeling. Trends Pharmacol Sci 36:164-171.

Kistemaker LE, Oenema TA, Meurs H, and Gosens R (2012) Regulation of airway inflammation and remodeling by muscarinic receptors: perspectives on anticholinergic therapy in asthma and COPD. Life Sci 91:1126-1133. 
Klarenbeek A, Maussang D, Blanchetot C, Saunders M, van der Woning S, Smit M, de Haard H, and Hofman E (2012) Targeting chemokines and chemokine receptors with antibodies. Drug Discov Today Technol 9:e227-e314.

Knight JM, Mak G, Shaw J, Porter P, McDermott C, Roberts L, You R, Yuan X, Millien VO, Qian Y, et al. (2015) Long-acting beta agonists enhance allergic airway disease. PLoS One 10:e0142212.

Kobayashi M, Takeda K, Murata S, Kojima M, Akahane M, Inoue Y, Kitamura K, and Kawarabayashi T (2001) Pharmacological characterization of KUR-1246, a selective uterine relaxant. J Pharmacol Exp Ther 297:666-671.

Kohrogi H, Iwagoe H, Fujii K, Hamamoto J, Fukuda K, Hirata N, Kawano O, Matsumoto M, Suga M, and Ando M (1999) The role of cysteinyl leukotrienes in the pathogenesis of asthma: clinical study of leukotriene antagonist pranlukast for 1 year in moderate and severe asthma. Respirology 4:319-323.

Kollmeier AP, Barnathan ES, O’Brien C, Chen B, Xia YK, Zhou B, Loza MJ, Silkoff $\mathrm{PE}, \mathrm{Ge} \mathrm{M}$, and Thurmond RL (2018) A phase $2 \mathrm{a}$ study of toreforant, a histamine H4 receptor antagonist, in eosinophilic asthma. Ann Allergy Asthma Immunol 121: $568-574$.

Korczynska M, Clark MJ, Valant C, Xu J, Moo EV, Albold S, Weiss DR, Torosyan H, Huang W, Kruse AC, et al. (2018) Structure-based discovery of selective positive allosteric modulators of antagonists for the $\mathrm{M}_{2}$ muscarinic acetylcholine receptor. Proc Natl Acad Sci USA 115:E2419-E2428.

Kostenis E and Ulven T (2006) Emerging roles of DP and CRTH2 in allergic inflammation. Trends Mol Med 12:148-158.

Krejcí A and Tucek S (2001) Changes of cooperativity between N-methylscopolamine and allosteric modulators alcuronium and gallamine induced by mutations of external loops of muscarinic M(3) receptors. Mol Pharmacol 60:761-767.

Krell RD, Aharony D, Buckner CK, Keith RA, Kusner EJ, Snyder DW, Bernstein PR, Matassa VG, Yee YK, Brown FJ, et al. (1990) The preclinical pharmacology of ICI 204,219: a peptide leukotriene antagonist. Am Rev Respir Dis 141:978-987.

Krell RD, Dehaas CJ, Lengel DJ, Kusner EJ, Williams JC, and Buckner CK (1994) Preclinical exploration of the potential antiinflammatory properties of the peptide leukotriene antagonist ICI 204,219 (Accolate). Ann N Y Acad Sci 744:289-298.

Kruse AC, Hu J, Kobilka BK, and Wess J (2014a) Muscarinic acetylcholine receptor X-ray structures: potential implications for drug development. Curr Opin Pharmacol 16:24-30.

Kruse AC, Hu J, Pan AC, Arlow DH, Rosenbaum DM, Rosemond E, Green HF, Liu T, Chae PS, Dror RO, et al. (2012) Structure and dynamics of the M3 muscarinic acetylcholine receptor. Nature 482:552-556.

Kruse AC, Kobilka BK, Gautam D, Sexton PM, Christopoulos A, and Wess J (2014b) Muscarinic acetylcholine receptors: novel opportunities for drug development. Nat Rev Drug Discov 13:549-560.

Kruse AC, Ring AM, Manglik A, Hu J, Hu K, Eitel K, Hübner H, Pardon E, Valant C, Sexton PM, et al. (2013a) Activation and allosteric modulation of a muscarinic acetylcholine receptor. Nature 504:101-106.

Kruse AC, Weiss DR, Rossi M, Hu J, Hu K, Eitel K, Gmeiner P, Wess J, Kobilka BK, and Shoichet BK (2013b) Muscarinic receptors as model targets and antitargets for structure-based ligand discovery. Mol Pharmacol 84:528-540.

Kucharewicz I, Bodzenta-Łukaszyk A, and Buczko W (2008) Experimental asthma in rats. Pharmacol Rep 60:783-788.

Kume H and Kotlikoff MI (1991) Muscarinic inhibition of single KCa channels in smooth muscle cells by a pertussis-sensitive G protein. Am J Physiol 261 C1204-C1209.

Kuna P, Bjermer L, and Tornling G (2016) Two phase II randomized trials on the CRTh2 antagonist AZD1981 in adults with asthma. Drug Des Devel Ther 10: $2759-2770$.

Kuo CS, Pavlidis S, Loza M, Baribaud F, Rowe A, Pandis I, Hoda U, Rossios C, Sousa A, Wilson SJ, et al.; U-BIOPRED Project Team $\leftleftarrows$ (2017) A transcriptome-driven analysis of epithelial brushings and bronchial biopsies to define asthma phenotypes in U-BIOPRED. Am J Respir Crit Care Med 195:443-455.

Kupczyk M and Kuna P (2017) Targeting the $\mathrm{PGD}_{2} / \mathrm{CRTH} 2 / \mathrm{DP} 1$ signaling pathway in asthma and allergic disease: current status and future perspectives. Drugs $\mathbf{7 7}$ $1281-1294$

Kuperman D, Schofield B, Wills-Karp M, and Grusby MJ (1998) Signal transducer and activator of transcription factor 6 (Stat6)-deficient mice are protected from antigen-induced airway hyperresponsiveness and mucus production. $J$ Exp Med 187:939-948

Kurosawa M, Yodonawa S, Tsukagoshi H, and Miyachi Y (1994) Inhibition by a novel peptide leukotriene receptor antagonist ONO-1078 of airway wall thickening and airway hyperresponsiveness to histamine induced by leukotriene $\mathrm{C} 4$ or leukotriene D4 in guinea-pigs. Clin Exp Allergy 24:960-968.

Labat C, Ortiz JL, Norel X, Gorenne I, Verley J, Abram TS, Cuthbert NJ, Tudhope SR, Norman P, Gardiner P, et al. (1992) A second cysteinyl leukotriene receptor in human lung. J Pharmacol Exp Ther 263:800-805.

Laidlaw TM and Boyce JA (2012) Cysteinyl leukotriene receptors, old and new; implications for asthma. Clin Exp Allergy 42:1313-1320.

Laitinen LA, Laitinen A, Haahtela T, Vilkka V, Spur BW, and Lee TH (1993) Leukotriene E4 and granulocytic infiltration into asthmatic airways. Lancet 341:989-990.

Lambrecht BN and Hammad H (2015) The immunology of asthma. Nat Immunol 16 $45-56$

Lambrecht BN, Salomon B, Klatzmann D, and Pauwels RA (1998) Dendritic cells are required for the development of chronic eosinophilic airway inflammation in response to inhaled antigen in sensitized mice. J Immunol 160:4090-4097.

Lane JR, Sexton PM, and Christopoulos A (2013) Bridging the gap: bitopic ligands of G-protein-coupled receptors. Trends Pharmacol Sci 34:59-66.

Langmead CJ, Andrews SP, Congreve M, Errey JC, Hurrell E, Marshall FH, Mason JS, Richardson CM, Robertson N, Zhukov A, et al. (2012) Identification of nove adenosine $\mathrm{A}(2 \mathrm{~A})$ receptor antagonists by virtual screening. $J$ Med Chem $\mathbf{5 5}$ 1904-1909.

Larsson K, Larsson BM, Sandström T, Sundblad BM, and Palmberg L (2001) Sodium cromoglycate attenuates pulmonary inflammation without influencing bronchial responsiveness in healthy subjects exposed to organic dust. Clin Exp Allergy 31: $1356-1368$

Latorraca NR, Venkatakrishnan AJ, and Dror RO (2017) GPCR dynamics: structures in motion. Chem Rev 117:139-155.

Laviolette M, Malmstrom K, Lu S, Chervinsky P, Pujet JC, Peszek I, Zhang J, and Reiss TF; Montelukast/Beclomethasone Additivity Group (1999) Montelukast added to inhaled beclomethasone in treatment of asthma. Am J Respir Crit Care Med 160:1862-1868.

Lazarus SC, Chinchilli VM, Rollings NJ, Boushey HA, Cherniack R, Craig TJ, Deykin A, DiMango E, Fish JE, Ford JG, et al.; National Heart Lung and Blood Institute's Asthma Clinical Research Network (2007) Smoking affects response to inhaled corticosteroids or leukotriene receptor antagonists in asthma. Am J Respir Crit Care Med 175:783-790.

Lee E, Robertson T, Smith J, and Kilfeather S (2000) Leukotriene receptor antagonists and synthesis inhibitors reverse survival in eosinophils of asthmatic individuals. Am J Respir Crit Care Med 161:1881-1886.

Lee LA, Yang S, Kerwin E, Trivedi R, Edwards LD, and Pascoe S (2015) The effect of fluticasone furoate/umeclidinium in adult patients with asthma: a randomized, dose-ranging study. Respir Med 109:54-62.

Lee TA, Pickard AS, Au DH, Bartle B, and Weiss KB (2008) Risk for death associated with medications for recently diagnosed chronic obstructive pulmonary disease. Ann Intern Med 149:380-390.

Leff JA, Busse WW, Pearlman D, Bronsky EA, Kemp J, Hendeles L, Dockhorn R, Kundu S, Zhang J, Seidenberg BC, et al. (1998) Montelukast, a leukotrienereceptor antagonist, for the treatment of mild asthma and exercise-induced bronchoconstriction. $N$ Engl J Med 339:147-152

Lefkowitz RJ (2004) Historical review: a brief history and personal retrospective of seven-transmembrane receptors. Trends Pharmacol Sci 25:413-422.

Lei Y, Gregory JA, Nilsson GP, and Adner M (2013) Insights into mast cell functions in asthma using mouse models. Pulm Pharmacol Ther 26:532-539.

Leppik RA, Miller RC, Eck M, and Paquet JL (1994) Role of acidic amino acids in the allosteric modulation by gallamine of antagonist binding at the $\mathrm{m} 2$ muscarinic acetylcholine receptor. Mol Pharmacol 45:983-990.

Lew W, Chen X, and Kim CU (2000) Discovery and development of GS 4104 (oseltamivir): an orally active influenza neuraminidase inhibitor. Curr Med Chem 7 663-672.

Lewis RA, Austen KF, Drazen JM, Clark DA, Marfat A, and Corey EJ (1980) Slow reacting substances of anaphylaxis: identification of leukotrienes C-1 and D from human and rat sources. Proc Natl Acad Sci USA 77:3710-3714.

Licari A, Castagnoli R, Brambilla I, Marseglia A, Tosca MA, Marseglia GL, and Ciprandi G (2018) Asthma endotyping and biomarkers in childhood asthma. Pediatr Allergy Immunol Pulmonol 31:44-55.

Lim VJY, Du W, Chen YZ, and Fan H (2018) A benchmarking study on virtual ligand screening against homology models of human GPCRs. Proteins 86:978-989.

Lipworth B, Short P, Anderson W, and Williamson P (2014) Reply: pharmacological obfuscation of clinical relevance. Am J Respir Crit Care Med 189:360-361.

Lipworth BJ, Anderson WJ, and Short PM (2016) From mouse to man: predicting biased effects of beta-blockers in asthma. $\mathrm{Br} J$ Pharmacol 173:248-249.

Littmann T, Göttle M, Reinartz MT, Kälble S, Wainer IW, Ozawa T, and Seifert R (2015) Recruitment of $\beta$-arrestin 1 and 2 to the $\beta 2$-adrenoceptor: analysis of 65 ligands. J Pharmacol Exp Ther 355:183-190.

Liu H, Hofmann J, Fish I, Schaake B, Eitel K, Bartuschat A, Kaindl J, Rampp H, Banerjee A, Hübner H, et al. (2018) Structure-guided development of selective M3 muscarinic acetylcholine receptor antagonists. Proc Natl Acad Sci USA 115: 12046-12050.

Liu J, Li AR, Wang Y, Johnson MG, Su Y, Shen W, Wang X, Lively S, Brown M, Lai S, et al. (2011) Discovery of AMG 853, a CRTH2 and DP dual antagonist. ACS Med Chem Lett 2:326-330.

Liu JJ, Horst R, Katritch V, Stevens RC, and Wüthrich K (2012) Biased signaling pathways in $\beta 2$-adrenergic receptor characterized by 19F-NMR. Science 335: $1106-1110$

Liu M and Yokomizo T (2015) The role of leukotrienes in allergic diseases. Allergol Int 64:17-26.

Liu X, Ahn S, Kahsai AW, Meng KC, Latorraca NR, Pani B, Venkatakrishnan AJ, Masoudi A, Weis WI, Dror RO, et al. (2017) Mechanism of intracellular allosteric $\beta_{2} \mathrm{AR}$ antagonist revealed by X-ray crystal structure. Nature 548:480-484

Locksley RM (2010) Asthma and allergic inflammation. Cell 140:777-783.

Loftus PA and Wise SK (2016) Epidemiology of asthma. Curr Opin Otolaryngol Head Neck Surg 24:245-249.

Loza MJ, Foster S, Peters SP, and Penn RB (2006) Beta-agonists modulate T-cell functions via direct actions on type 1 and type 2 cells. Blood 107:2052-2060.

Loza MJ, Peters SP, Foster S, Khan IU, and Penn RB (2007) beta-Agonist enhances type 2 T-cell survival and accumulation. J Allergy Clin Immunol 119:235-244.

Lüllmann H, Ohnesorge FK, Schauwecker GC, and Wassermann O (1969) Inhibition of the actions of carbachol and DFP on guinea pig isolated atria by alkane-bisammonium compounds. Eur J Pharmacol 6:241-247.

Luttrell LM, Ferguson SS, Daaka Y, Miller WE, Maudsley S, Della Rocca GJ, Lin F, Kawakatsu H, Owada K, Luttrell DK, et al. (1999) Beta-arrestin-dependent formation of beta2 adrenergic receptor-Src protein kinase complexes. Science $\mathbf{2 8 3}$ : $655-661$.

Ly TW and Bacon KB (2005) Small-molecule CRTH2 antagonists for the treatment of allergic inflammation: an overview. Expert Opin Investig Drugs 14:769-773.

Lynch KR, O'Neill GP, Liu Q, Im DS, Sawyer N, Metters KM, Coulombe N, Abramovitz M, Figueroa DJ, Zeng Z, et al. (1999) Characterization of the human cysteinyl leukotriene CysLT1 receptor. Nature 399:789-793.

Ma P, Weichert D, Aleksandrov LA, Jensen TJ, Riordan JR, Liu X, Kobilka BK and Caffrey $\mathrm{M}$ (2017) The cubicon method for concentrating membrane proteins in the cubic mesophase. Nat Protoc 12:1745-1762.

Maclouf J, Antoine C, Henson PM, and Murphy RC (1994) Leukotriene C4 formation by transcellular biosynthesis. Ann N Y Acad Sci 714:143-150. 
Maekawa A, Austen KF, and Kanaoka Y (2002) Targeted gene disruption reveals the role of cysteinyl leukotriene 1 receptor in the enhanced vascular permeability of mice undergoing acute inflammatory responses. $\mathrm{J}$ Biol Chem 277 : 20820-20824

Maekawa A, Balestrieri B, Austen KF, and Kanaoka Y (2009) GPR17 is a negative regulator of the cysteinyl leukotriene 1 receptor response to leukotriene D4. Proc Natl Acad Sci USA 106:11685-11690.

Mahemuti G, Zhang H, Li J, Tieliwaerdi N, and Ren L (2018) Efficacy and side effects of intravenous theophylline in acute asthma: a systematic review and metaanalysis. Drug Des Devel Ther 12:99-120.

Mak JC, Baraniuk JN, and Barnes PJ (1992) Localization of muscarinic receptor subtype mRNAs in human lung. Am J Respir Cell Mol Biol 7:344-348.

Mak JC and Barnes PJ (1990) Autoradiographic visualization of muscarinic receptor subtypes in human and guinea pig lung. Am Rev Respir Dis 141:1559-1568.

Malmstrom K, Rodriguez-Gomez G, Guerra J, Villaran C, Piñeiro A, Wei LX, Seidenberg BC, and Reiss TF; Montelukast/Beclomethasone Study Group (1999) Oral montelukast, inhaled beclomethasone, and placebo for chronic asthma: a randomized, controlled trial. Ann Intern Med 130:487-495.

Manetsch M, Ramsay EE, King EM, Seidel P, Chen W, Ge Q, Hibbs DE, Newton R, and Ammit AJ (2012) Corticosteroids and $\beta_{2}$-agonists upregulate mitogenactivated protein kinase phosphatase 1: in vitro mechanisms. British Journal ofPharmacology Aug;166(7):2049-59. 22372570.

Manglik A and Kruse AC (2017) Structural basis for G protein-coupled receptor activation. Biochemistry 56:5628-5634.

Mann JS, Howarth PH, and Holgate ST (1984) Bronchoconstriction induced by ipratropium bromide in asthma: relation to hypotonicity. Br Med J (Clin Res Ed) 289:469

Marom Z, Shelhamer JH, Bach MK, Morton DR, and Kaliner M (1982) Slow-reacting substances, leukotrienes $\mathrm{C} 4$ and D4, increase the release of mucus from human airways in vitro. Am Rev Respir Dis 126:449-451.

Martí-Solano M, Schmidt D, Kolb P, and Selent J (2016) Drugging specific conformational states of GPCRs: challenges and opportunities for computational chemistry. Drug Discov Today 21:625-631.

Masureel M, Zou Y, Picard LP, van der Westhuizen E, Mahoney JP, Rodrigues JPGLM, Mildorf TJ, Dror RO, Shaw DE, Bouvier M, et al. (2018) Structural insights into binding specificity, efficacy and bias of a $\beta_{2} \mathrm{AR}$ partial agonist. Nat Chem Biol 14:1059-1066.

Mathew A, MacLean JA, DeHaan E, Tager AM, Green FH, and Luster AD (2001) Signal transducer and activator of transcription 6 controls chemokine production and T helper cell type 2 cell trafficking in allergic pulmonary inflammation. J Exp Med 193:1087-1096.

Mathiesen JM, Christopoulos A, Ulven T, Royer JF, Campillo M, Heinemann A Pardo L, and Kostenis E (2006) On the mechanism of interaction of potent surmountable and insurmountable antagonists with the prostaglandin D2 receptor CRTH2. Mol Pharmacol 69:1441-1453.

Matsuda A, Fukuda S, Matsumoto K, and Saito H (2008) Th1/Th2 cytokines reciprocally regulate in vitro pulmonary angiogenesis via CXC chemokine synthesis. Am J Respir Cell Mol Biol 38:168-175.

Matsuda K, Makhay M, Johnson K, and Iwaki Y (2012) Evaluation of bedoradrine sulfate (MN-221), a novel, highly selective beta2-adrenergic receptor agonist for the treatment of asthma via intravenous infusion. J Asthma 49:1071-1078.

Matsuoka T, Hirata M, Tanaka H, Takahashi Y, Murata T, Kabashima K, Sugimoto Y, Kobayashi T, Ushikubi F, Aze Y, et al. (2000) Prostaglandin D2 as a mediator of allergic asthma. Science 287:2013-2017.

Matsuse H and Kohno S (2014) Leukotriene receptor antagonists pranlukast and montelukast for treating asthma. Expert Opin Pharmacother 15:353-363.

Maussang D, Mujić-Delić A, Descamps FJ, Stortelers C, Vanlandschoot P, Stigter-van Walsum M, Vischer HF, van Roy M, Vosjan M, Gonzalez-Pajuelo M, et al. (2013) Llama-derived single variable domains (nanobodies) directed against chemokine receptor CXCR7 reduce head and neck cancer cell growth in vivo. J Biol Chem 288:29562-29572.

McAlees JW and Sanders VM (2009) Hematopoietic protein tyrosine phosphatase mediates beta2-adrenergic receptor-induced regulation of p38 mitogen-activated protein kinase in B lymphocytes. Mol Cell Biol 29:675-686.

McCrea KE and Hill SJ (1993) Salmeterol, a long-acting beta 2-adrenoceptor agonist mediating cyclic AMP accumulation in a neuronal cell line. Br J Pharmacol 110 619-626.

McIvor RA, Kaplan A, Koch C, and Sampalis JS (2009) Montelukast as an alternative to low-dose inhaled corticosteroids in the management of mild asthma (the SIMPLE trial): an open-label effectiveness trial. Can Respir $J 16$ (Suppl A):11A-21A.

McKinley L, Alcorn JF, Peterson A, Dupont RB, Kapadia S, Logar A, Henry A, Irvin CG, Piganelli JD, Ray A, et al. (2008) TH17 cells mediate steroid-resistant airway inflammation and airway hyperresponsiveness in mice. $J$ Immunol 181 4089-4097.

Mellor EA, Austen KF, and Boyce JA (2002) Cysteinyl leukotrienes and uridine diphosphate induce cytokine generation by human mast cells through an interleukin 4-regulated pathway that is inhibited by leukotriene receptor antagonists. J Exp Med 195:583-592.

Mellor EA, Frank N, Soler D, Hodge MR, Lora JM, Austen KF, and Boyce JA (2003) Expression of the type 2 receptor for cysteinyl leukotrienes (CysLT2R) by human mast cells: functional distinction from CysLT1R. Proc Natl Acad Sci USA 100: 11589-11593.

Middleton E Jr and Finke SR (1968) Metabolic response to epinephrine in bronchial asthma. J Allergy 42:288-299.

Miller D, Wood C, Bateman E, LaForce C, Blatchford J, Hilbert J, Gupta A, and Fowler A (2017) A randomized study of BI 671800, a CRTH2 antagonist, as add-on therapy in poorly controlled asthma. Allergy Asthma Proc 38:157-164.

Min B, Prout M, Hu-Li J, Zhu J, Jankovic D, Morgan ES, Urban JF Jr, Dvorak AM, Finkelman FD, LeGros G, et al. (2004) Basophils produce IL-4 and accumulate in tissues after infection with a Th2-inducing parasite. J Exp Med 200:507-517.
Miraldi E, Masti A, Ferri S, and Barni Comparini I (2001) Distribution of hyoscyamine and scopolamine in Datura stramonium. Fitoterapia 72:644-648.

Mitchelson F (1988) Muscarinic receptor differentiation. Pharmacol Ther 37: $357-423$

Miyahara N, Takeda K, Miyahara S, Matsubara S, Koya T, Joetham A, Krishnan E, Dakhama A, Haribabu B, and Gelfand EW (2005) Requirement for leukotriene B4 receptor 1 in allergen-induced airway hyperresponsiveness. Am J Respir Crit Care Med 172:161-167.

Monaco TJ and Hanania NA (2017) Emerging inhaled long-acting beta-2 adrenoceptor agonists for the treatment of COPD. Expert Opin Emerg Drugs 22:285-299.

Montuschi P and Ciabattoni G (2015) Bronchodilating drugs for chronic obstructive pulmonary disease: current status and future trends. J Med Chem 58:4131-4164.

Moore RH, Millman EE, Godines V, Hanania NA, Tran TM, Peng H, Dickey BF, Knoll BJ, and Clark RB (2007) Salmeterol stimulation dissociates beta2-adrenergic receptor phosphorylation and internalization. Am J Respir Cell Mol Biol 36: $254-261$.

Moos MP, Mewburn JD, Kan FW, Ishii S, Abe M, Sakimura K, Noguchi K, Shimizu T, and Funk CD (2008) Cysteinyl leukotriene 2 receptor-mediated vascular permeability via transendothelial vesicle transport. FASEB $J$ 22:4352-4362.

Morales DR, Lipworth BJ, Donnan PT, Jackson C, and Guthrie B (2017) Respiratory effect of beta-blockers in people with asthma and cardiovascular disease: population-based nested case control study. BMC Med 15:18.

Morgan SJ, Deshpande DA, Tiegs BC, Misior AM, Yan H, Hershfeld AV, Rich TC Panettieri RA, An SS, and Penn RB (2014) $\beta$-Agonist-mediated relaxation of airway smooth muscle is protein kinase A-dependent. J Biol Chem 289:23065-23074.

Moro K, Yamada T, Tanabe M, Takeuchi T, Ikawa T, Kawamoto H, Furusawa J, Ohtani M, Fujii H, and Koyasu S (2010) Innate production of $\mathrm{T}(\mathrm{H}) 2$ cytokines by adipose tissue-associated c-Kit(+)Sca-1(+) lymphoid cells. Nature 463:540-544.

Morris HR, Taylor GW, Piper PJ, Sirois P, and Tippins JR (1978) Slow-reacting substance of anaphylaxis: purification and characterisation. FEBS Lett 87: 203-206.

Morris HR, Taylor GW, Piper PJ, and Tippins JR (1980) Structure of slow-reacting substance of anaphylaxis from guinea-pig lung. Nature 285:104-106.

Moulton BC and Fryer AD (2011) Muscarinic receptor antagonists, from folklore to pharmacology; finding drugs that actually work in asthma and COPD. $\mathrm{Br}$ $J$ Pharmacol 163:44-52.

Mullane K and Williams M (2014) Animal models of asthma: reprise or reboot? Biochem Pharmacol 87:131-139.

Munoz NM, Vita AJ, Neeley SP, McAllister K, Spaethe SM, White SR, and Leff AR (1994) Beta adrenergic modulation of formyl-methionine-leucine-phenylalaninestimulated secretion of eosinophil peroxidase and leukotriene C4. J Pharmacol Exp Ther 268:139-143.

Murray JJ, Tonnel AB, Brash AR, Roberts LJ II, Gosset P, Workman R, Capron A and Oates JA (1986) Release of prostaglandin D2 into human airways during acute antigen challenge. $N$ Engl J Med 315:800-804.

Mustafa SJ, Nadeem A, Fan M, Zhong H, Belardinelli L, and Zeng D (2007) Effect of a specific and selective $\mathrm{A}(2 \mathrm{~B})$ adenosine receptor antagonist on adenosine agonist AMP and allergen-induced airway responsiveness and cellular influx in a mouse model of asthma. J Pharmacol Exp Ther 320:1246-1251.

Muyldermans S (2013) Nanobodies: natural single-domain antibodies. Annu Rev Biochem 82:775-797.

Myou S, Fujimura M, Kamio Y, Kita T, Katayama N, Abo M, Yoshimi Y, Nishitsuji M, Nomura S, Hashimoto T, et al. (2001) Effect of a cysteinyl leukotriene antagonist, pranlukast hydrate, on acetaldehyde-induced bronchoconstriction in asthmatic patients. Prostaglandins Leukot Essent Fatty Acids 65:41-44.

Mysinger MM, Weiss DR, Ziarek JJ, Gravel S, Doak AK, Karpiak J, Heveker N, Shoichet BK, and Volkman BF (2012) Structure-based ligand discovery for the protein-protein interface of chemokine receptor CXCR4. Proc Natl Acad Sci USA 109:5517-5522.

Nagar B, Bornmann WG, Pellicena P, Schindler T, Veach DR, Miller WT, Clarkson B, and Kuriyan J (2002) Crystal structures of the kinase domain of c-Abl in complex with the small molecule inhibitors PD173955 and imatinib (STI-571). Cancer Res 62:4236-4243.

Nagata K and Hirai H (2003) The second PGD(2) receptor CRTH2: structure, properties, and functions in leukocytes. Prostaglandins Leukot Essent Fatty Acids 69: $169-177$.

Nagata K, Hirai H, Tanaka K, Ogawa K, Aso T, Sugamura K, Nakamura M, and Takano S (1999) CRTH2, an orphan receptor of T-helper-2-cells, is expressed on basophils and eosinophils and responds to mast cell-derived factor(s). FEBS Lett 459:195-199.

Nagata M, Saito K, Tsuchiya K, and Sakamoto Y (2002) Leukotriene D4 upregulates eosinophil adhesion via the cysteinyl leukotriene 1 receptor. J Allergy Clin Immunol 109:676-680.

Nair P, Gaga M, Zervas E, Alagha K, Hargreave FE, O’Byrne PM, Stryszak P, Gann L, Sadeh J, and Chanez P; Study Investigators (2012) Safety and efficacy of a CXCR2 antagonist in patients with severe asthma and sputum neutrophils: a randomized, placebo-controlled clinical trial. Clin Exp Allergy 42:1097-1103.

Nakagawa N, Obata T, Kobayashi T, Okada Y, Nambu F, Terawaki T, and Aishita H (1992) In vivo pharmacologic profile of ONO-1078: a potent, selective and orally active peptide leukotriene (LT) antagonist. Jpn J Pharmacol 60:217-225.

Nakagawa N, Obata T, Kobayashi T, Okada Y, Nambu F, Terawaki T, Furuya T, Muryobayashi K, Sawada M, and Aishita H (1993) Effect of a peptide leukotriene receptor antagonist, ONO-1078, on guinea-pig models of asthma. Eur J Pharmaco 235:211-219.

Nakai H, Konno M, Kosuge S, Sakuyama S, Toda M, Arai Y, Obata T, Katsube N, Miyamoto T, Okegawa T, et al. (1988) New potent antagonists of leukotrienes C4 and D4. 1. Synthesis and structure-activity relationships. J Med Chem 31:84-91.

Nakamura Y, Hoshino M, Sim JJ, Ishii K, Hosaka K, and Sakamoto T (1998) Effect of the leukotriene receptor antagonist pranlukast on cellular infiltration in the bronchial mucosa of patients with asthma. Thorax 53:835-841. 
Naline E, Zhang Y, Qian Y, Mairon N, Anderson GP, Grandordy B, and Advenier C (1994) Relaxant effects and durations of action of formoterol and salmeterol on the isolated human bronchus. Eur Respir J 7:914-920.

National Asthma Education and Prevention Program (2007) Expert Panel Report 3 (EPR-3): guidelines for the diagnosis and management of asthma-summary report 2007. J Allergy Clin Immunol 120 (Suppl):S94-S138.

Neill DR, Wong SH, Bellosi A, Flynn RJ, Daly M, Langford TK, Bucks C, Kane CM, Fallon PG, Pannell R, et al. (2010) Nuocytes represent a new innate effector leukocyte that mediates type-2 immunity. Nature 464:1367-1370.

Nelson HS, Nathan RA, Kalberg C, Yancey SW, and Rickard KA (2001) Comparison of inhaled salmeterol and oral zafirlukast in asthmatic patients using concomitant inhaled corticosteroids. MedGenMed 3:3.

Nelson RP Jr, DiNicolo R, Fernández-Caldas E, Seleznick MJ, Lockey RF, and Good RA (1996) Allergen-specific IgE levels and mite allergen exposure in children with acute asthma first seen in an emergency department and in nonasthmatic control subjects. J Allergy Clin Immunol 98:258-263.

Neumann D, Schneider EH, and Seifert R (2014) Analysis of histamine receptor knockout mice in models of inflammation. J Pharmacol Exp Ther 348:2-11.

Nguyen LP, Al-Sawalha NA, Parra S, Pokkunuri I, Omoluabi O, Okulate AA, Windham Li E, Hazen M, Gonzalez-Granado JM, Daly CJ, et al. (2017) $\beta_{2}$-Adrenoceptor signaling in airway epithelial cells promotes eosinophilic inflammation, mucous metaplasia, and airway contractility. Proc Natl Acad Sci USA 114:E9163-E9171.

Nials AT, Sumner MJ, Johnson M, and Coleman RA (1993) Investigations into factors determining the duration of action of the beta 2-adrenoceptor agonist, salmeterol. Br J Pharmacol 108:507-515.

Nials AT and Uddin S (2008) Mouse models of allergic asthma: acute and chronic allergen challenge. Dis Model Mech 1:213-220.

Nishi K, Fujimura M, Myou S, Ooka T, Sakamoto S, Saitou M, Kasahara K, and Matsuda T (1993) Comparison of the bronchodilator activities of oxitropium bromide, fenoterol, and their combination in patients with chronic obstructive pulmonary disease and bronchial asthma. Clin Auton Res 3:41-44.

Norman P (2014) Update on the status of DP2 receptor antagonists; from proof of concept through clinical failures to promising new drugs. Expert Opin Investig Drugs 23:55-66.

Norris Reinero CR, Decile KC, Berghaus RD, Williams KJ, Leutenegger CM, Walby WF, Schelegle ES, Hyde DM, and Gershwin LJ (2004) An experimental model of allergic asthma in cats sensitized to house dust mite or bermuda grass allergen. Int Arch Allergy Immunol 135:117-131.

Nouri-Aria KT, Irani AM, Jacobson MR, O’Brien F, Varga EM, Till SJ, Durham SR, and Schwartz LB (2001) Basophil recruitment and IL-4 production during human allergen-induced late asthma. J Allergy Clin Immunol 108:205-211.

Nurmagambetov T, Kuwahara R, and Garbe P (2018) The economic burden of asthma in the United States, 2008-2013. Ann Am Thorac Soc 15:348-356.

Obase Y, Shimoda T, Tomari S, Mitsuta K, Fukushima C, Kawano T, Matsuse H, and Kohno S (2001) Effects of pranlukast on aspirin-induced bronchoconstriction: differences in chemical mediators between aspirin-intolerant and tolerant asthmatic patients. Ann Allergy Asthma Immunol 87:74-79.

Obase Y, Shimoda T, Tomari SY, Mitsuta K, Kawano T, Matsuse H, and Kohno S (2002) Effects of pranlukast on chemical mediators in induced sputum on provocation tests in atopic and aspirin-intolerant asthmatic patients. Chest 121:143-150.

Obata T, Kobayashi T, Okada Y, Nakagawa N, Terawaki T, and Aishita H (1992) Effect of a peptide leukotriene antagonist, ONO-1078 on antigen-induced airway microvascular leakage in actively sensitized guinea pigs. Life Sci 51:1577-1583.

Obata T, Nambu F, Kitagawa T, Terashima H, Toda M, Okegawa T, and Kawasaki A (1987) ONO-1078: an antagonist of leukotrienes. Adv Prostaglandin Thromboxane Leukot Res 17A:540-543.

O'Byrne PM, Bisgaard H, Godard PP, Pistolesi M, Palmqvist M, Zhu Y, Ekström T, and Bateman ED (2005) Budesonide/formoterol combination therapy as both maintenance and reliever medication in asthma. Am J Respir Crit Care Med 171:129-136.

O’Byrne PM, Metev H, Puu M, Richter K, Keen C, Uddin M, Larsson B, Cullberg M, and Nair P (2016) Efficacy and safety of a CXCR2 antagonist, AZD5069, in patients with uncontrolled persistent asthma: a randomised, double-blind, placebocontrolled trial. Lancet Respir Med 4:797-806.

Ochensberger B, Tassera L, Bifrare D, Rihs S, and Dahinden CA (1999) Regulation of cytokine expression and leukotriene formation in human basophils by growth factors, chemokines and chemotactic agonists. Eur J Immunol 29:11-22.

Oguma T, Palmer LJ, Birben E, Sonna LA, Asano K, and Lilly CM (2004) Role of prostanoid DP receptor variants in susceptibility to asthma. $N$ Engl J Med 351 $1752-1763$.

Ohbayashi H, Shibata N, Hirose T, and Adachi M (2009) Additional effects of pranlukast in salmeterol/fluticasone combination therapy for the asthmatic distal airway in a randomized crossover study. Pulm Pharmacol Ther 22:574-579.

Olah ME and Stiles GL (1995) Adenosine receptor subtypes: characterization and therapeutic regulation. Annu Rev Pharmacol Toxicol 35:581-606.

Olenchock BA, Fonarow GG, Pan W, Hernandez A, and Cannon CP; Get With The Guidelines Steering Committee (2009) Current use of beta blockers in patients with reactive airway disease who are hospitalized with acute coronary syndromes. Am J Cardiol 103:295-300.

Oliphant CJ, Hwang YY, Walker JA, Salimi M, Wong SH, Brewer JM, Englezakis A Barlow JL, Hams E, Scanlon ST, et al. (2014) MHCII-mediated dialog between group 2 innate lymphoid cells and CD4 $(+) \mathrm{T}$ cells potentiates type 2 immunity and promotes parasitic helminth expulsion. Immunity 41:283-295.

O'Reilly M, Alpert R, Jenkinson S, Gladue RP, Foo S, Trim S, Peter B, Trevethick M, and Fidock M (2002) Identification of a histamine H4 receptor on human eosinophils--role in eosinophil chemotaxis. J Recept Signal Transduct Res 22:431-448.

Ortega VE, Hawkins GA, Moore WC, Hastie AT, Ampleford EJ, Busse WW, Castro M, Chardon D, Erzurum SC, Israel E, et al. (2014) Effect of rare variants in ADRB2 on risk of severe exacerbations and symptom control during longacting $\beta$ agonist treatment in a multiethnic asthma population: a genetic study. Lancet Respir Med 2:204-213.
Padrid P (1992) Chronic lower airway disease in the dog and cat. Probl Vet Med 4: 320-344.

Padrid P (2000) Feline asthma: diagnosis and treatment. Vet Clin North Am Small Anim Pract 30:1279-1293.

Page C and Cazzola M (2014) Bifunctional drugs for the treatment of asthma and chronic obstructive pulmonary disease. Eur Respir $J$ 44:475-482.

Palczewski K, Kumasaka T, Hori T, Behnke CA, Motoshima H, Fox BA, Le Trong I, Teller DC, Okada T, Stenkamp RE, et al. (2000) Crystal structure of rhodopsin: a G protein-coupled receptor. Science 289:739-745.

Palmqvist M, Ibsen T, Mellén A, and Lötvall J (1999) Comparison of the relative efficacy of formoterol and salmeterol in asthmatic patients. Am J Respir Crit Care Med 160:244-249.

Palmqvist M, Persson G, Lazer L, Rosenborg J, Larsson P, and Lötvall J (1997) Inhaled dry-powder formoterol and salmeterol in asthmatic patients: onset of action, duration of effect and potency. Eur Respir J 10:2484-2489.

Pandya D, Puttanna A, and Balagopal V (2014) Systemic effects of inhaled corticosteroids: an overview. Open Respir Med J 8:59-65.

Papi A, Saetta M, and Fabbri L (2017) Severe asthma: phenotyping to endotyping or vice versa? Eur Respir J 49:1700053.

Pare PD, Roberts CR, Bai TR, and Wiggs BJ (1997) The functional consequences of airway remodeling in asthma. Monaldi Arch Chest Dis 52:589-596.

Paruchuri S, Tashimo H, Feng C, Maekawa A, Xing W, Jiang Y, Kanaoka Y, Conley $\mathrm{P}$, and Boyce JA (2009) Leukotriene E4-induced pulmonary inflammation is mediated by the P2Y12 receptor. $J$ Exp Med 206:2543-2555.

Paul WE and Zhu J (2010) How are T(H)2-type immune responses initiated and amplified? Nat Rev Immunol 10:225-235.

Pavord ID, Beasley R, Agusti A, Anderson GP, Bel E, Brusselle G, Cullinan P, Custovic A, Ducharme FM, Fahy JV, et al. (2018) After asthma: redefining airways diseases. Lancet 391:350-400.

Pearlman DS, Lampl KL, Dowling PJ Jr, Miller CJ, and Bonuccelli CM (2000) Effectiveness and tolerability of zafirlukast for the treatment of asthma in children. Clin Ther 22:732-747.

Pease JE (2011) Targeting chemokine receptors in allergic disease. Biochem J 434 11-24.

Pease JE and Horuk R (2014) Recent progress in the development of antagonists to the chemokine receptors CCR3 and CCR4. Expert Opin Drug Discov 9: $467-483$.

Pedersen KE, Bochner BS, and Undem BJ (1997) Cysteinyl leukotrienes induce P-selectin expression in human endothelial cells via a non-CysLT1 receptormediated mechanism. J Pharmacol Exp Ther 281:655-662.

Peebles RS Jr (2019) Prostaglandins in asthma and allergic diseases. Pharmacol Ther 193:1-19.

Pelaia G, Vatrella A, and Maselli R (2012) The potential of biologics for the treatment of asthma. Nat Rev Drug Discov 11:958-972.

Penn RB (2008) Embracing emerging paradigms of G protein-coupled receptor agonism and signaling to address airway smooth muscle pathobiology in asthma. Naunyn Schmiedebergs Arch Pharmacol 378:149-169.

Penn RB (2014) Far from “disappointing”. Am J Respir Crit Care Med 189:360.

Penn RB, Bond RA, and Walker JK (2014) GPCRs and arrestins in airways: implications for asthma. Handb Exp Pharmacol 219:387-403.

Pera T and Penn RB (2016) Bronchoprotection and bronchorelaxation in asthma: new targets, and new ways to target the old ones. Pharmacol Ther 164:82-96.

Perlikos F, Hillas G, and Loukides S (2016) Phenotyping and endotyping asthma based on biomarkers. Curr Top Med Chem 16:1582-1586.

Peters-Golden M, Gleason MM, and Togias A (2006) Cysteinyl leukotrienes: multifunctional mediators in allergic rhinitis. Clin Exp Allergy 36:689-703.

Pettipher R (2008) The roles of the prostaglandin D(2) receptors DP(1) and CRTH2 in promoting allergic responses. Br J Pharmacol 153 (Suppl 1):S191-S199.

Pettipher R, Hunter MG, Perkins CM, Collins LP, Lewis T, Baillet M, Steiner J, Bell $\mathrm{J}$, and Payton MA (2014) Heightened response of eosinophilic asthmatic patients to the CRTH2 antagonist OC000459. Allergy 69:1223-1232.

Pettipher R, Vinall SL, Xue L, Speight G, Townsend ER, Gazi L, Whelan CJ, Armer RE, Payton MA, and Hunter MG (2012) Pharmacologic profile of OC000459, a potent, selective, and orally active D prostanoid receptor 2 antagonist that inhibits mast cell-dependent activation of $\mathrm{T}$ helper 2 lymphocytes and eosinophils. J Pharmacol Exp Ther 340:473-482.

Pettipher R and Whittaker M (2012) Update on the development of antagonists of chemoattractant receptor-homologous molecule expressed on Th2 cells (CRTH2): from lead optimization to clinical proof-of-concept in asthma and allergic rhinitis. J Med Chem 55:2915-2931.

Peyrassol X, Laeremans T, Gouwy M, Lahura V, Debulpaep M, Van Damme J, Steyaert J, Parmentier M, and Langer I (2016) Development by genetic immunization of monovalent antibodies (nanobodies) behaving as antagonists of the human ChemR23 receptor. J Immunol 196:2893-2901.

Pollock K and Creba J (1990) Leukotriene D4 induced calcium changes in U937 cells may utilize mechanisms additional to inositol phosphate production that are pertussis toxin insensitive but are blocked by phorbol myristate acetate. Cell Signal 2 563-568.

Pollock M, Sinha IP, Hartling L, Rowe BH, Schreiber S, and Fernandes RM (2017) Inhaled short-acting bronchodilators for managing emergency childhood asthma: an overview of reviews. Allergy 72:183-200.

Polosa R (2002) Adenosine-receptor subtypes: their relevance to adenosine-mediated responses in asthma and chronic obstructive pulmonary disease. Eur Respir J 20: $488-496$.

Pongratz G, McAlees JW, Conrad DH, Erbe RS, Haas KM, and Sanders VM (2006) The level of IgE produced by a B cell is regulated by norepinephrine in a p38 MAPK- and CD23-dependent manner. J Immunol 177:2926-2938.

Prevete N, Liotti F, Marone G, Melillo RM, and de Paulis A (2015) Formyl peptide receptors at the interface of inflammation, angiogenesis and tumor growth. Pharmacol Res 102:184-191. 
Price AE, Liang HE, Sullivan BM, Reinhardt RL, Eisley CJ, Erle DJ, and Locksley RM (2010) Systemically dispersed innate IL-13-expressing cells in type 2 immunity. Proc Natl Acad Sci USA 107:11489-11494.

Price D, Popov TA, Bjermer L, Lu S, Petrovic R, Vandormael K, Mehta A, Strus JD, Polos PG, and Philip G (2013) Effect of montelukast for treatment of asthma in cigarette smokers. J Allergy Clin Immunol 131:763-771.

Profita M, Bonanno A, Siena L, Bruno A, Ferraro M, Montalbano AM, Albano GD, Riccobono L, Casarosa P, Pieper MP, et al. (2009) Smoke, choline acetyltransferase, muscarinic receptors, and fibroblast proliferation in chronic obstructive pulmonary disease. J Pharmacol Exp Ther 329:753-763.

Proska J and Tucek S (1994) Mechanisms of steric and cooperative actions of alcuronium on cardiac muscarinic acetylcholine receptors. Mol Pharmacol 45: 709-717.

Raabe CA, Gröper J, and Rescher U (2019) Biased perspectives on formyl peptide receptors. Biochim Biophys Acta Mol Cell Res 1866:305-316.

Rabe KF, Hurd S, Anzueto A, Barnes PJ, Buist SA, Calverley P, Fukuchi Y, Jenkins C, Rodriguez-Roisin R, van Weel C, et al.; Global Initiative for Chronic Obstructive Lung Disease (2007) Global strategy for the diagnosis, management, and prevention of chronic obstructive pulmonary disease: GOLD executive summary. Am $J$ Respir Crit Care Med 176:532-555.

Radosa J, Dyck W, Goerdt S, and Kurzen H (2011) The cholinergic system in guttate psoriasis with special reference to mast cells. Exp Dermatol 20:677-679.

Rael EL and Lockey RF (2011) Interleukin-13 signaling and its role in asthma. World Allergy Organ J 4:54-64.

Raissy HH, Harkins M, Kelly F, and Kelly HW (2008) Pretreatment with albuterol versus montelukast for exercise-induced bronchospasm in children. Pharmacotherapy 28:287-294.

Rajapaksa KS, Huang T, Sharma N, Liu S, Solon M, Reyes A, Paul S, Yee A, Tao J, Chalasani S, et al. (2016) Preclinical safety profile of a depleting antibody against CRTh2 for asthma: well tolerated despite unexpected CRTh2 expression on vascular pericytes in the central nervous system and gastric mucosa. Toxicol Sci 152: 72-84.

Rasmussen SG, Choi HJ, Fung JJ, Pardon E, Casarosa P, Chae PS, Devree BT, Rosenbaum DM, Thian FS, Kobilka TS, et al. (2011a) Structure of a nanobodystabilized active state of the $\beta(2)$ adrenoceptor. Nature 469:175-180.

Rasmussen SG, Choi HJ, Rosenbaum DM, Kobilka TS, Thian FS, Edwards PC, Burghammer M, Ratnala VR, Sanishvili R, Fischetti RF, et al. (2007) Crystal structure of the human beta2 adrenergic G-protein-coupled receptor. Nature $\mathbf{4 5 0}$ 383-387.

Rasmussen SG, DeVree BT, Zou Y, Kruse AC, Chung KY, Kobilka TS, Thian FS, Chae PS, Pardon E, Calinski D, et al. (2011b) Crystal structure of the $\beta 2$ adrenergic receptor-Gs protein complex. Nature 477:549-555.

Ravasi S, Capra V, Panigalli T, Rovati GE, and Nicosia S (2002) Pharmacological differences among CysLT(1) receptor antagonists with respect to LTC(4) and LTD(4) in human lung parenchyma. Biochem Pharmacol 63:1537-1546.

Ravasi S, Citro S, Viviani B, Capra V, and Rovati GE (2006) CysLT1 receptor-induced human airway smooth muscle cells proliferation requires ROS generation, EGF receptor transactivation and ERK1/2 phosphorylation. Respir Res 7:42.

Reddel HK, Bateman ED, Becker A, Boulet LP, Cruz AA, Drazen JM, Haahtela T, Hurd SS, Inoue H, de Jongste JC, et al. (2015) A summary of the new GINA strategy: a roadmap to asthma control. Eur Respir J 46:622-639.

Reiss TF, Chervinsky P, Dockhorn RJ, Shingo S, Seidenberg B, and Edwards TB; Montelukast Clinical Research Study Group (1998) Montelukast, a once-daily leukotriene receptor antagonist, in the treatment of chronic asthma: a multicenter, randomized, double-blind trial. Arch Intern Med 158:1213-1220.

Reiss TF, Sorkness CA, Stricker W, Botto A, Busse WW, Kundu S, and Zhang J (1997) Effects of montelukast (MK-0476); a potent cysteinyl leukotriene receptor antagonist, on bronchodilation in asthmatic subjects treated with and without inhaled corticosteroids. Thorax 52:45-48.

Restrepo RD (2007) Use of inhaled anticholinergic agents in obstructive airway disease. Respir Care 52:833-851.

Ricklefs I, Barkas I, Duvall MG, Cernadas M, Grossman NL, Israel E, Bleecker ER, Castro M, Erzurum SC, Fahy JV, et al.; National Heart Lung and Blood Institute's Severe Asthma Research Program-3 Investigators (2017) ALX receptor ligands define a biochemical endotype for severe asthma. JCI Insight 2:e93534

Ring AM, Manglik A, Kruse AC, Enos MD, Weis WI, Garcia KC, and Kobilka BK (2013) Adrenaline-activated structure of $\beta 2$-adrenoceptor stabilized by an engineered nanobody. Nature 502:575-579.

Ritchie AI, Singanayagam A, Wiater E, Edwards MR, Montminy M, and Johnston SL (2018) $\beta_{2}$-agonists enhance asthma-relevant inflammatory mediators in human airway epithelial cells. Am J Respir Cell Mol Biol 58:128-132.

Robinson D, Humbert M, Buhl R, Cruz AA, Inoue H, Korom S, Hanania NA, and Nair $\mathrm{P}$ (2017) Revisiting type 2-high and type 2-low airway inflammation in asthma: current knowledge and therapeutic implications. Clin Exp Allergy 47:161-175.

Roeder T, Isermann K, and Kabesch M (2009) Drosophila in asthma research. Am $J$ Respir Crit Care Med 179:979-983.

Roffel AF, Elzinga CR, Van Amsterdam RG, De Zeeuw RA, and Zaagsma J (1988) Muscarinic M2 receptors in bovine tracheal smooth muscle: discrepancies between binding and function. Eur J Pharmacol 153:73-82.

Roffel AF, Elzinga CR, and Zaagsma J (1990) Muscarinic M3 receptors mediate contraction of human central and peripheral airway smooth muscle. Pulm Pharmacol 3:47-51.

Rolla G and Brussino L (2018) Single-inhaler triple versus dual therapy in patients with COPD. $N$ Engl J Med 379:590-591.

Roscioni SS, Elzinga CR, and Schmidt M (2008) Epac: effectors and biological functions. Naunyn Schmiedebergs Arch Pharmacol 377:345-357.

Rosenbaum DM, Cherezov V, Hanson MA, Rasmussen SG, Thian FS, Kobilka TS Choi HJ, Yao XJ, Weis WI, Stevens RC, et al. (2007) GPCR engineering yields highresolution structural insights into beta2-adrenergic receptor function. Science $\mathbf{3 1 8}$ $1266-1273$
Rosenbaum DM, Zhang C, Lyons JA, Holl R, Aragao D, Arlow DH, Rasmussen SG, Choi HJ, Devree BT, Sunahara RK, et al. (2011) Structure and function of an irreversible agonist- $\beta(2)$ adrenoceptor complex. Nature 469:236-240.

Rossi D and Zlotnik A (2000) The biology of chemokines and their receptors. Annu Rev Immunol 18:217-242.

Royer JF, Schratl P, Lorenz S, Kostenis E, Ulven T, Schuligoi R, Peskar BA, and Heinemann A (2007) A novel antagonist of CRTH2 blocks eosinophil release from bone marrow, chemotaxis and respiratory burst. Allergy 62:1401-1409.

Ryzhov S, Goldstein AE, Matafonov A, Zeng D, Biaggioni I, and Feoktistov I (2004) Adenosine-activated mast cells induce IgE synthesis by B lymphocytes: an A2Bmediated process involving Th2 cytokines IL-4 and IL-13 with implications for asthma. J Immunol 172:7726-7733.

Sagara H, Yukawa T, Kashima R, Okada T, and Fukuda T (2009) Effects of pranlukast hydrate on airway hyperresponsiveness in non-asthmatic patients with Japanese cedar pollinosis. Allergol Int 58:277-287.

Salmon M, Luttmann MA, Foley JJ, Buckley PT, Schmidt DB, Burman M, Webb EF, DeHaas CJ, Kotzer CJ, Barrett VJ, et al. (2013) Pharmacological characterization of GSK573719 (umeclidinium): a novel, long-acting, inhaled antagonist of the muscarinic cholinergic receptors for treatment of pulmonary diseases. $J$ Pharmacol Exp Ther 345:260-270.

Salpeter SR, Ormiston TM, and Salpeter EE (2004) Cardiovascular effects of betaagonists in patients with asthma and COPD: a meta-analysis. Chest 125: 2309-2321.

Samuelsson B (1983) Leukotrienes: mediators of immediate hypersensitivity reactions and inflammation. Science 220:568-575.

Sanders VM (2012) The beta2-adrenergic receptor on T and B lymphocytes: do we understand it yet? Brain Behav Immun 26:195-200.

Sanders VM, Baker RA, Ramer-Quinn DS, Kasprowicz DJ, Fuchs BA, and Street NE (1997) Differential expression of the beta2-adrenergic receptor by Th1 and Th2 clones: implications for cytokine production and B cell help. $J$ Immunol 158: $4200-4210$.

Sandham DA, Barker L, Brown L, Brown Z, Budd D, Charlton SJ, Chatterjee D, Cox B, Dubois G, Duggan N, et al. (2017) Discovery of fevipiprant (NVP-QAW039) a potent and selective $\mathrm{DP}_{2}$ receptor antagonist for treatment of asthma. ACS Med Chem Lett 8:582-586.

Santos R, Ursu O, Gaulton A, Bento AP, Donadi RS, Bologa CG, Karlsson A, AlLazikani B, Hersey A, Oprea TI, et al. (2017) A comprehensive map of molecular drug targets. Nat Rev Drug Discov 16:19-34.

Scheerlinck JP, Snibson KJ, Bowles VM, and Sutton P (2008) Biomedical applications of sheep models: from asthma to vaccines. Trends Biotechnol 26:259-266.

Schmidt JA, Bell FM, Akam E, Marshall C, Dainty IA, Heinemann A, Dougall IG, Bonnert RV, and Sargent CA (2013) Biochemical and pharmacological characterization of AZD1981, an orally available selective DP2 antagonist in clinical development for asthma. Br J Pharmacol 168:1626-1638.

Schoch GA, Yano JK, Sansen S, Dansette PM, Stout CD, and Johnson EF (2008) Determinants of cytochrome P450 2C8 substrate binding: structures of complexes with montelukast, troglitazone, felodipine, and 9-cis-retinoic acid. $J$ Biol Chem 283:17227-17237.

Scholler P, Nevoltris D, de Bundel D, Bossi S, Moreno-Delgado D, Rovira X, Møller TC, El Moustaine D, Mathieu M, Blanc E, et al. (2017) Allosteric nanobodies uncover a role of hippocampal mGlu2 receptor homodimers in contextual fear consolidation. Nat Commun 8:1967.

Schoors DF, De Smet M, Reiss T, Margolskee D, Cheng H, Larson P, Amin R and Somers G (1995) Single dose pharmacokinetics, safety and tolerability of MK0476, a new leukotriene D4-receptor antagonist, in healthy volunteers. Br J Clin Pharmacol 40:277-280.

Schramm CM, Arjona NC, and Grunstein MM (1995) Role of muscarinic M2 receptors in regulating beta-adrenergic responsiveness in maturing rabbit airway smooth muscle. Am J Physiol 269:L783-L790.

Schuh JM, Blease K, and Hogaboam CM (2002) CXCR2 is necessary for the development and persistence of chronic fungal asthma in mice. J Immunol 168:1447-1456.

Scola AM, Loxham M, Charlton SJ, and Peachell PT (2009) The long-acting betaadrenoceptor agonist, indacaterol, inhibits IgE-dependent responses of human lung mast cells. Br J Pharmacol 158:267-276.

Scott JP and Peters-Golden M (2013) Antileukotriene agents for the treatment of lung disease. Am J Respir Crit Care Med 188:538-544.

Scow DT, Luttermoser GK, and Dickerson KS (2007) Leukotriene inhibitors in the treatment of allergy and asthma. Am Fam Physician 75:65-70.

Seifert R, Strasser A, Schneider EH, Neumann D, Dove S, and Buschauer A (2013) Molecular and cellular analysis of human histamine receptor subtypes. Trends Pharmacol Sci 34:33-58.

Sekioka T, Kadode M, Fujii M, Kawabata K, Abe T, Horiba M, Kohno S, and Nabe T (2015) Expression of CysLT2 receptors in asthma lung, and their possible role in bronchoconstriction. Allergol Int 64:351-358.

Sekioka T, Kadode M, Yonetomi Y, Kamiya A, Fujita M, Nabe T, and Kawabata K (2017) CysLT $_{2}$ receptor activation is involved in $\mathrm{LTC}_{4}$-induced lung air-trapping in guinea pigs. Eur $J$ Pharmacol 794:147-153.

Seow V, Lim J, Cotterell AJ, Yau MK, Xu W, Lohman RJ, Kok WM, Stoermer MJ, Sweet MJ, Reid RC, et al. (2016) Receptor residence time trumps drug-likeness and oral bioavailability in determining efficacy of complement C5a antagonists. Sci Rep 6:24575

Serra MF, Cotias AC, Pão CRR, Daleprane JB, Jurgilas PB, Couto GC, Anjos-Valotta EA, Cordeiro RSB, Carvalho VF, Silva PMR, et al. (2018) Repeated allergen exposure in $\mathrm{A} / \mathrm{J}$ mice causes steroid-insensitive asthma via a defect in glucocorticoid receptor bioavailability. J Immunol 201:851-860.

Sethi GS and Naura AS (2018) Progressive increase in allergen concentration abrogates immune tolerance in ovalbumin-induced murine model of chronic asthma. Int Immunopharmacol 60:121-131.

Sharma S, Muhammad F, and Chakraborty RK (2019) Asthma Medications, StatPearls Publishing, Treasure Island, FL. 
Shaw DE, Berry MA, Hargadon B, McKenna S, Shelley MJ, Green RH, Brightling CE, Wardlaw AJ, and Pavord ID (2007) Association between neutrophilic airway inflammation and airflow limitation in adults with asthma. Chest 132:1871-1875.

Shenoy SK, Drake MT, Nelson CD, Houtz DA, Xiao K, Madabushi S, Reiter E, Premont RT, Lichtarge O, and Lefkowitz RJ (2006) Beta-arrestin-dependent, G protein-independent ERK1/2 activation by the beta2 adrenergic receptor. $J$ Biol Chem 281:1261-1273.

Shenoy SK and Lefkowitz RJ (2005) Receptor-specific ubiquitination of beta-arrestin directs assembly and targeting of seven-transmembrane receptor signalosomes. $J$ Biol Chem 280:15315-15324.

Shiraishi Y, Asano K, Nakajima T, Oguma T, Suzuki Y, Shiomi T, Sayama K, Niimi K, Wakaki M, Kagyo J, et al. (2005) Prostaglandin D2-induced eosinophilic airway inflammation is mediated by CRTH2 receptor. J Pharmacol Exp Ther 312: 954-960.

Shiraishi Y, Asano K, Niimi K, Fukunaga K, Wakaki M, Kagyo J, Takihara T, Ueda S, Nakajima T, Oguma T, et al. (2008) Cyclooxygenase-2/prostaglandin D2/CRTH2 pathway mediates double-stranded RNA-induced enhancement of allergic airway inflammation. J Immunol 180:541-549.

Short PM, Williamson PA, Anderson WJ, and Lipworth BJ (2013) Randomized placebo-controlled trial to evaluate chronic dosing effects of propranolol in asthma. Am J Respir Crit Care Med 187:1308-1314.

Silva D and Jacinto T (2016) Inhaled $\beta_{2}$-agonists in asthma management: an evolving story. Breathe (Sheff) 12:375-377.

Simpson JL, Phipps S, Baines KJ, Oreo KM, Gunawardhana L, and Gibson PG (2014) Elevated expression of the NLRP3 inflammasome in neutrophilic asthma. Eur Respir J 43:1067-1076.

Singh D, Cadden P, Hunter M, Pearce Collins L, Perkins M, Pettipher R, Townsend $\mathrm{E}$, Vinall S, and O'Connor B (2013) Inhibition of the asthmatic allergen challenge response by the CRTH2 antagonist OC000459. Eur Respir J 41:46-52.

Singh D, Fuhr R, Jimenez L, Wählby Hamrén U, Jauhiainen A, Malice MP, Balaguer V, Lei A, Aggarwal A, Astbury C, et al. (2019) A randomized trial of dual-acting bronchodilator AZD8871 for chronic obstructive pulmonary disease. Am J Respir Crit Care Med 199:1282-1284.

Singh D, Pujol H, Ribera A, Seoane B, Massana E, Astbury C, Ruiz S, and de Miquel G (2014) A dose-ranging study of the bronchodilator effects of abeditero (LAS100977), a long-acting $\beta 2$-adrenergic agonist, in asthma; a Phase II, randomized study. BMC Pulm Med 14:176.

Singh S, Loke YK, and Furberg CD (2008) Inhaled anticholinergics and risk of major adverse cardiovascular events in patients with chronic obstructive pulmonary disease: a systematic review and meta-analysis. JAMA 300:1439-1450.

Siroux V and Garcia-Aymerich J (2011) The investigation of asthma phenotypes. Curr Opin Allergy Clin Immunol 11:393-399.

Slack RJ, Barrett VJ, Morrison VS, Sturton RG, Emmons AJ, Ford AJ, and Knowles RG (2013) In vitro pharmacological characterization of vilanterol, a novel longacting 32 -adrenoceptor agonist with 24 -hour duration of action. J Pharmacol Exp Ther 344:218-230.

Smirnov VM (1989) [Mechanisms of tachycardia caused by administration of atropine and vagotomy in animals of various species]. Kardiologiia 29:85-88.

Smith JS and Rajagopal S (2016) The $\beta$-arrestins: multifunctional regulators of G protein-coupled receptors. J Biol Chem 291:8969-8977.

Smith LJ, Shamsuddin M, and Houston M (1993) Effect of leukotriene D4 and platelet-activating factor on human alveolar macrophage eicosanoid and PAF synthesis. Am Rev Respir Dis 148:682-688.

Snetkov VA, Hapgood KJ, McVicker CG, Lee TH, and Ward JP (2001) Mechanisms of leukotriene D4-induced constriction in human small bronchioles. Br J Pharmacol 133:243-252.

Spada CS, Nieves AL, Krauss AH, and Woodward DF (1994) Comparison of leukotriene B4 and D4 effects on human eosinophil and neutrophil motility in vitro. J Leukoc Biol 55:183-191.

Spicuzza L, Belvisi MG, Birrell MA, Barnes PJ, Hele DJ, and Giembycz MA (2001) Evidence that the anti-spasmogenic effect of the beta-adrenoceptor agonist, isoprenaline, on guinea-pig trachealis is not mediated by cyclic AMP-dependent protein kinase. Br J Pharmacol 133:1201-1212.

Staus DP, Strachan RT, Manglik A, Pani B, Kahsai AW, Kim TH, Wingler LM, Ahn S, Chatterjee A, Masoudi A, et al. (2016) Allosteric nanobodies reveal the dynamic range and diverse mechanisms of G-protein-coupled receptor activation. Nature 535:448-452.

Steeland S, Vandenbroucke RE, and Libert C (2016) Nanobodies as therapeutics: big opportunities for small antibodies. Drug Discov Today 21:1076-1113.

Steinfeld T, Hughes AD, Klein U, Smith JA, and Mammen M (2011) THRX-198321 is a bifunctional muscarinic receptor antagonist and beta2-adrenoceptor agonist (MABA) that binds in a bimodal and multivalent manner. Mol Pharmacol $\mathbf{7 9}$ 389-399.

Stempel DA (2000) Montelukast added to inhaled beclomethasone in treatment of asthma. Am J Respir Crit Care Med 162:331-332.

Stockton JM, Birdsall NJ, Burgen AS, and Hulme EC (1983) Modification of the binding properties of muscarinic receptors by gallamine. Mol Pharmacol 23 $551-557$.

Struckmann N, Schwering S, Wiegand S, Gschnell A, Yamada M, Kummer W, Wess J, and Haberberger RV (2003) Role of muscarinic receptor subtypes in the constriction of peripheral airways: studies on receptor-deficient mice. Mol Pharmacol 64:1444-1451.

Sturton RG, Trifilieff A, Nicholson AG, and Barnes PJ (2008) Pharmacological characterization of indacaterol, a novel once daily inhaled 2 adrenoceptor agonist, on small airways in human and rat precision-cut lung slices. J Pharmacol Exp Ther 324:270-275

Sugimoto H, Shichijo M, Iino T, Manabe Y, Watanabe A, Shimazaki M, Gantner F, and Bacon KB (2003) An orally bioavailable small molecule antagonist of CRTH2, ramatroban (BAY u3405), inhibits prostaglandin D2-induced eosinophil migration in vitro. J Pharmacol Exp Ther 305:347-352.
Suguro H, Majima T, Hattori T, Ichimura K, Hashimoto N, and Horie T (2000) [Effect of a leukotriene antagonist, pranlukast hydrate, on exercise-induced asthma for 14 days]. Arerugi 49:646-652

Sun CX, Zhong H, Mohsenin A, Morschl E, Chunn JL, Molina JG, Belardinelli L, Zeng D, and Blackburn MR (2006) Role of A2B adenosine receptor signaling in adenosine-dependent pulmonary inflammation and injury. $J$ Clin Invest 116: 2173-2182.

Suno R, Lee S, Maeda S, Yasuda S, Yamashita K, Hirata K, Horita S, Tawaramoto MS, Tsujimoto H, Murata T, et al. (2018) Structural insights into the subtypeselective antagonist binding to the $\mathrm{M}_{2}$ muscarinic receptor. Nat Chem Biol 14 $1150-1158$

Svenningsen S and Nair P (2017) Asthma endotypes and an overview of targeted therapy for asthma. Front Med (Lausanne) 4:158.

Sykes DA, Bradley ME, Riddy DM, Willard E, Reilly J, Miah A, Bauer C, Watson SJ, Sandham DA, Dubois G, et al. (2016) Fevipiprant (QAW039), a slowly dissociating CRTh2 antagonist with the potential for improved clinical efficacy. Mol Pharmacol 89:593-605.

Szczuka A, Wennerberg M, Packeu A, and Vauquelin G (2009) Molecular mechanisms for the persistent bronchodilatory effect of the beta 2-adrenoceptor agonist salmeterol. Br J Pharmacol 158:183-194.

Takasaki J, Kamohara M, Matsumoto M, Saito T, Sugimoto T, Ohishi T, Ishii H, Ota T, Nishikawa T, Kawai Y, et al. (2000) The molecular characterization and tissue distribution of the human cysteinyl leukotriene CysLT(2) receptor. Biochem Biophys Res Commun 274:316-322.

Tanabe T, Fujimoto K, Yasuo M, Tsushima K, Yoshida K, Ise H, and Yamaya M (2008) Modulation of mucus production by interleukin-13 receptor alpha2 in the human airway epithelium. Clin Exp Allergy 38:122-134.

Tanaka K, Hirai H, Takano S, Nakamura M, and Nagata K (2004) Effects of prostaglandin D2 on helper $\mathrm{T}$ cell functions. Biochem Biophys Res Commun 316: $1009-1014$

Tautermann CS, Kiechle T, Seeliger D, Diehl S, Wex E, Banholzer R, Gantner F, Pieper MP, and Casarosa P (2013) Molecular basis for the long duration of action and kinetic selectivity of tiotropium for the muscarinic M3 receptor. $J$ Med Chem 56:8746-8756.

Terawaki K, Yokomizo T, Nagase T, Toda A, Taniguchi M, Hashizume K, Yagi T, and Shimizu T (2005) Absence of leukotriene B4 receptor 1 confers resistance to airway hyperresponsiveness and Th2-type immune responses. J Immunol 175: 4217-4225.

Thal DM, Sun B, Feng D, Nawaratne V, Leach K, Felder CC, Bures MG, Evans DA Weis WI, Bachhawat P, et al. (2016) Crystal structures of the M1 and M4 muscarinic acetylcholine receptors. Nature 531:335-340.

Thanawala VJ, Forkuo GS, Stallaert W, Leff P, Bouvier M, and Bond R (2014) Ligand bias prevents class equality among beta-blockers. Curr Opin Pharmacol 16:50-57.

Theron AJ, Steel HC, Tintinger GR, Gravett CM, Anderson R, and Feldman C (2014) Cysteinyl leukotriene receptor-1 antagonists as modulators of innate immune cell function. J Immunol Res 2014:608930.

Thivierge M, Doty M, Johnson J, Stanková J, and Rola-Pleszczynski M (2000) IL-5 up-regulates cysteinyl leukotriene 1 receptor expression in HL-60 cells differentiated into eosinophils. J Immunol 165:5221-5226.

Thomson NC, Chaudhuri R, and Livingston E (2004) Asthma and cigarette smoking. Eur Respir J 24:822-833.

Thorsen TS, Matt R, Weis WI, and Kobilka BK (2014) Modified T4 lysozyme fusion proteins facilitate G protein-coupled receptor crystallogenesis. Structure 22: 1657-1664.

Thurmond R, Chen B, Dunford P, Eckert W, Karlsson L, La D, Ward P, Xu X, and Greenspan A (2017) Pharmacology and clinical activity of toreforant, a histamine H4 receptor antagonist. Ann Pharmacol Pharm 2:1013.

Thurmond RL (2015) The histamine H4 receptor: from orphan to the clinic. Front Pharmacol 6:65.

Timmer W, Massana E, Jimenez E, Seoane B, de Miquel G, and Ruiz S (2014) First-in-human study of the safety, tolerability, pharmacokinetics and pharmacodynamics of abediterol (LAS100977), a novel long-acting B2-agonist. J Clin Pharmacol 54:1347-1353

Tintinger GR, Feldman C, Theron AJ, and Anderson R (2010) Montelukast: more than a cysteinyl leukotriene receptor antagonist? ScientificWorldJournal 10: 2403-2413.

Tohda Y, Nakahara H, Kubo H, Haraguchi R, Fukuoka M, and Nakajima S (1999) Effects of ONO-1078 (pranlukast) on cytokine production in peripheral blood mononuclear cells of patients with bronchial asthma. Clin Exp Allergy 29 $1532-1536$.

Tomankova T, Kriegova E, and Liu M (2015) Chemokine receptors and their therapeutic opportunities in diseased lung: far beyond leukocyte trafficking. Am J Physiol Lung Cell Mol Physiol 308:L603-L618.

Tomari S, Shimoda T, Kawano T, Mitsuta K, Obase Y, Fukushima C, Matsuse H, and Kohno S (2001) Effects of pranlukast, a cysteinyl leukotriene receptor 1 an tagonist, combined with inhaled beclomethasone in patients with moderate or severe asthma. Ann Allergy Asthma Immunol 87:156-161.

Torphy TJ (1994) Beta-adrenoceptors, cAMP and airway smooth muscle relaxation: challenges to the dogma. Trends Pharmacol Sci 15:370-374.

Trinh HKT, Suh DH, Nguyen TVT, Choi Y, Park HS, and Shin YS (2019) Characterization of cysteinyl leukotriene-related receptors and their interactions in a mouse model of asthma. Prostaglandins Leukot Essent Fatty Acids 141:17-23.

Turner CR, Breslow R, Conklyn MJ, Andresen CJ, Patterson DK, Lopez-Anaya A, Owens B, Lee P, Watson JW, and Showell HJ (1996) In vitro and in vivo effects of leukotriene B4 antagonism in a primate model of asthma. J Clin Invest $\mathbf{9 7}$ 381-387.

Turner CR, Smith WB, Andresen CJ, Swindell AC, and Watson JW (1994) Leukotriene D4 receptor antagonism reduces airway hyperresponsiveness in monkeys. Pulm Pharmacol 7:49-58. 
Uller L, Mathiesen JM, Alenmyr L, Korsgren M, Ulven T, Högberg T, Andersson G, Persson CG, and Kostenis E (2007) Antagonism of the prostaglandin D2 receptor CRTH2 attenuates asthma pathology in mouse eosinophilic airway inflammation. Respir Res 8:16.

Underwood DC, Osborn RR, Newsholme SJ, Torphy TJ, and Hay DW (1996) Persistent airway eosinophilia after leukotriene (LT) D4 administration in the guinea pig: modulation by the LTD4 receptor antagonist, pranlukast, or an interleukin-5 monoclonal antibody. Am J Respir Crit Care Med 154:850-857.

van der Westhuizen ET, Breton B, Christopoulos A, and Bouvier M (2014) Quantification of ligand bias for clinically relevant $\beta 2$-adrenergic receptor ligands: implications for drug taxonomy. Mol Pharmacol 85:492-509.

Van Heeke G, Allosery K, De Brabandere V, De Smedt T, Detalle L, and de Fougerolles A (2017) Nanobodies® as inhaled biotherapeutics for lung diseases. Pharmacol Ther 169:47-56.

Vaquerizo MJ, Casan P, Castillo J, Perpiña M, Sanchis J, Sobradillo V, Valencia A Verea H, Viejo JL, Villasante C, et al.; CASIOPEA (Capacidad de Singulair Oral en la Prevencion de Exacerbaciones Asmaticas) Study Group (2003) Effect of montelukast added to inhaled budesonide on control of mild to moderate asthma. Thorax 58:204-210.

Vargaftig BB and Singer M (2003) Leukotrienes mediate murine bronchopulmonary hyperreactivity, inflammation, and part of mucosal metaplasia and tissue injury induced by recombinant murine interleukin-13. Am J Respir Cell Mol Biol 28: 410-419.

Vauquelin G and Charlton SJ (2010) Long-lasting target binding and rebinding as mechanisms to prolong in vivo drug action. $B r J$ Pharmacol 161:488-508.

Vauquelin G and Charlton SJ (2013) Exploring avidity: understanding the potential gains in functional affinity and target residence time of bivalent and heterobivalent ligands. Br J Pharmacol 168:1771-1785.

Vauquelin G, Hall D, and Charlton SJ (2015) 'Partial' competition of heterobivalent ligand binding may be mistaken for allosteric interactions: a comparison of different target interaction models. Br J Pharmacol 172:2300-2315.

Violin JD, DiPilato LM, Yildirim N, Elston TC, Zhang J, and Lefkowitz RJ (2008) Beta2-adrenergic receptor signaling and desensitization elucidated by quantitative modeling of real time cAMP dynamics. $J$ Biol Chem 283: 2949-2961.

Virchow JC Jr, Prasse A, Naya I, Summerton L, and Harris A (2000) Zafirlukast improves asthma control in patients receiving high-dose inhaled corticosteroids. Am J Respir Crit Care Med 162:578-585.

Voehringer D, Reese TA, Huang X, Shinkai K, and Locksley RM (2006) Type 2 immunity is controlled by IL-4/IL-13 expression in hematopoietic non-eosinophil cells of the innate immune system. J Exp Med 203:1435-1446.

Wacker D, Fenalti G, Brown MA, Katritch V, Abagyan R, Cherezov V, and Stevens RC (2010) Conserved binding mode of human beta2 adrenergic receptor inverse agonists and antagonist revealed by X-ray crystallography. J Am Chem Soc 132: $11443-11445$.

Waldeck B (2002) Beta-adrenoceptor agonists and asthma--100 years of development. Eur J Pharmacol 445:1-12.

Walker JA, Barlow JL, and McKenzie AN (2013) Innate lymphoid cells--how did we miss them? Nat Rev Immunol 13:75-87.

Walker JK and DeFea KA (2014) Role for $\beta$-arrestin in mediating paradoxical $\beta 2 \mathrm{AR}$ and PAR2 signaling in asthma. Curr Opin Pharmacol 16:142-147.

Walker JK, Fong AM, Lawson BL, Savov JD, Patel DD, Schwartz DA, and Lefkowitz RJ (2003) Beta-arrestin-2 regulates the development of allergic asthma. J Clin Invest 112:566-574.

Walker JK, Penn RB, Hanania NA, Dickey BF, and Bond RA (2011) New perspectives regarding $\beta(2)$-adrenoceptor ligands in the treatment of asthma. $B r J$ Pharmacol 163:18-28.

Wallon C, Persborn M, Jönsson M, Wang A, Phan V, Lampinen M, Vicario M, Santos J, Sherman PM, Carlson M, et al. (2011) Eosinophils express muscarinic receptors and corticotropin-releasing factor to disrupt the mucosal barrier in ulcerative colitis. Gastroenterology 140:1597-1607.

Wan YY (2014) GATA3: a master of many trades in immune regulation. Trends Immunol 35:233-242.

Wang L, Yao D, Deepak R, Liu H, Xiao Q, Fan H, Gong W, Wei Z, and Zhang C (2018) Structures of the human PGD2 receptor CRTH2 reveal novel mechanisms for ligand recognition. $\mathrm{Mol}$ Cell 72:48-59.e4.

Wang XS and Lau HY (2006) Beta-adrenoceptor-mediated inhibition of mediator release from human peripheral blood-derived mast cells. Clin Exp Pharmacol Physiol 33:746-750.

Waseda K, Miyahara N, Kanehiro A, Ikeda G, Koga H, Fuchimoto Y, Kurimoto E, Tanimoto Y, Kataoka M, Tanimoto M, et al. (2011) Blocking the leukotriene B4 receptor 1 inhibits late-phase airway responses in established disease. Am J Respir Cell Mol Biol 45:851-857.

Watson N, Magnussen H, and Rabe KF (1997) Inherent tone of human bronchus: role of eicosanoids and the epithelium. Br J Pharmacol 121:1099-1104.

Weatherall M, Wijesinghe M, Perrin K, Harwood M, and Beasley R (2010) Metaanalysis of the risk of mortality with salmeterol and the effect of concomitant inhaled corticosteroid therapy. Thorax 65:39-43.

Weiß E and Kretschmer D (2018) Formyl-peptide receptors in infection, inflammation, and cancer. Trends Immunol 39:815-829.

Weichert D, Kruse AC, Manglik A, Hiller C, Zhang C, Hübner H, Kobilka BK, and Gmeiner P (2014) Covalent agonists for studying G protein-coupled receptor activation. Proc Natl Acad Sci USA 111:10744-10748.

Weiss JW, Drazen JM, Coles N, McFadden ER Jr, Weller PF, Corey EJ, Lewis RA, and Austen KF (1982a) Bronchoconstrictor effects of leukotriene C in humans. Science 216:196-198.

Weiss JW, Drazen JM, McFadden ER Jr, Weller PF, Corey EJ, Lewis RA, and Austen KF (1982b) Comparative bronchoconstrictor effects of histamine, leukotriene C, and leukotriene D in normal human volunteers. Trans Assoc Am Physicians 95:30-35.
Wenzel SE (2012) Asthma phenotypes: the evolution from clinical to molecular approaches. Nat Med 18:716-725.

Wenzel SE, Szefler SJ, Leung DY, Sloan SI, Rex MD, and Martin RJ (1997) Bronchoscopic evaluation of severe asthma: persistent inflammation associated with high dose glucocorticoids. Am J Respir Crit Care Med 156:737-743.

Wenzel SE, Westcott JY, and Larsen GL (1991) Bronchoalveolar lavage fluid mediator levels 5 minutes after allergen challenge in atopic subjects with asthma: relationship to the development of late asthmatic responses. J Allergy Clin Immunol 87:540-548.

White C, Wright A, and Brightling C (2018) Fevipiprant in the treatment of asthma. Expert Opin Investig Drugs 27:199-207.

Wills-Karp M, Luyimbazi J, Xu X, Schofield B, Neben TY, Karp CL, and Donaldson DD (1998) Interleukin-13: central mediator of allergic asthma. Science 282: 2258-2261.

Wisler JW, DeWire SM, Whalen EJ, Violin JD, Drake MT, Ahn S, Shenoy SK and Lefkowitz RJ (2007) A unique mechanism of beta-blocker action: carvedilo stimulates beta-arrestin signaling. Proc Natl Acad Sci USA 104:16657-16662.

Wood LG, Baines KJ, Fu J, Scott HA, and Gibson PG (2012) The neutrophilic inflammatory phenotype is associated with systemic inflammation in asthma. Chest 142:86-93.

Woodruff PG, Boushey HA, Dolganov GM, Barker CS, Yang YH, Donnelly S, Ellwanger A, Sidhu SS, Dao-Pick TP, Pantoja C, et al. (2007) Genome-wide profiling identifies epithelial cell genes associated with asthma and with treatment response to corticosteroids. Proc Natl Acad Sci USA 104:15858-15863.

Woodruff PG, Modrek B, Choy DF, Jia G, Abbas AR, Ellwanger A, Koth LL, Arron JR, and Fahy JV (2009) T-helper type 2-driven inflammation defines major subphenotypes of asthma. Am J Respir Crit Care Med 180:388-395.

Woodward DF, Jones RL, and Narumiya S (2011) International Union of Basic and Clinical Pharmacology. LXXXIII: classification of prostanoid receptors, updating 15 years of progress. Pharmacol Rev 63:471-538.

Wu W, Bleecker E, Moore W, Busse WW, Castro M, Chung KF, Calhoun WJ, Erzurum S, Gaston B, Israel E, et al. (2014) Unsupervised phenotyping of Severe Asthma Research Program participants using expanded lung data. J Allergy Clin Immunol 133:1280-1288.

Wynn TA (2015) Type 2 cytokines: mechanisms and therapeutic strategies. Nat Rev Immunol 15:271-282.

Xue L, Barrow A, Fleming VM, Hunter MG, Ogg G, Klenerman P, and Pettipher R (2012) Leukotriene E4 activates human Th2 cells for exaggerated proinflammatory cytokine production in response to prostaglandin D2. J Immunol 188:694-702.

Xue L, Barrow A, and Pettipher R (2009) Novel function of CRTH2 in preventing apoptosis of human Th2 cells through activation of the phosphatidylinositol 3kinase pathway. J Immunol 182:7580-7586.

Xue L, Gyles SL, Wettey FR, Gazi L, Townsend E, Hunter MG, and Pettipher R (2005) Prostaglandin D2 causes preferential induction of proinflammatory Th2 cytokine production through an action on chemoattractant receptor-like molecule expressed on Th2 cells. J Immunol 175:6531-6536.

Xue L, Salimi M, Panse I, Mjösberg JM, McKenzie AN, Spits H, Klenerman P, and Ogg G (2014) Prostaglandin D2 activates group 2 innate lymphoid cells through chemoattractant receptor-homologous molecule expressed on TH2 cells. J Allergy Clin Immunol 133:1184-1194.

Yamaguchi T, Kohrogi H, Honda I, Kawano O, Sugimoto M, Araki S, and Ando M (1992) A novel leukotriene antagonist, ONO-1078, inhibits and reverses human bronchial contraction induced by leukotrienes $\mathrm{C} 4$ and D4 and antigen in vitro. Am Rev Respir Dis 146:923-929.

Yamaoka KA and Kolb JP (1993) Leukotriene B4 induces interleukin 5 generation from human T lymphocytes. Eur J Immunol 23:2392-2398.

Yamauchi K, Tanifuji Y, Pan LH, Yoshida T, Sakurai S, Goto S, Kuroda S, Kobayash $\mathrm{H}$, and Inoue H (2001) Effects of pranlukast, a leukotriene receptor antagonist, on airway inflammation in mild asthmatics. J Asthma 38:51-57.

Yan D, Hamed O, Joshi T, Mostafa MM, Jamieson KC, Joshi R, Newton R, and Giembycz MA (2018) Analysis of the indacaterol-regulated transcriptome in human airway epithelial cells implicates gene expression changes in the adverse and therapeutic effects of $\beta_{2}$-adrenoceptor agonists. J Pharmacol Exp Ther $\mathbf{3 6 6}$ : 220-236.

Yan H, Deshpande DA, Misior AM, Miles MC, Saxena H, Riemer EC, Pascual RM, Panettieri RA, and Penn RB (2011) Anti-mitogenic effects of $\beta$-agonists and PGE2 on airway smooth muscle are PKA dependent. FASEB J 25:389-397.

Yanagawa H, Sugita A, Azuma M, Ogawa H, Kitamuro C, Yoneda K, Shinkawa K Tani K, and Sone S (2004) Long-term follow-up of pulmonary function in bronchial asthma patients treated with pranlukast. Hai 182:51-58.

Yarova PL, Stewart AL, Sathish V, Britt RD Jr, Thompson MA, P Lowe AP, Freeman M, Aravamudan B, Kita H, Brennan SC, et al. (2015) Calcium-sensing receptor antagonists abrogate airway hyperresponsiveness and inflammation in allergic asthma. Sci Transl Med 7:284ra60.

Yasui H, Fujisawa T, Inui N, Kato M, Hashimoto D, Enomoto N, Nakamura Y, Shirai T, Suda T, Nakamura H, et al. (2012) Impact of add-on pranlukast in stable asthma; the additive effect on peripheral airway inflammation. Respir Med 106 508-514.

Yazid S, Solito E, Christian H, McArthur S, Goulding N, and Flower R (2009) Cromoglycate drugs suppress eicosanoid generation in U937 cells by promoting the release of Anx-A1. Biochem Pharmacol 77:1814-1826.

Yokomizo T, Nakamura M, and Shimizu T (2018) Leukotriene receptors as potential therapeutic targets. J Clin Invest 128:2691-2701.

Yokoyama A, Kohno N, Sakai K, Hirasawa Y, Kondo K, and Hiwada K (1998) Effect of pranlukast, a leukotriene receptor antagonist, in patients with severe asthma refractory to corticosteroids. J Asthma 35:57-62.

Yonetomi Y, Sekioka T, Kadode M, Kitamine T, Kamiya A, Matsumura N, Fujita M, and Kawabata K (2015) Leukotriene C4 induces bronchoconstriction and airway vascular hyperpermeability via the cysteinyl leukotriene receptor 2 in S-hexyl glutathione-treated guinea pigs. Eur J Pharmacol 754:98-104. 
Yoo SH, Park SH, Song JS, Kang KH, Park CS, Yoo JH, Choi BW, and Hahn MH; Representing Korea Pranlukast Study Group (2001) Clinical effects of pranlukast, an oral leukotriene receptor antagonist, in mild-to-moderate asthma: a 4 week randomized multicentre controlled trial. Respirology 6:15-21.

Yoshida S, Ishizaki Y, Shoji T, Onuma K, Nakagawa H, Nakabayashi M, Akahori K, Hasegawa H, and Amayasu H (2000a) Effect of pranlukast on bronchial inflammation in patients with asthma. Clin Exp Allergy 30:1008-1014.

Yoshida S, Sakamoto H, Ishizaki Y, Onuma K, Shoji T, Nakagawa H, Hasegawa H, Nakabayashi M, and Amayasu H (2000b) Efficacy of leukotriene receptor antagonist in bronchial hyperresponsiveness and hypersensitivity to analgesic in aspirinintolerant asthma. Clin Exp Allergy 30:64-70.

Yu X, Marshall MJE, Cragg MS, and Crispin M (2017) Improving antibody-based cancer therapeutics through glycan engineering. BioDrugs 31:151-166.

Yukawa T, Ukena D, Kroegel C, Chanez P, Dent G, Chung KF, and Barnes PJ (1990) Beta 2-adrenergic receptors on eosinophils. Binding and functional studies. Am Rev Respir Dis 141:1446-1452.

Zhang C, Srinivasan Y, Arlow DH, Fung JJ, Palmer D, Zheng Y, Green HF, Pandey A, Dror RO, Shaw DE, et al. (2012) High-resolution crystal structure of human protease-activated receptor 1. Nature 492:387-392.

Zhang D, Gao ZG, Zhang K, Kiselev E, Crane S, Wang J, Paoletta S, Yi C, Ma L, Zhang W, et al. (2015) Two disparate ligand-binding sites in the human P2Y1 receptor. Nature 520:317-321.

Zhang DH, Yang L, Cohn L, Parkyn L, Homer R, Ray P, and Ray A (1999) Inhibition of allergic inflammation in a murine model of asthma by expression of a dominantnegative mutant of GATA-3. Immunity 11:473-482.
Zhang J, Zhang K, Gao ZG, Paoletta S, Zhang D, Han GW, Li T, Ma L, Zhang W, Müller CE, et al. (2014a) Agonist-bound structure of the human P2Y12 receptor. Nature 509:119-122

Zhang K, Zhang J, Gao ZG, Zhang D, Zhu L, Han GW, Moss SM, Paoletta S, Kiselev $\mathrm{E}, \mathrm{Lu} \mathrm{W}$, et al. (2014b) Structure of the human P2Y12 receptor in complex with an antithrombotic drug. Nature 509:115-118.

Zheng W and Flavell RA (1997) The transcription factor GATA-3 is necessary and sufficient for Th2 cytokine gene expression in CD4 T cells. Cell 89:587-596.

Zhong H, Belardinelli L, Maa T, Feoktistov I, Biaggioni I, and Zeng D (2004) A(2B) adenosine receptors increase cytokine release by bronchial smooth muscle cells. Am J Respir Cell Mol Biol 30:118-125.

Zhong H, Belardinelli L, Maa T, and Zeng D (2005) Synergy between A2B adenosine receptors and hypoxia in activating human lung fibroblasts. Am J Respir Cell Mol Biol 32:2-8.

Zhu BT (1996) Rational design of receptor partial agonists and possible mechanisms of receptor partial activation: a theory. J Theor Biol 181:273-291.

Zhu L, Ciaccio CE, and Casale TB (2018) Potential new targets for drug development in severe asthma. World Allergy Organ J 11:30.

Zieba BJ, Artamonov MV, Jin L, Momotani K, Ho R, Franke AS, Neppl RL, Stevenson AS, Khromov AS, Chrzanowska-Wodnicka M, et al. (2011) The cAMPresponsive Rap1 guanine nucleotide exchange factor, Epac, induces smooth muscle relaxation by down-regulation of RhoA activity. J Biol Chem 286:16681-16692.

Zosky GR and Sly PD (2007) Animal models of asthma. Clin Exp Allergy 37:973-988.

Zou Y, Weis WI, and Kobilka BK (2012) N-terminal T4 lysozyme fusion facilitates crystallization of a G protein coupled receptor. PLoS One 7:e46039. 